\title{
Nanotechnology for single crystalline silicon solar cell applications
}

\author{
by \\ Shuyu Ding, B. Eng \\ A thesis submitted to the \\ Faculty of Graduate and Postdoctoral Affairs \\ in partial fulfillment of the requirements for the degree of
}

Master of Applied Science in Electrical and Computer Engineering

Ottawa-Carleton Institute for Electrical and Computer Engineering Department of Electronics

Carleton University

Ottawa, Ontario

May, 2015

(c) Copyright,

Shuyu Ding, 2015 
The undersigned hereby recommends to the

Faculty of Graduate and Postdoctoral Affairs

acceptance of the thesis

Nanotechnology for single crystalline silicon solar cell applications

Submitted by Shuyu Ding, B. Eng

in partial fulfillment of the requirements for the degree of

Master of Applied Science in Electrical and Computer Engineering

Professor Winnie N. Ye, Thesis Supervisor

Professor Niall Tait, Chair, Department of Electrical and Computer Engineering

Ottawa-Carleton Institute for Electrical and Computer Engineering

Department of Electronics

Carleton University

May, 2015 


\section{Abstract}

Solar cells are receiving a lot of attention due to the ongoing climate debate and attempts to implement more green energy sources to reduce the $\mathrm{CO}_{2}$ emissions in the atmosphere. Single crystalline silicon (c-Si) solar cells with better stability over a long time period compared to other silicon based solar cells have taken a main position in the solar cell market. As new solar cell technology continually develops, the disadvantage of the relatively high cost of electricity generated related to low cell efficiency becomes one of top issues with c-Si solar cells. The purpose of this thesis is to investigate the effectiveness of nanotechnologies, in particular plasmonic metal nanoparticles (MNPs), black silicon (b-Si) nanostructures and horizontally-grown silicon nanowires (SiNWs) structures, for cell efficiency enhancement of c-Si solar cells through numerical simulation and experimental demonstrations. Due to the advantage of c-Si solar cells on the material cost, the nanotechnologies enable lower cost compared to the other types solar cell technologies with the same efficiency enhancement.

We first investigate the optical and the electrical properties of MNPs for c-Si solar cell applications based on the finite difference time domain method. Simulations are performed to optimize the MNPs in terms of shape, size, surface coverage, and dielectric environment. Silver nanocubes with a silicon dioxide $\left(\mathrm{SiO}_{2}\right)$ sublayer are experimentally demonstrated to obtain up to an average of $7 \%$ cell efficiency enhancement. To the best of our knowledge, our group was the first group that utilizes the cubic silver MNPs on the c-Si solar cells with experimental demonstrations.

Next, we investigate the cell efficiency enhancement due to the reduction of surface reflection from nanostructures. Two techniques are discussed, (i) the growth of black 
silicon and (ii) the horizontally-grown SiNWs structures. The b-Si structures are first optimized in terms of shape, size, and aspect ratio, and their effect on surface reflectivity is characterized. The needle-shaped b-Si structures are shown in experiment to obtain near-zero specular reflectivity and average $1.9 \%$ diffused reflectivity. Similarly, the horizontally-grown SiNWs structures grown by the vapor-liquid-solid (VLS) process with a 4-hour growing time have shown to reduce reflectivity from $45 \%$ to $25 \%$ at 400 $\mathrm{nm}$ wavelengths. The fabrication procedure and experimental measurement results of SiNWs structure on c-Si substrates are explained in detail. 


\section{Acknowledgements}

I would like to acknowledge the help and contributions of all the people who made this thesis possible, without you I would never have made it to this point. First I would like to acknowledge my supervisor, Dr. Winnie N. Ye, who was a constant source of guidance and motivation throughout this degree. My fellow students Yule Xiong, Ryan Veenkamp, Owen Marsh, Dusan Gostimirovic and Yao Sun provided me with invaluable advice and friendship, which I will always be grateful for. I want to thank the Carleton Microfabrication Lab technicians Rob Vandusen, Angela McCormick and Rick Adams for their invaluable assistance and input in fabricating the solar cell as well as Dr. Garry Tarr for helping in designing and fabricating the solar cell sublayer and the black silicon nanostructures. I would like to thank my collaborator Adam Bottomley at the Department of Chemistry who synthesizing and depositing the metal nanoparticles on solar cell which made the experiment possible. Testing would not have been possible without Ross Cheriton at SUNLAB, thank you for your help and input. I acknowledge the warmth and support from the students and staff in the Department of Electronics at Carleton University, who made my stay here so enjoyable. Last but not the least, I would like to thank my family: my parents Qin Ding and Lei Wu, for giving birth to me in the first place and supporting me spiritually throughout my life. 


\section{Table of Contents}

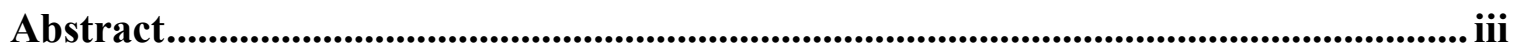

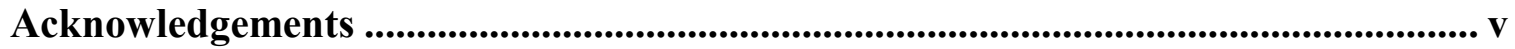

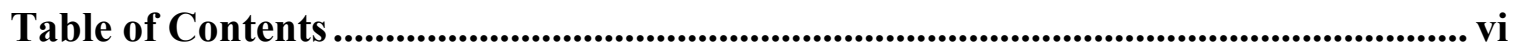

List of Illustrations................................................................................................................... ix

List of Appendices...................................................................................................................... Xv

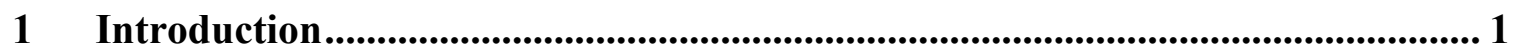

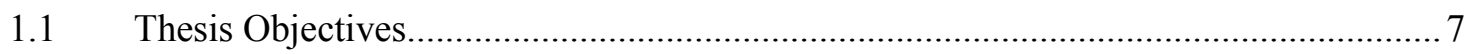

2 Literature Review and Background............................................................... 10

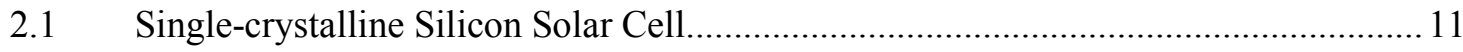

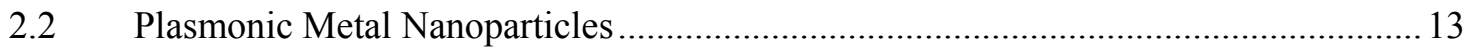

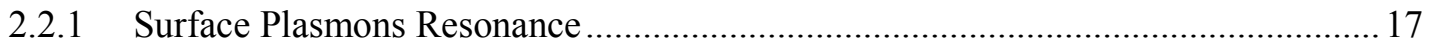

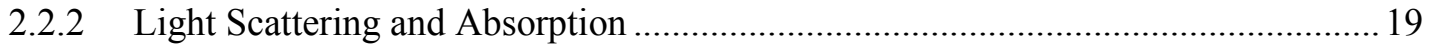

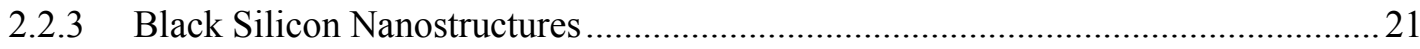

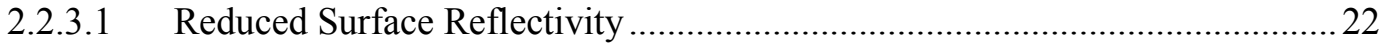

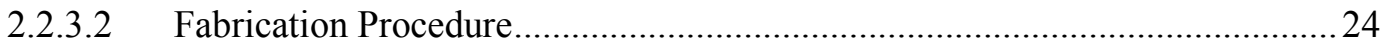

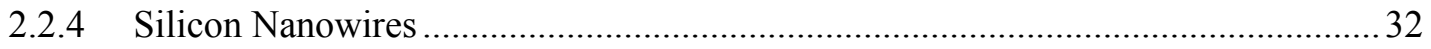

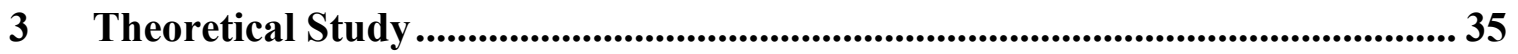

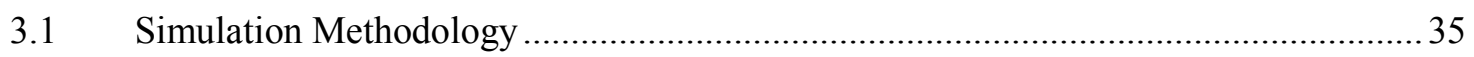

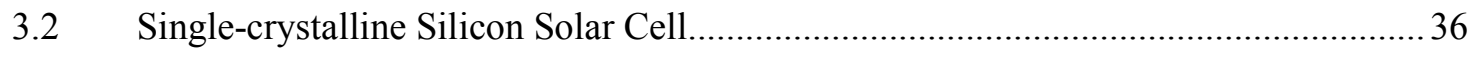

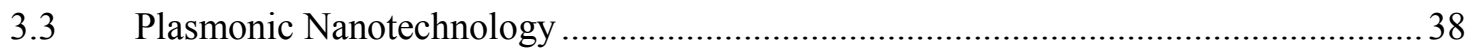

3.3.1 Effect of Ag MNPs Shape on Plasmonic and Efficiency Enhancement ................. 40

3.3.2 Effect of Ag MNPs Size on Efficiency Enhancement .......................................... 44

3.3.3 Effect of Ag MNPs Surrounding Media on Plasmonics and Efficiency Enhancement 46 
3.3.4 Effect of Ag MNPs Surface Coverage on Solar Cell efficiency ..............................52

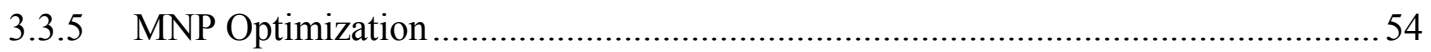

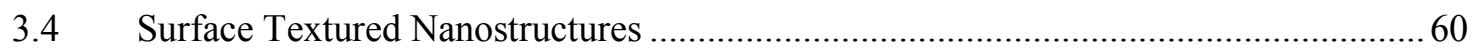

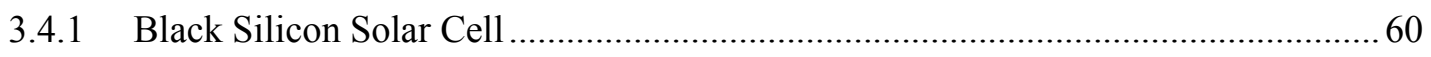

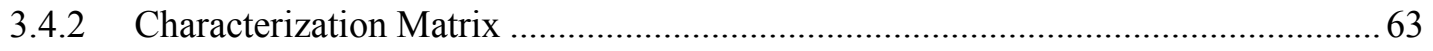

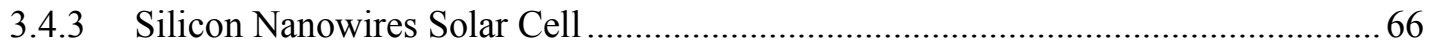

$4 \quad$ Experimental Design .................................................................................................... 69

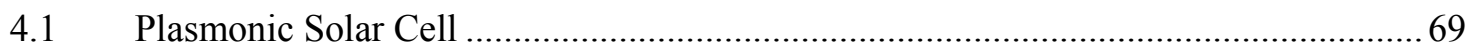

4.1.1 Silver Metal Nanoparticles (MNPs) Fabrication Method ....................................... 70

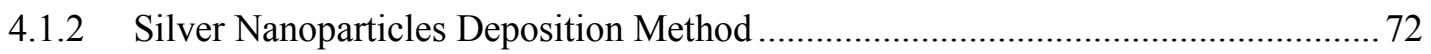

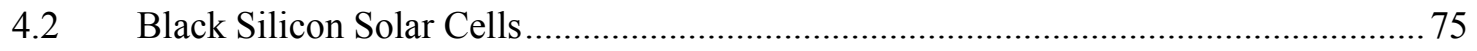

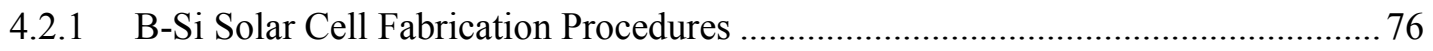

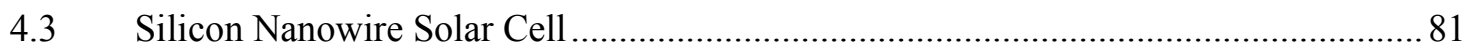

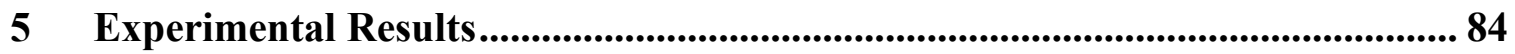

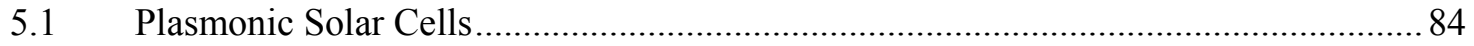

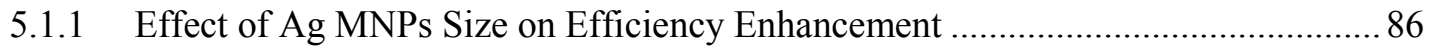

5.1.2 Effect of Sublayer Material on Efficiency Enhancement.......................................91

5.1.3 Effect of Ag MNPs on Surface Reflectivity ........................................................... 93

5.1.4 Silver Oxide Effect on Efficiency Enhancement …............................................ 95

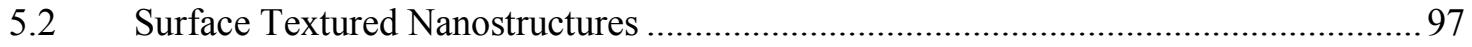

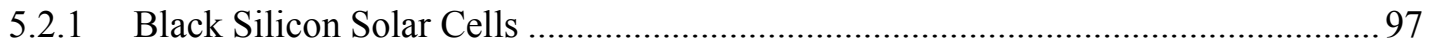

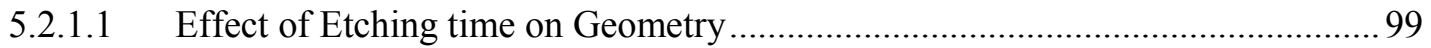

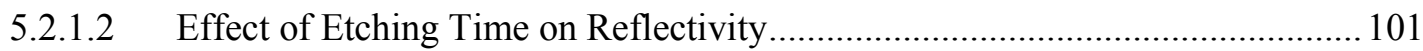

5.2.1.3 Effect of Fabrication Process on b-Si Structures ............................................... 104

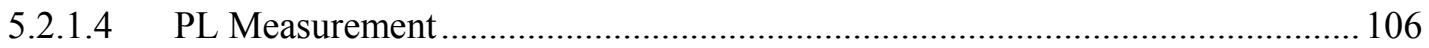

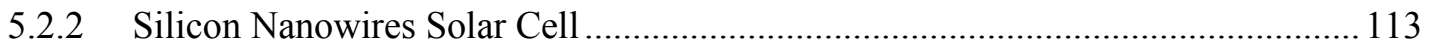


5.3 Experimental Results Summary

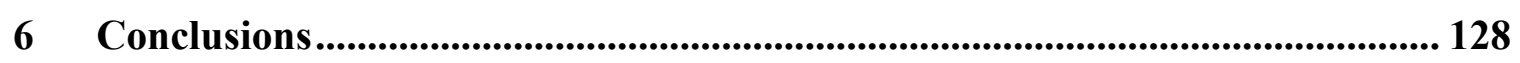

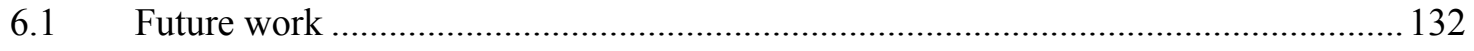

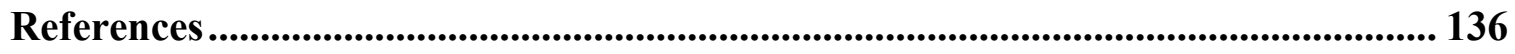

Appendix ....................................................................................................................... 142 


\section{List of Illustrations}

Figure 1-1: World primal energy consumption grew from 1988 to 2013. (Source: BP statistical review of world energy 2014[1]) ………............................................... 1

Figure 1-2: Renewable energy share of global electricity production in 2013 [2] ............ 2

Figure 1-3: Solar PV module share by global development, END 2013[7] ...................... 3

Figure 2-1: The SPR polariton propagating at the interface between a dielectric and a

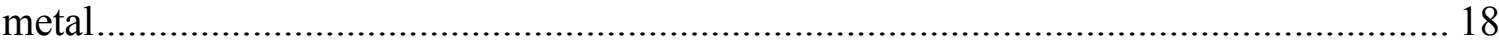

Figure 2-2: The LSPR polariton propagating at the nanoparticle surface ……………... 18

Figure 2-3: Geometry for incident wave scattering by particle of arbitrary shape ........... 19

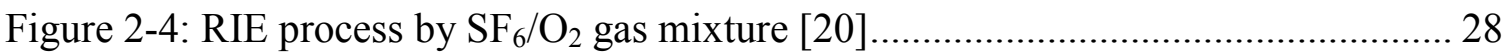

Figure 2-5: (a) Photo image of the ECR etching system in CUMFF (b) The construction

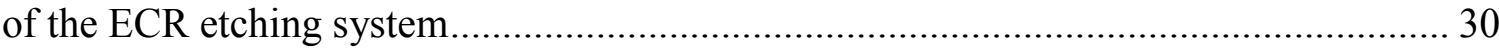

Figure 3-1: (a) A two-dimensional schematic view of a simulated p-n junction c-Si solar

cell, (b) Simulation result for the c-Si solar cell build in the simulator........................... 36

Figure 3-2: Electric field distribution of Ag MNP deposited on c-Si solar cell (a) cube with $80 \mathrm{~nm}$ in edge length and (b) hemisphere with $80 \mathrm{~nm}$ in diameter ........................ 41

Figure 3-3: Schematic view of c-Si solar cell with (a) $80 \mathrm{~nm}$ size cubic Ag MNPs, and (b) $80 \mathrm{~nm}$ size hemispherical Ag MNPs. Simulation results for solar cell with (c) cubic Ag MNPs and (d) hemispherical Ag MNPs ...................................................................... 43

Figure 3-4: Cubic Ag MNPs on c-Si solar cell with different edge length....................... 44

Figure 3-5: Electric field distribution of $\mathrm{Ag} \mathrm{MNP}$ set on $10 \mathrm{~nm}$ thick $\mathrm{SiO}_{2}$ sublayer (a) 80

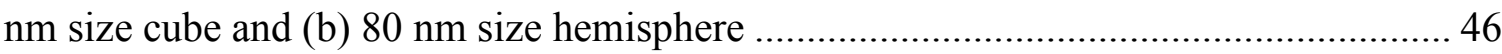


Figure 3-6: Schematic view of $10 \mathrm{~nm}$ thick $\mathrm{SiO}_{2}$ sublayer on c-Si solar cell with (a) 80 nm size cubic Ag MNPs, and (b) $80 \mathrm{~nm}$ size hemispherical Ag MNPs. Simulation results for solar cell with (c) cubic Ag MNPs and (d) hemispherical Ag MNPs 49 Figure 3-7: Efficiency enhancement by $80 \mathrm{~nm}$ size cubic Ag MNPs and hemispherical $\mathrm{Ag}$ MNPs with 20\% surface coverage on different thickness $\mathrm{SiO}_{2}$ sublayer 50

Figure 3-8: $80 \mathrm{~nm}$ size cubic Ag MNPs and $80 \mathrm{~nm}$ size hemispherical Ag MNPs deposited on the c-Si solar cell with different surface coverages 52 Figure 3-9: Simulated geometry for c-Si solar cells with Al back contact, c-Si absorber, and $\mathrm{SiO}_{2}$ sublayer for (a) hemispherical Ag MNPs and (b) cubic Ag MNPs 54

Figure 3-10: Optimized efficiency enhancement of 80nm size hemispherical Ag MNPs with different surface coverages and $\mathrm{SiO}_{2}$ sublayer thicknesses . 56 Figure 3-11: Optimized efficiency enhancement of $80 \mathrm{~nm}$ size cubic Ag MNPs with different surface coverages and $\mathrm{SiO}_{2}$ sublayer thicknesses 58

Figure 3-12: Needle-like shape, pyramid shape, and cylinder shape silicon structures ... 60 Figure 4-1: Illustration of the reaction path leading to well-defined cubic Ag MNP [42].

Figure 4-2: (a) SEM image of cubic Ag MNPs with $90 \mathrm{~nm}$ in edge length stuck together in the reaction solution after the reaction [40]and (b) photo of a flask containing cubic $\mathrm{Ag}$ MNPs with $90 \mathrm{~nm}$ in edge length stored in the cubic Ag MNPs solution after the reaction $[40]$ 72

Figure 4-3: Photo of Langmuir-Blodgett (LB) monolayer trough used to prepare cubic Ag MNPs monolayer and deposit the monolayer on solar cells. 72 
Figure 4-4: AFM topography images of (a) cubic Ag MNPs in the MNPs solution after the synthesis reaction and (b) substrates of cubic Ag MNPs mixed with phospholipid molecules mixture liquid [41] 73

Figure 4-5: AFM topography images of cubic Ag MNPs mixed with phospholipids mixtures deposited on substrate at different surface pressure, (a) $0.1 \mathrm{mN} / \mathrm{m}$, (b) $15 \mathrm{mN} / \mathrm{m}$, (c) $35 \mathrm{mN} / \mathrm{m}[41]$ 75

Figure 4-6: Fabrication procedures for c-Si solar cells with ECR texturing 77

Figure 5-1: AFM image of cubic Ag MNPs with $40 \mathrm{~nm}$ in edge length deposited on a pure c-Si solar cell within $5 \mu \mathrm{m} \times 5 \mu \mathrm{m}$ 85

Figure 5-2: Experimental results from c-Si solar cells with and without $40 \mathrm{~nm}$ size cubic Ag MNPs 86

Figure 5-3: AFM image of cubic Ag MNPs with $75 \mathrm{~nm}$ in edge length deposited on the cSi solar cell. 88

Figure 5-4: Experimental results of the c-Si solar cell with and without $75 \mathrm{~nm}$ size cubic Ag MNPs 88

Figure 5-5: AFM image of $80 \mathrm{~nm}$ size cubic Ag MNPs deposited on the c-Si solar cell. 90 Figure 5-6: Experimental results for the solar cells with $80 \mathrm{~nm}$ size cubic Ag MNPs..... 90 Figure 5-7: Experimental results for the c-Si solar cells with $80 \mathrm{~nm}$ size cubic Ag MNPs deposited on a $25 \mathrm{~nm}$ thickness $\mathrm{SiO}_{2}$ sublayer 91 Figure 5-8: Experimental results for the c-Si solar cells with $80 \mathrm{~nm}$ size cubic Ag MNPs deposited on a $35 \mathrm{~nm}$ thickness $\mathrm{SiO}_{2}$ sublayer 91 Figure 5-9: Reflection spectroscopy of the c-Si solar cell with 75nm size cubic Ag MNPs deposited on the front surface 93 
Figure 5-10: Reflection spectroscopy of the c-Si wafer and the $\mathrm{SiO}_{2}$ sublayer with and without $80 \mathrm{~nm}$ size cubic Ag MNPs

Figure 5-11: Experimental results measured at different days for c-Si solar cells with 80

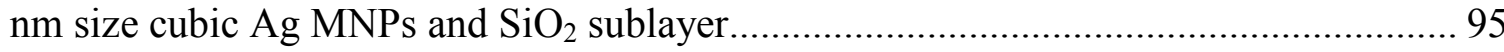

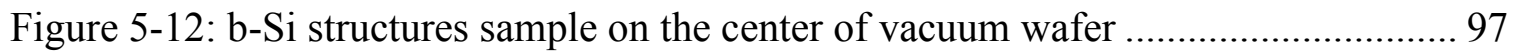

Figure 5-13: SEM images of b-Si structures etched after 50 minutes from (a) $45^{\circ}$ top

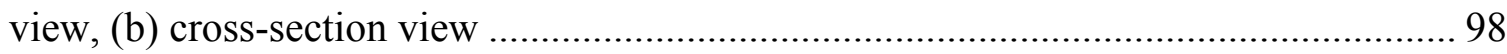

Figure 5-14: SEM cross-section views of b-Si structures etched after (a) 45 minutes and

(b) $50 \mathrm{~min}$ 99

Figure 5-15: SEM images of needle-like shaped structures width characterization for b-Si solar cells with (a) 45 minutes etching time and (b) 50 minutes etching time 100 Figure 5-16: Specular reflectivity of b-Si wafer sample etched after $50 \mathrm{~min}$ 101 Figure 5-17: Light intensity of the incident light on the b-Si sample and the reflected light from a b-Si sample with 50 minutes in etching time 102 Figure 5-18: Diffused reflectivity measurement for b-Si solar cells with different etching times. 103

Figure 5-19: SEM images for b-Si nanostructures after (a) photoresist layer deposition and (b) standard RCA cleaning process. 105 Figure 5-20: Photo image of the PL measurement system in the SUNLAB 106 Figure 5-21: PL measurements obtained from b-Si samples formed by ECR at different etching times 107 Figure 5-22: PL measurements obtained from b-Si sample with a 45 minute etching time at different excitation power levels 109 
Figure 5-23: PL measurements obtained from b-Si sample with a 45 minute etching time at different temperatures 110

Figure 5-24: PL measurements obtained from b-Si samples etched for 45 minutes with and without RTA process

Figure 5-25: SEM top view image of c-Si solar cell with SiNW growth after 1 hour ... 114 Figure 5-26: SEM images of SiNWs solar cell after growth time: (a) 0.5 hours (Sample \#15), (b) 1 hour (Sample \#4), (c) 1 hour (Sample \#8) and (d) 2 hours (Sample \#9) ...... 115 Figure 5-27: Efficiency measurements of SiNWs solar cell with different growth time 116 Figure 5-28: SEM images of SiNWs with irregular-shapes on c-Si solar cell after 4 hours of growth time: (a) $1.71 \mu \mathrm{m}$ (Sample \#12), (b) $1.75 \mu \mathrm{m}$ (Sample \#1) and (c) $2.55 \mu \mathrm{m}$ (Sample \#2) 117 Figure 5-29: Efficiency measurements of c-Si solar cell with irregular-shaped SiNWs 117 Figure 5-30: SEM images of SiNWs on c-Si solar cell after 1 hour growth time: (a) 0.38 $\mu \mathrm{m}$ (Sample \#17), (b) $1.02 \mu \mathrm{m}$ (Sample \#14), (c) $6.27 \mu \mathrm{m}$ (Sample \#16)..... 119 Figure 5-31: Efficiency measurements of c-Si solar cell with wire shaped SiNWs after 1 hour growth time 120 Figure 5-32: SEM images of SiNWs on c-Si solar cell after 2 hours growth time: (a) 2.1 $\mu \mathrm{m}$ (Sample \#3), (b) $2.5 \mu \mathrm{m}$ (Sample \#13), (c) $6.02 \mu \mathrm{m}$ (Sample \#11) 121 Figure 5-33: Efficiency measurements of c-Si solar cell with wire-shaped SiNWs after 2 hours growth time 121

Figure 5-34: Total reflectance measurement of c-Si solar cell with and without SiNWs 122 
Figure 5-35: Efficiency measurements of c-Si solar cell with different growth times of SiNWs... 124

Figure 6-1: SEM topography image of cubic Ag MNPs deposited on c-Si solar cell ... 133 


\section{List of Appendices}

Appendix A: The Mie Theory........................................... 142

Appendix B: Fabrication Process Runsheet for single crystalline silicon (c-Si) solar

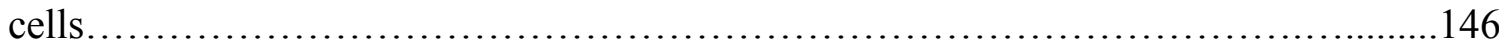

Appendix C: Fabrication Process Runsheet for black silicon (b-Si) solar cells

Appendix D: Fabrication Process Runsheet for silicon nanowires (SiNWs) solar cells 


\section{Introduction}

In 2013, world primary energy consumption was 12,730 mega tons (Mtoe) of oil equivalent (Mtoe) [1], among which, $87.8 \%$ of the power consumed was provided by fossil fuels and other non-renewable fuels, and only $2.2 \%$ was provided by renewable energy like wind and solar. Fig. 1-1 shows the world primary energy consumption in 2013 and the world energy consumption growth from 1988 to 2013 . The rate of coal energy consumption grew $3.8 \%$ in 2013 , an increment of 100 Mtoe. The rate of renewable energy consumption increased by $20 \%$ in 2013 , corresponding to a 12.5 Mtoe increment. It is believed that renewable energy had the largest growth rate with the lowest consumption increment among all energy resources. In addition, renewable energy as a combination of solar photovoltaic (PV), wind, among others, has become an interesting topic around the world since the combustion of fossil fuels creates greenhouse gas emissions, which has been confirmed as the main reason for global warming. Thus, developing and enhancing renewable energy sources to replace fossil fuels consumption are needed as soon as possible.

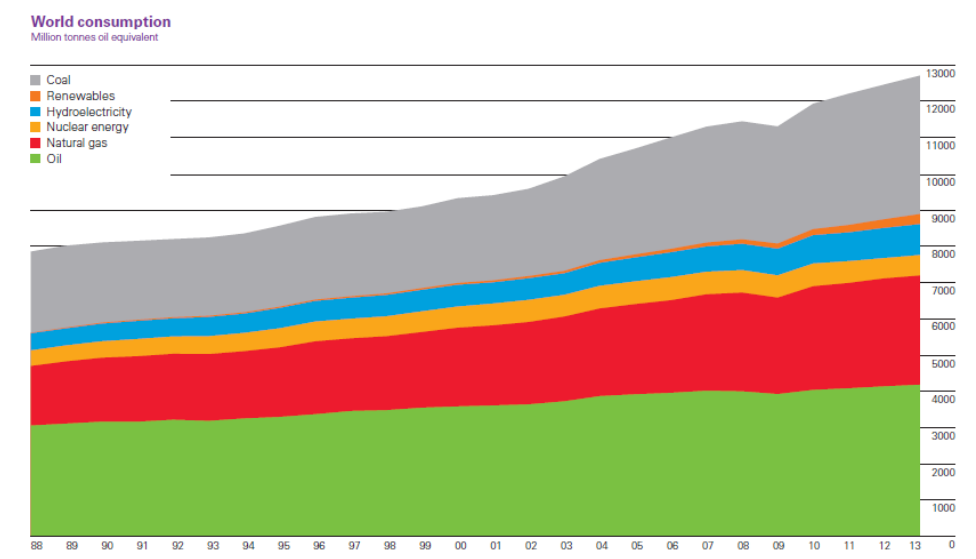

Figure 1-1: World primal energy consumption grew from 1988 to 2013. (Source: BP statistical review of world energy 2014[1]) 
Using a harmful, scarce resource to stratify the energy demands of an increasing population has gained global attention, prompting an increase in research opportunities in the field of renewable energy. Renewable energy involves various energy resources, among which are hydro, wind, and solar. In 2013, more than half of renewable energy in the global electricity generation market was produced by hydro energy, followed by wind energy and solar PV energy supplying $2.9 \%$ and $0.7 \%$, respectively [2]. Fig. 1-2 shows the percentage of renewable energy electricity production in global electricity production in 2013. Even though solar energy is not at the top of the list, it is starting to play a substantial role in the electricity generation market in countries, such as Germany and Italy [3]. Presently, solar PV energy is considered to be the cleanest and most abundant energy source on earth. Being able to harness this energy as efficiently as possible would contribute to decreasing greenhouse gas emissions and reducing the risks of climate change. The annual growth rate of solar PV energy capacity was $39 \%$ in 2013 , which was the highest growth rate among all renewable energy resources [2]. It is anticipated that the capacity of solar PV will continue to expand. This thesis focuses on solar PV technology based on silicon solar cells.

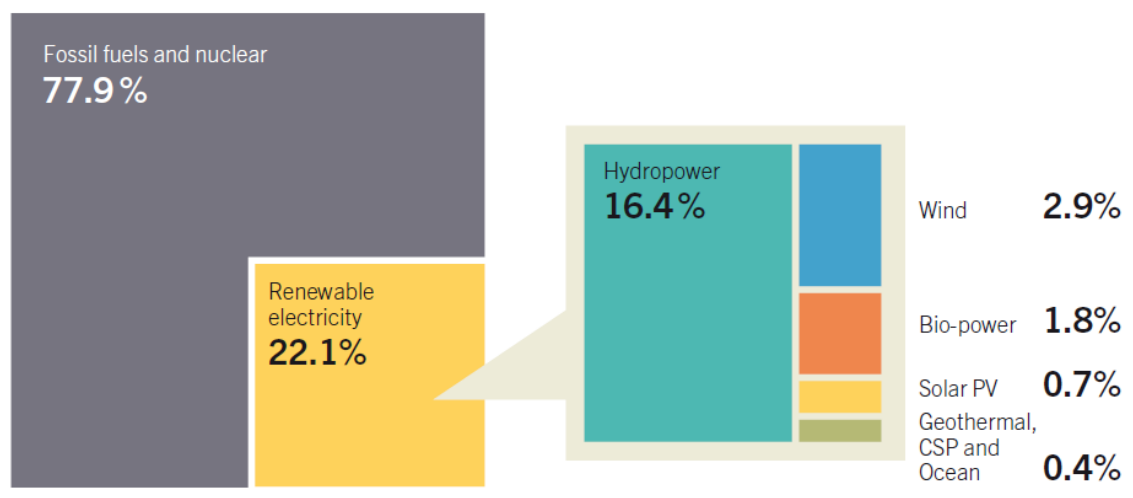

Figure 1-2: Renewable energy share of global electricity production in 2013 [2] 
The solar PV effect was first observed by the French scientist Alexandre Edmond Becquerel in 1839, where an electrode became conductive when illuminated by a light source [4]. In 1883, the American inventor Charles Fritts created the first working solar cell in the United States. This solar cell was made with a thin layer of gold (Ag) coated on a selenium (Se) layer. The conversion cell efficiency of this solar cell was about $1 \%$ [5]. The low cell efficiency and high material cost prevented the commercialization of Se solar cell. The history of the single-crystalline silicon (c-Si) solar cell started in 1918 when Jan Czochralski, a Polish scientist, discovered a method to grow single crystals of material, which greatly reduced the cost and the difficulty of fabrication [6]. From this time on, the c-Si solar cell became the primary material used for solar cells in research. On April 25th, 1952, one hundred years after Becquerel first observed the photovoltaic effect, the first practical c-Si solar cell in the world with approximately $6 \%$ total cell efficiency was fabricated in Bell Labs [7], making solar PV energy practical for power uses.

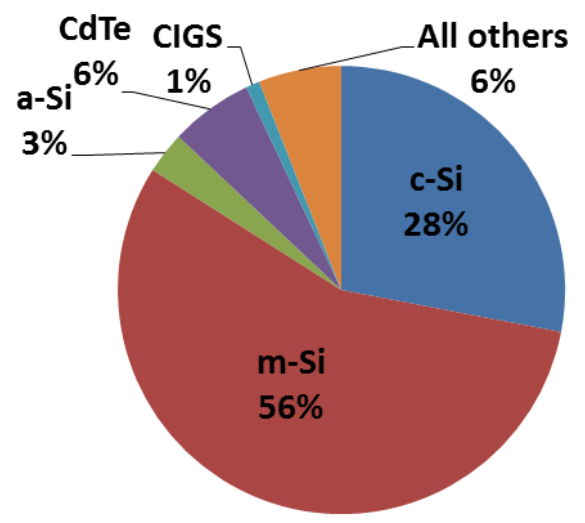

Figure 1-3: Solar PV module share by global development, END 2013[7]

Today, solar cells can be fabricated in a variety of forms of silicon, such as c-Si, polycrystalline silicon or multi crystalline silicon (m-Si), and amorphous silicon (a-Si). 
Fig. 1-3 shows the solar PV module technologies broken down by their share of development by the end of 2013. About $89 \%$ of solar cells used worldwide in 2013 for solar PV energy were derived from c-Si solar cells and m-Si solar cells. Specifically, mSi solar cells, which are the most commercially used solar PV sources in the global PV market, provided $56 \%$ of the global solar PV module market last year [8]. Currently, commercial silicon-based solar cells produce electricity at a rate that is competitive with the fossil fuel power plants in some regions of the world. However, the manufacturing and installation cost of solar cells needs to be lowered further to be commercially viable. The efficiency of the solar cells, either c-Si or m-Si vary as the fabrication process differs from each design. With elaborated high efficiency cell fabrication processes, the efficiency of c-Si solar cells can go up to $25 \%$. The average efficiency of c-Si solar cells was approximately $18 \%$ and the average efficiency of m-Si solar cells was approximately $10 \%$. The cells we make at Carleton usually have an efficiency of $\sim 4-5 \%$ due to the limitation of our fabrication capability. Reducing the manufacturing cost of solar cells is difficult since there are many different techniques and processes involved, in addition to various concerns: the costs of solar cell material, solar cell manufacturing facilities, and labor in these facilities.

According to Solarbuzz's "Solar PV Market Outlook" [8], solar PV energy shared only $0.7 \%$ of the global electricity production in 2013 , meaning the market share is 100 times less than that of fossil fuels. The commercial adoption of solar cells can only happen when the solar PV technology becomes cost completive enough to replace fossil fuels. The levelized cost of electricity from the solar PV technology was much higher compared 
to fossil fuel energy utilizing historical data [2]. In the current market, solar PV technology has yet to become cost competitive enough to replace fossil fuels.

In terms of reducing the manufacturing cost, increasing solar cell efficiency to reduce the electricity cost produced by solar cells is the most feasible approach, especially for silicon-based solar technologies. In the last few decades, many methods have been developed to enhance silicon-based cell efficiency, including the use of different materials to increase the solar absorption and wavelength range, the application of antireflection (AR) coating layers, and surface textured structures to reduce the front surface reflectivity of the cells. Unfortunately, cell efficiency enhancement is not enough to compensate for the price disadvantage. In order to further enhance solar cell efficiency, the feasibility of nanotechnology, including plasmonics nanoparticles and surface textured nanostructures for silicon-based solar cells, has been studied in this thesis.

By introducing nanotechnology into silicon-based solar cell application to enhance the electric field in the cells and to reduce the front surface reflectivity of the cells, the photocurrent generation rate and the light path length in the substrates can be enhanced to increase the overall cell efficiency. In addition, black silicon (b-Si) and horizontal-grown silicon nanowires (SiNWs) forming textured surface structures as two research avenues of nanotechnology have been discovered in recent years to effectively reduce or eliminate surface reflectivity. The b-Si structures modify the surface of silicon such that the surface has very low reflectivity, which makes it display a black color in the visible light spectrum. The horizontally-grown SiNWs structures, on the other hand, are small silicon wires of the nanometer scale that horizontally-grown on the silicon solar cell surface, compared to the vertically etched b-Si structures. Solar cells with the silicon nanowire 
structures on the top will also have low reflectivity due to the light diffused ability from the structures.

In the thesis, we will present a detailed study of the effect of the size and shape of the MNPs on the resulting efficiency of the c-Si solar cells, considering hemispherical and cubic shaped particles. In most reported experiments in the literature the preferred configuration of MNPs are hemispheres. However, we have proved in this thesis that the cubic configuration is a much more efficient shape. To the best of our knowledge, our group was the first group which utilizes the fundamentally different MNPs for solar cell efficiency enhancement. 


\subsection{Thesis Objectives}

This thesis presents the investigation of two main approaches that will enhance the total cell efficiency of single crystalline silicon based (c-Si) solar cells: (1) utilizing plasmonic nanotechnology by depositing metal nanoparticles onto the cell surface and (2) implementing surface textured nanostructures by employing the $b-S i$ and the horizontally-grown SiNWs onto the cell surface. The goal of the first approach is to study and optimize cell efficiency enhancement by introducing metal nanoparticles (MNPs) to c-Si solar cells. The design, fabrication, and characterization of the b-Si and the horizontally-grown SiNWs on c-Si solar cells are covered in the second section.

Chapter 2 provides the literature review and background of the thesis. The introduction and background on c-Si solar cell are presented at beginning of this chapter. Published information and optional functions of plasmonics nanotechnology for c-Si solar cell efficiency enhancement are then discussed. Next, we explain past research from literatures on the surface treatment for cell efficiency enhancement and experimentation on two types of surface textured nanostructures, including the b-Si structures and the SiNWs. At the end of each section, recent progress in plasmonics nanotechnology as well as the $\mathrm{b}-\mathrm{Si}$ and the silicon nanowire fabrication methods are highlighted.

Chapter 3 reviews the theoretical studying of MNPs and b-Si on solar cells based on the simulation results. The theoretical design of SiNWs structures is based on the reductive surface reflectivity mechanism. Detailed theoretical investigations through a numerical finite difference time domain (FDTD) simulation to determine plasmonicenhanced cell efficiency in a p-n junction c-Si solar cell are then covered. The optimization of metal nanoparticles designs to further enhance the cell efficiency, 
investigation of using silver (Ag) nanoparticles (NPs), and theoretical design for the b-Si structure fabrication process conclude the chapter.

Chapter 4 first outlines methods and procedures for fabrication of a p-n junction c-Si solar cell in the Carleton University MicroFabrication Facility (CUMFF). This solar cell is used as a reference solar cell and has the same structures as the solar cells with deposited MNPs. A brief explanation of MNPs on c-Si solar cells is also discussed after the c-Si section. Then, the detailed fabrication process for $\mathrm{b}-\mathrm{Si}$ structures on a c-Si wafer is explicated. Lastly, the epitaxial growth process of SiNWs structures on c-Si substrates using molecular beam epitaxy technology is analyzed in detail.

Chapter 5 first demonstrates the experimental results before and after MNPs were deposited on the top surfaces of the fabricated c-Si solar cells. The second part of the chapter explains the experimental results on two types of surface textured nanostructures, the b-Si structures, and the SiNWs structures with different fabrication conditions, such as reaction time and start conditions, among others. In order to characterize the efficiency enhancement, a scanning electron microscope (SEM) is mainly used to monitor changes in b-Si structures before and after the etching process. The photoluminescent (PL) results for the b-Si structure are used to analyze the electrical properties of the b-Si structure to verify the cell efficiency enhancement from the b-Si structures. At the end, the experimental results for c-Si solar cells with SiNWs structure on the front are explained. The surface reflectance and the current-voltage measurements results will be used to evaluate the enhancement of SiNWs structures on c-Si substrates.

To conclude the dissertation, Chapter 6 summarizes the present work of plasmonic nanotechnology, and the two types of surface textured nanostructures, b-Si and SiNWs, 
for the efficiency enhancement of c-Si solar cells. Future prospects of using Ag MNPs and b-Si and SiNWs structures as cell efficiency enhancement solutions are discussed at the end of the chapter. 


\section{Literature Review and Background}

According to Fig. 1-3 presented earlier, silicon-based solar cells (c-Si, m-Si, and a-Si), CdTe solar cells, and CIGS solar cells are the three major solar cell materials used in the solar cell market around the world. Together, they occupied $99 \%$ of the global solar PV market in 2013 [2]. Silicon-based solar cells have two main advantages compared to CdTe and CIGSs solar cells. Firstly, they are much cheaper because silicon ( $\mathrm{Si})$ is the eighth most common element in the universe and the second most abundant element in the earth. Secondly, they have fewer requirements and high tolerance in fabrication, which have led them to possess the highest market share in the worldwide solar cell market. Since silicon-based solar cells have advantages compared to solar cells made by other materials, enhancing cell efficiency of silicon-based solar cells has become an interesting topic worldwide. Many methods have been used on silicon-based solar cells to improve their cell efficiency since the first commercial silicon solar cell was developed by IBM.

In many cell efficiency enhancement solutions, nanotechnology applications on c-Si solar have caught lots of attention in the last few decades due to their advantages of enhancing incident light scattering and reducing reflectivity. Two directions of nanotechnology applications, plasmonic metal nanoparticles (MNPs) and surface textured nanostructures, on c-Si solar cells will be demonstrated in the thesis, respectively. The surface textured nanostructures application including two individual projects, which are black silicon (b-Si) nanostructures and horizontally-grown silicon nanowires (SiNWs). 


\subsection{Single-crystalline Silicon Solar Cell}

The bulk single-crystalline silicon (c-Si) solar cell is one of many silicon-based solar cells. The c-Si solar cell has an ordered crystal structure with each atom ideally placed in a pre-determined position. In this thesis, the c-Si solar cell is the substrate which will be used for the nanotechnology applications due to its many advantages. First of all, the c-Si solar cell exhibits better predictable and uniform behavior than other silicon-based solar cells during experimentation, which allows it to match most of the theoretical and technique developments required in the photovoltaic field. Secondly, it has the highest efficiency rate in all silicon-based solar cells given the standard condition that they are made out of the highest purity silicon. Thirdly, the c-Si solar cell has the longest lifetime than other silicon-based solar cells due to its high purity level.

Due to the advantages of c-Si solar cells, they have been used in the research facilities for high performance solar cell research for many years. However, the cost of c-Si solar cells is higher than other silicon-based solar cells even though it has the highest cell efficiency recorded. Cell efficiency enhancement has been a challenge for silicon-based solar cell designers for many years and is still of interest.

In terms of optical properties, the c-Si wafer has an average surface reflectivity approximately $40 \%$ from $200 \mathrm{~nm}$ to $1200 \mathrm{~nm}$ wavelengths [9]. The reflectivity of c-Si solar cells affects cell efficiency by reducing the number of photons absorbed by the substrate. Numerous techniques have been utilized to diminish the reflectivity, but all of them have different kinds of limitations on reducing reflectivity. Reducing the reflectivity of c-Si solar cells has become a predicable method to enhance the cell efficiency of c-Si solar cells. A distinct advantage for c-Si solar cells is that the thickness of c-Si solar cells 
allows for the building of diverse surface structures on the substrate. On the other hand, a thin-film silicon solar cell with the same reflectivity as the c-Si solar cell is not able to support any textured structures on the surface to reduce its reflectivity.

The junction type of silicon-based solar cell is important since it influences cell efficiency by changing the junction type of the solar cell. P-n and p-i-n junctions are conventional junction types used for silicon-based solar cells. The c-Si solar cells used as the Ag MNPs substrates are the p-n junction solar cells, which mean that a thin n-type layer is deposited on top of a thick p-type silicon wafer to form a p-n junction solar cell. For the $\mathrm{p}$-n junction solar cell, the electrons in the n-type region diffuses towards the $\mathrm{p}$ type region, and the holes in the p-type region diffuse towards the n-type region. As the diffusion process exposes holes in the n-type region and electrons in the p-type region, a special region is created around the junction interface, where there are a few free carriers compared to the n-type and the p-type regions. This region is called the depletion region, and an electric field forms in this region to keep the holes and electrons separated. For the p-i-n junction solar cell, the main difference compared to the $p-n$ junction solar cell is an intrinsic layer has to be inserted in between the p-type and n-type regions. This extra layer works as a larger depletion region to enhance photon absorption of solar cells.

The c-Si solar cell is a diffusion-based device due to its transport mechanism for photo-generate $\mathrm{d}$ carriers. The junction types used on the c-Si solar cell are flexible, which means that the c-Si solar cell could be a p-n or p-i-n junction solar cell. On the other hand, an amorphous silicon solar cell is a drift device, meaning that the a-Si solar cell must have an intrinsic layer in the solar cell for light absorption. The a-Si solar cell can only fix the p-i-n junction structure, which increases the fabrication cost by adding an 
extra layer. Hence, the c-Si solar cell is more predictable than the a-Si solar cell in the way of junction structure.

\subsection{Plasmonic Metal Nanoparticles}

Due to the development of modern technology, newer techniques have been invented within the last few decades and applied to different research areas to analyze their effects. Nanotechnology, devised in the early 1980s, is engineering and system analysis at the nanometer scale. This is a relatively recent scientific development, and it plays an important role in the progress of experimental advances in various fields, such as in the invention of the scanning tunneling microscope in 1981 and in the discovery of fluorescence in 1985 [10].

In the research field of optics, nanotechnology occupies a more important position. Nanotechnology is a bridge between the ordinary system, which relies on human observation and the nanoscale system, which involves particles of any shape with dimensions in the nanometer range. In the ordinary system, physical properties and optical properties of particles are not modified based on a change in size. In the nanoscale system, physical properties, such as the optical properties of material, change as the material sizes approach the nanoscale. Hence, scientists are able to control and enhance the optical properties of materials in a nanoscale system by nanotechnology. MNPs are one of many solar cell applications of nanotechnology; they play a significant role in enhancing solar cell optical properties.

One of the advantages of applying nanoparticles to solar cells mentioned herein is the ability to scatter incident light to different angles when light illuminates a metal surface. As incident light scatters through the nanoparticles at different angles, light path length 
through the solar cell substrate is increased. Another advantage of integrating nanoparticles into solar cells is the ability to enhance the localized electric field around the nanoparticles. If the nanoparticles deposit on the solar cell, the electric field inside the cell will be affected by the enhanced localized electric field from the nanoparticles, which results in an increase in photon absorption in the cell. The short circuit current increases as the number of photons translated to electrons in the cell increases.

In thin film silicon-based solar cells, due to the fact that the thickness of the solar cell is less than the height of the structures, using surface textured structures to reduce surface reflectivity are not applicable. In 2007, Professor Kylie Catchpole and her team at the Australian National University discovered that nanoparticles deposited on the top surface of a thin-film solar cell were able to enhance light path length without a surface textured structure [11]. Their simulation and experimental results support that the deposited silver nanoparticles on the front can enhance the efficiency of silicon-based solar cells. Moreover, they discovered that, within the visible wavelength range, as the size of metal nanoparticles decreased, the efficiency enhancement increased due to the increasing light path length through the cells.

Kylie and her group conducted many studies on integrating metal nanoparticles (MNPs) onto silicon-based solar cells. After they discovered that silver (Ag) MNPs can improve solar cell efficiency by increasing the light path length, they investigated the effect of MNP shape and type of materials on solar cell efficiency in 2008 [12]. For MNP shape, their results show that MNPs with a hemispherical shape have better efficiency enhancement compared to MNPs with other shapes, like triangles, cylinders and perfect spheres. Their results on types of NPs material show that Ag MNPs have more effective 
light path lengths than gold ( $\mathrm{Au}) \mathrm{MNPs}$ because Ag MNPs scatter more light into solar cells than Ag MNPs. Hence, Ag MNPs have better efficiency enhancement than Au MNPs. Based on their research, the light scattered by MNPs is also affected by the distance between each of them.

The light absorption coefficient of c-Si solar cell shows that the silicon-based solar cell must be greater than $1 \mathrm{~cm}$ thickness in order to absorb photons at $1000 \mathrm{~nm}$ wavelength. The thickness of solar cells is a major influence on solar cell efficiency since the thickness determines how many photons can be absorbed by the solar cell to produce electricity. Owing to existence of the surface plasmonic resonances on MNPs, MNPs can have more than one way to enhance the electrical properties of silicon-based solar cells. Depending on the position of the MNPs, there are at least three ways to increase light path length in solar cell structures while keeping their physical thickness constant [10]. The first approach is to deposit MNPs on the top surface of the solar cell so that they can be treated as a subwavelength scattering substrate to trap incident light into the solar cell. Second, when MNPs can be placed at the junction between the p-type and the n-type region, the second method is to make them subwavelength antennas to increase the effective absorption cross-section of the solar cell between the two regions. The third way to apply MNPs on solar cells is to place them near conductor inside the solar cell, in which they can be treated as textured structures on the bottom to scatter incident light at different angles. According to research from Atwater et al, depositing metallic nanoparticles on the top surface of the solar cell optimizes solar cell efficiency [10].

Due to the fabrication process of MNPs, it is very hard to change the shape of MNPs during an experiment. A hemisphere is the most commonly used MNP shape in research 
experiments since it is the easiest shape to fabricate on solar cells. However, a hemisphere is not the optimal MNP shape to generate surface plasmonic resonance in these cells. In 2012, A. Bottomly et al. published their research on fabricated cubic MNPs and discussed the optical properties of cubic MNPs on silicon substrates [13]. The research results demonstrated that cubic MNPs are able to generate stronger front electric fields compared to spherical MNPs [13]. Since increased electric field intensity around MNPs is one of many features of plasmonics nanotechnology on c-Si solar cells, depositing cubic MNPs will yield better cell efficiency enhancement than depositing spherical MNPs. The cubic silver MNPs synthesized by our chemical process are limited to have an edge length of $110 \mathrm{~nm}$. Any edge length above $110 \mathrm{~nm}$ for the MNPs will mostly lose their cubic shape.

The absorption of c-Si solar cells occurs between $200 \mathrm{~nm}$ and $1100 \mathrm{~nm}$ in wavelength. A c-Si solar cell has a higher absorption coefficient at short wavelengths compared to long wavelengths. Therefore, MNPs with a resonance wavelength at a short wavelength range better enhance the electrical properties of solar cells. The resonance wavelength of MNPs depends on the size, shape, particle material, and refractive index of the surrounding medium. The materials of MNPs applied on c-Si solar cells are required to have plasmon resonances at or close to infrared range because of the absorption coefficient of c-Si. Conventional materials for MNPs application are alumina (Al), silver (Ag), and gold ( $\mathrm{Au}$ ) because they have plasmon resonances at $155 \mathrm{~nm}, 350 \mathrm{~nm}$, and 480 $n m$ respectively when they are surrounded by air [14]. Al MNPs have plasmon resonances close to the ultraviolet range and are commonly applied on amorphous silicon and nanocrystalline silicon solar cells [15]. Since Ag MNPs have a better scattering 
cross-section at low wavelengths compared to that of the Au MNPs, applying Ag MNPs to c-Si solar cells better improves cell efficiency at short wavelengths [11]. The plasmon resonances of $\mathrm{Au}$ and $\mathrm{Ag}$ can be redshifted in a controlled way over the entire 500 to1500 $n m$ spectral range by changing the surrounding matrix [16] by designing the size, shape, and refractive index of the surrounding medium, and optimizing the resonance wavelength can maximum scattering ability from the MNPs. In Chapter 3, the effects of size, shape, and refractive index of the surrounding medium on c-Si solar cell efficiency are investigated.

\subsubsection{Surface Plasmons Resonance}

Surface plasmons resonance (SPR) is the collective oscillation of electrons in metal stimulated by illumined light. The SPR condition is established when the frequency of illumined light photons matches the resonance frequency of metal. Therefore, SPR is found in materials that have negative real and small, positive, imaginary dielectric constants, such as noble metals (for examples, silver (Ag) and gold (Au)) [17]. SPR polariton is comprised of electromagnetic excitations propagating at the interface between a dielectric and a conductor, in which the propagation is confined in the perpendicular direction. These electromagnetic surface waves appear through the coupling of the electromagnetic fields to oscillations of the conductor's electron plasma [18]. Fig. 2-1 shows the SPR polariton established by the incident light propagating at the interface between a dielectric and a metal. SPR is sensitive to incident light and the surrounding dielectric environment and can be used on different optical devices, like solar cells [17]. 


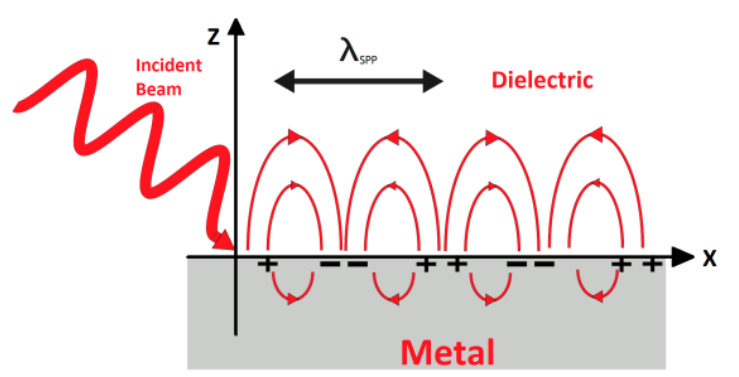

Figure 2-1: The SPR polariton propagating at the interface between a dielectric and a metal

Localized surface plasmons resonance (LSPR) is a variation of SPR. LSPR is surface plasmons resonance confined to a metal nanostructure much smaller than the wavelength of illuminated light [19]. The plasmons resonance localizes around the nanostructure with a special frequency called the LSPR frequency. LSPR is sensitive to the surrounding dielectric environment and metal nanoparticle geometry. Due to the successful development of nanoparticle fabrication techniques in recent years, nanoparticles enhancing solar cell electrical properties via LSPR has become a popular topic in solar cell applications. Fig. 2-2 shows LSPR polariton established by incident light propagating on the nanoparticle surface. When light is illuminated on metal nanoparticles, the conduction electrons are excited by incident photons. Then, movement of charges in the nanoparticle leads to a build-up of charge on the particle surface. The build-up charge on the surface acts as a restoring force, creating a resonance condition for electrons. Hence, the resonance conduction on metal nanoparticle is LSPR [19].

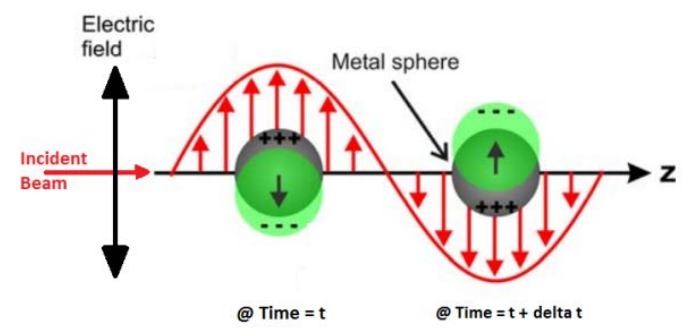

Figure 2-2: The LSPR polariton propagating at the nanoparticle surface 


\subsubsection{Light Scattering and Absorption}

At beginning of the twentieth century, Gustav Mie developed a description for light scattering and absorption properties of a spherical particle called the Mie theory [18]. The Mie theory or Mie scattering is a formulation for handling particle-light interaction problems for a spherical particle. In this section, particle-light interaction for a spherical particle surrounded by both nonabsorbing and absorbing mediums is expressed using the Mie theory [18].

\section{Arbitrarily shaped particle}
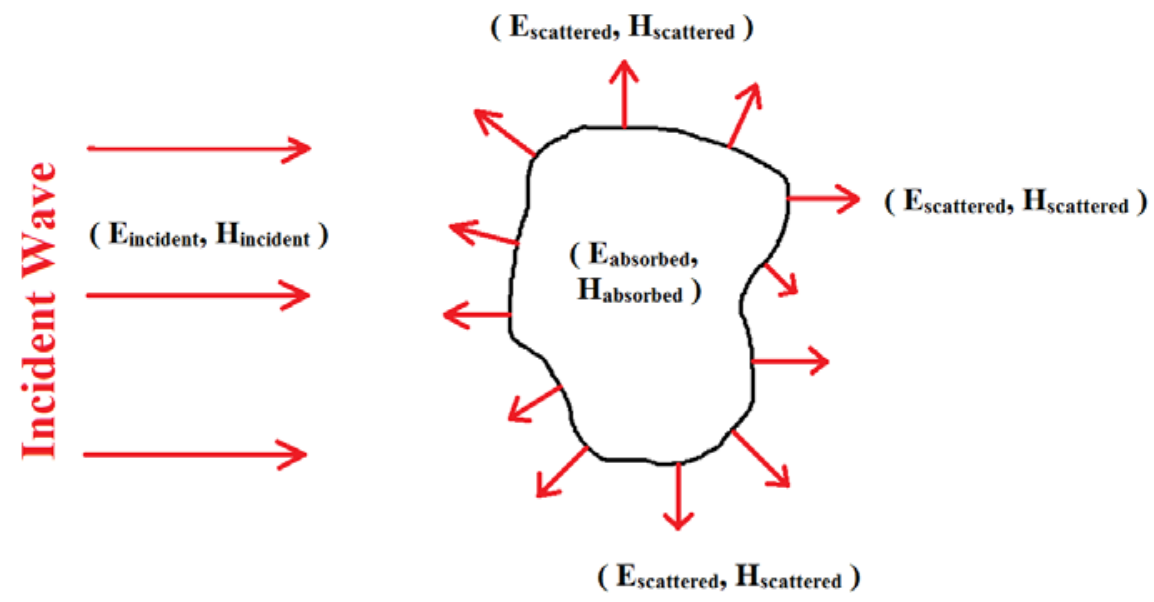

Figure 2-3: Geometry for incident wave scattering by particle of arbitrary shape

Fig. 2-3 above demonstrates schematically what appears when incident wave impinges on an arbitrarily shaped particle. The incident electromagnetic field ( $\left.\mathrm{E}_{\text {incident }}, \mathrm{H}_{\text {incident }}\right)$ is partially absorbed in substrate generated absorbed electromagnetic field and partially scattered from the substrates generated scattering electromagnetic field around the surface. When light is exposed on the spherical particle surface, the charges are set in oscillation, where the main effects appear in the emission of secondary radiation known as scattering. While scattering occurs on the particle, partial incident light may be 
recombined with the particle, provided that it absorbs. The combination of scattering and absorbing explains the temporal implication of reduction of the incident light after being exposed to a spherical particle. Extinction is defined as the net affection of radiation extinguished from incident light penetrating the particle [20]. By using the Mie theories and relevant equations devised from the Mie theories, the absorption, scattering, and extinction efficiencies for a spherical particle surrounded by a dielectrical medium can be expressed as equations with the rate of energy incident on the particle. Appendix A includes analytical studies of the Mie's solutions and their extension equations.

When the wavelength of incident light is close to the resonance wavelength of the MNPs, photons from the incident light are scattered or absorbed by the MNPs. Based on Mie's solutions and their extension equations, scattering and absorption efficiencies for MNPs depend on the size of MNPs. When the size of MNPs is much smaller than the wavelength of light injected to the MNPs, MNPs absorb more photons and then scatter, which means that the absorption effect is dominated in the extinction process. When the size of MNPs is close to the wavelength of incident light, MNPs have a stronger scattering ability to scatter more photons to surrounding mediums compared with absorption ability. However, the size of MNPs has to be beyond certain limits to maintain their working functions for LSPR. Increasing the size of MNPs also increases their retardation effects, which decrease the scattering ability of MNPs. 


\subsubsection{Black Silicon Nanostructures}

One of the most important aspects to improving overall cell efficiency is reducing the front surface reflectance of crystalline. Compared with a $100 \%$ reflected mirror, polished silicon wafers have surface reflectivity from $70 \%$ to $30 \%$ depending on the wavelength of incident light [9]. In short wavelength ranges, silicon wafers appear to have a higher average reflectivity and a higher average absorption coefficient than for longer wavelength ranges. In this case, reduced reflectivity in wavelength regions with high absorption will significantly enhance on cell efficiency.

Reduced front surface reflectivity is a commonly used process in modern solar cell fabrication. AR coating and surface textured structures are the most often employed technologies to reduce surface reflectivity. AR coating technology is deposited on a lower reflectivity layer on the front surface of the solar cell to reduce reflectivity at specific wavelengths [21]. However, the layer can only reduce reflectivity at specific wavelengths, which is not sufficient when solar cells are excited under the whole visible light spectra. A flat surface has higher reflectivity than a textured surface since more light can be instantly reflected from the surface. The surface textured structure technology uses a chemical solution to etch the front surface of the solar cell to reduce reflectivity. Due to accurate chemical etching technology, the surface textured structures are capable of fabricating texture structures at the micron scale [22]. Black silicon (b-Si) structures are one type of surface textured structure. Compared with normal surface texture structures, the black silicon structures are smaller and have a larger distribution angle to incident light. Therefore, fabrication of black silicon structures has to be at the nanometer scale to reach the above features. Furthermore, the heating effect caused by the b-Si structures on 
the front surface of the solar cell should be minimal because the unwanted photons have wavelengths longer than the bandgap wavelength of Si. Consequently, the long wavelength photos will not "see" the small b-Si structures and will directly pass through the solar cell.

\subsubsection{Reduced Surface Reflectivity}

Black silicon (b-Si) structures feature needle-like surface structures, where needle-like shaped structures are made of c-Si material. The b-Si solar cell is a c-Si solar cell that has the b-Si structure on the front surface. The reflectivity of untreated c-Si solar cell reduces the amount of photons injected into the solar cell, which decreases the cell efficiency at the same time. The average reflectivity of an untreated c-Si solar cell is around $40 \%$. By using the b-Si structures on the front surface, the front surface reflectivity of the c-Si solar cell can be controlled below $20 \%$, which means that an additional $20 \%$ of photons from incident light are injected into the solar cell substrate compared to the conventional c-Si solar cells [23].

The b-Si structures on the c-Si solar cell front surface can reduce the reflectivity according to diffraction effects and the "moth-eye effect" occurring under incident light. The reflectivity of c-Si solar cells is due to the abrupt transition of light from the surrounding medium to the solar cell. Increased refractivity difference between the solar cell and the surrounding media will increase reflectivity on the solar cell-surrounding media interface. The interface reflectivity can be reduced when the disparity between two mediums is decreased. The b-Si structures on the solar cell are constructed at the micron scale so that the geometry of b-Si structures can reduce the reflectivity at different wavelengths by making a continuous transition between two materials [24]. When 
incident light is in a shorter wavelength region, the diffraction effects occur at the b-Si structures since the dimensions of the needle-like structures are comparable to the wavelength of incident light. As the incident light has been diffracted through the b-Si structures, the incident light can be diffracted to different angles, which would increase the path length of light in the substrate. When the incident light is at a longer wavelength region, the moth-eye effect occurs at the b-Si structures since the dimensions of the needle-like structures are close to the wavelength of incident light. The surface on the bSi structures behaves like an effective medium, and the effective index of refraction changes continuously from that of the surrounding medium to that of the needle-like structures. Hence, the reflectivity on the b-Si structures is lower than the reflectivity on conventional c-Si solar cells [25].

However, the lower reflectivity compared to other types of silicon doesn't make the overall efficiency of b-Si solar cells higher than the others. It is because the overall efficiency depends not only on reflectivity, but also surface recombination and other associated effects. Unfortunately the etcher in the fabrication facility broke down beyond repair since April 2014 (hence the delay in my thesis defense), we were unable to create full silicon cells integrated with b-Si structures. In order to demonstrate the potential of the b-Si structures, I performed a series of characterization methods, such as reflectivity measurements and photoluminescent measurements to estimate the surface recombination effect. The conclusion from the characterization of the b-Si structures was that b-Si structures are promising. 


\subsubsection{Fabrication Procedure}

Black silicon technology generates pyramid-like or needle-like structures on the surface of the substrate and can be an alternative or complementary to AR coating to further reduce optical losses. Texturing the front surfaces of solar cells improves cell efficiency by increasing the short circuit current $\left(\mathrm{I}_{\mathrm{SC}}\right)$. This increase is due to higher absorption of photons closer to the p-n junction and entrapment of photons in the cell. Light trapping becomes more important as the solar cell gets thinner.

The etching techniques used in surface texturing can be classified into two groups: wet etching and dry etching. The former is based on gas mixtures, whereas the latter relies on chemical solutions. In comparison to wet chemical etching, the dry etching creates directional etching without using the crystal orientation of silicon substrate. Although the dry etching methods provide better process control, they require more expensive equipment and may cause re-deposition of compounds on the wafer surface. The wet etching method is based on preferential etching along certain grain orientations, and it can be performed by alkaline or acidic solutions.

In order to achieve light trapping and surface reflectance comparable to that of mono$\mathrm{Si}$, several methods have been developed for multicrystalline silicon. Zhao et al. reported a honeycomb like structure that was obtained by alkaline wet chemical etching through a photo resist mask [9]. Some researchers were able to achieve a surface structure with a porous silicon layer by using maskless acidic texturing [26]. Since the early 1990s, various dry surface texturing methods, such as laser-structuring, mechanical diamond saw cutting, and plasma-based etching, were developed. Inomata et al. developed the first solar cell with a surface texturing from a chlorine plasma without using any etch mask 
[27]. Although chlorine offers a slow etching rate and a better profile control, chlorine based plasma etching requires higher safety standards and expensive equipment due to its toxic and explosive nature, which is why chlorine is not commonly used in plasma etching. Today, fluorine based plasma is the preferred method because it has chemical properties very similar to that of chlorine and it is easy to handle and harmless for operators. In reactive ion etching (RIE), a grass-like structure known as b-Si structures occurs at the bottom of deep trenches. Jansen et al. in 1994 first observed b-Si structures as an undesirable side effect of RIE [28]. Later, they developed a recipe for b-Si. Following this method, it is possible to develop a plasma etch process that produces homogeneous black silicon surfaces structures on c-Si wafers. The following section gives a short review on the main etching techniques.

\section{Wet Etching Method}

Wet etching techniques used in surface texturing can be classified into two main subcategories: metal assisted chemical etching (MACE) and electrochemical etching. MACE is implemented by depositing pre-made metal nanoparticles on the substrate, which are removed later by etching with a chemical solution of an oxidant and a complexing agent for silicon. The surface passivation is achieved by depositing $\mathrm{SiO}_{2}$ by a wet chemical deposition process. In comparison to dry etching methods, MACE does not require high energy use or complicated fabrication processes [23]. Electrochemical etching involves dipping a silicon substrate into a solvent and applying a low voltage current to the solution (electrolyte). This creates an oxide layer over the silicon, which is then extracted by another electrochemical process. The etching profile depends on the anode current density, electrolyte solvent, and anodization time. 
$\mathrm{KOH}$ and $\mathrm{NaOH}$ solutions are the most commonly used alkaline solutions for this purpose. The surface structure with alkaline wet chemical etching looks like inverted pyramids when photolithographic etch masks are used or random pyramids when a maskless process is employed. The main limitation of alkaline wet chemical etching is the fact that it only works well on c-Si wafers with $<100>$ crystal orientation. Wet chemical texturing with alkaline solutions is difficult to apply on c-Si wafers due to the significant amount of silicon loss. Isotropic etching in acid solutions is a commonly used technique for c-Si substrates.

\section{Dry Etching Method}

In general, there are two types of dry etching methods: non-plasma-based dry etching and plasma-based dry etching. Non-plasma-based dry etching relies on chemical reactions of reactive gas mixtures introduced into the etching chamber. Plasma-based dry etching uses radio frequency $(\mathrm{RF})$ power to generate plasma and induce chemical reactions. This method results in isotropic etching of silicon with high selectivity to masking layers. There are four basic types of plasma-based dry etching: physical (sputter) etching, chemical etching, RIE, and deep reactive ion etching (DRIE).

Physical plasma etching is based on bombardment of the wafer surface by accelerated ions, atoms, and molecules, which dislodge substrate atoms. In chemical plasma etching, the reactive species in plasma diffuse into the surface of the substrate, and then they are absorbed by the substrate. The etching is accomplished when the products of the chemical reaction between the absorbed reactive species and the substrate are released back into the plasma chamber. 


\section{Reactive Ion Etching Method}

The RIE method is a widely used etching technique, in which chemical plasma etching is accompanied by ion bombardment of the substrate. Ruby et al. have demonstrated the use of sulfur hexafluoride and oxygen $\left(\mathrm{SF}_{6} / \mathrm{O}_{2}\right)$ RIE for simultaneous texturing and emitter etching [29]. Most DRIE techniques employ an electron cyclotron resonance (ECR) source accompanying an RIE system. The major advantage of DRIE over RIE is its capability to control the energy and density of ions independently from each other. Hence, DRIE can achieve higher ion density with lower energy at lower pressures with a net effect of lower substrate damage and better selectivity. Another variant of DRIE is based on the use of inductively coupled plasma (ICP). An ICP system employs two independent RF sources, one to control the density and another to control the energy of the ions. Systems that rely on ECR and ICP create very similar etching profiles and process results.

RIE method uses a mixture of at least two gases, one of the gases in the mixture reacts with the silicon and creates an etched surface, while a second gas is used to passivate the etched surface. Typical gas mixtures used in RIE are $\mathrm{SF}_{6} / \mathrm{O}_{2}, \mathrm{SF}_{6} / \mathrm{Cl}_{2} / \mathrm{O}_{2}$, and $\mathrm{SF}_{6} / \mathrm{O}_{2} / \mathrm{CH}_{4}$.

The RIE process is based on both chemical and physical etching. Fig. 2-4 depicts the conventional RIE process via a $\mathrm{SF}_{6} / \mathrm{O}_{2}$ gas mixture. $\mathrm{SF}_{6}$ is used to create fluorine radicals $\left(\mathrm{F}^{*}\right)$ that react with the silicon. Under normal experimental conditions, the product of this reaction, $\mathrm{SiF}_{4}$, exists as a gas and is removed from the surface quickly. Meanwhile, oxygen radicals $\left(\mathrm{O}^{*}\right)$ react with the etched surface to passivate the sidewalls with $\mathrm{Si}_{\mathrm{x}} \mathrm{O}_{\mathrm{y}} \mathrm{F}_{\mathrm{z}}$ to stop further etching of the silicon. At the beginning of the RIE process, 
the etch rate is usually very slow because the silicon surface is covered with native oxide. Thus, initially, this layer is perforated by the bombardment of positively charged ions in the plasma. Once the silicon is exposed to the radicals in the plasma, the chemical etching of the wafer starts. The competing process of etching and $\mathrm{Si}_{\mathrm{x}} \mathrm{O}_{\mathrm{y}} \mathrm{F}_{\mathrm{z}}$ formation maintains the shape of the surface structure.

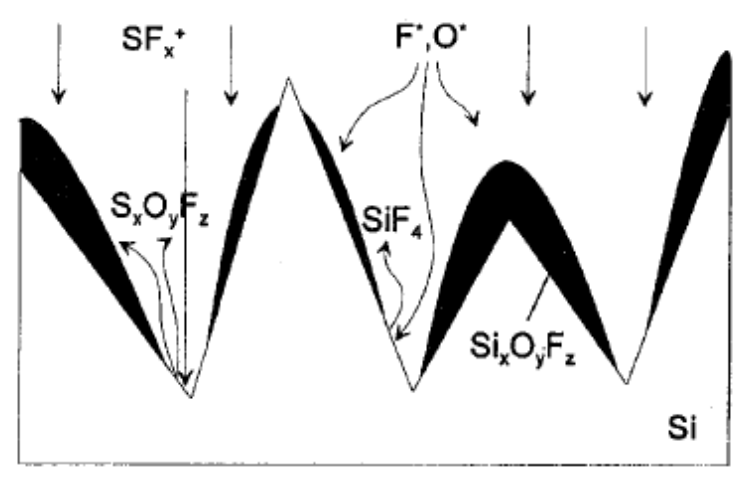

Figure 2-4: RIE process by $\mathrm{SF}_{6} / \mathrm{O}_{2}$ gas mixture [20]

The directed ion bombardment removes both the silicon and the $\mathrm{Si}_{\mathrm{x}} \mathrm{O}_{\mathrm{y}} \mathrm{F}_{\mathrm{z}}$ film. The optical and morphological properties of the etched silicon surfaces depend on the concentration of $\mathrm{F}^{*}$ and $\mathrm{O}^{*}$ radicals in the plasma, the strength of the adsorbed $\mathrm{Si}_{\mathrm{x}} \mathrm{O}_{\mathrm{y}} \mathrm{F}_{\mathrm{z}}$ layer on the sidewalls, and the working pressure of the plasma. $\mathrm{As}_{\mathrm{SF}}$ flow increases, the concentration of fluorine radicals in the plasma gets higher, forming volatile SiF4 and causing higher etch rates. Increasing $\mathrm{O}_{2}$ flow rate decreases the chemical etch rate due to increased passivation of the side walls and the bottom of trenches. At high $\mathrm{O}_{2}$ levels, the etching becomes more physical. The working pressure chosen for the plasma affects the surface structure of the silicon wafer by changing the density of the needle-like structures called grass. Other important parameters for RIE are the etching duration, the geometry of the plasma chamber, and the RF power used to generate the ions. 
Although the ion bombardment creates areas for chemical reactions, it results in lower selectivity. The main disadvantage of RIE is that as the etching rate increases with the concentration and pressure of the reactive species, leading to more collisions and anisotropic profiles. RIE has variations, such as inductively coupled plasma reactive ion etching (ICR - RIE) and damage-free reactive ion etching [23].

The RIE process generates defects on the surface of silicon wafers, which lead to high surface recombination velocity. The charge carriers have a higher chance of combining at these defect sites. RIE solar cells have lower blue response and short circuit current if the surface is not treated with damage removal etching.

\section{Electron Cyclotron Resonance Ion Etching}

Electron cyclotron resonance (ECR) ion etching method is also called the microwave plasmas ECR ion etching technique due to resonant microwave plasma discharged during the etching process. In Fig. 2-5, the left image displays the microwave ECR plasma etching machine in a nano-fabrication lab, and right image shows the construction of the ECR etching machine. A microwave radiation generated with a magnetron is introduced into the discharge area enclosed in a quartz tube in the machine. Then, an etching gas is introduced into the chamber below the discharge tube. In order to generate reactive ions from the etching gas, a mirror type magnetic field produced by three solenoids and a permanent magnet will enhance the microwave power deposition into the plasma. The reactive ions produced in the plasma will etch the substrate on the stage set below the discharge tube [30]. 


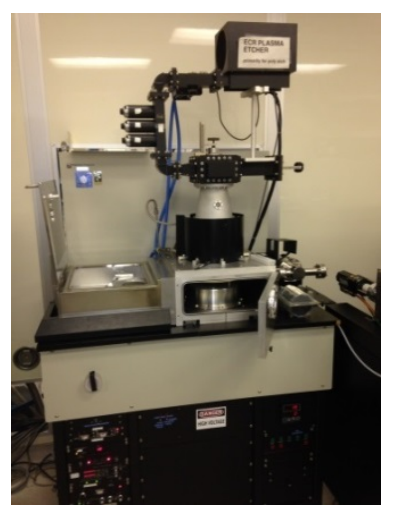

(a)

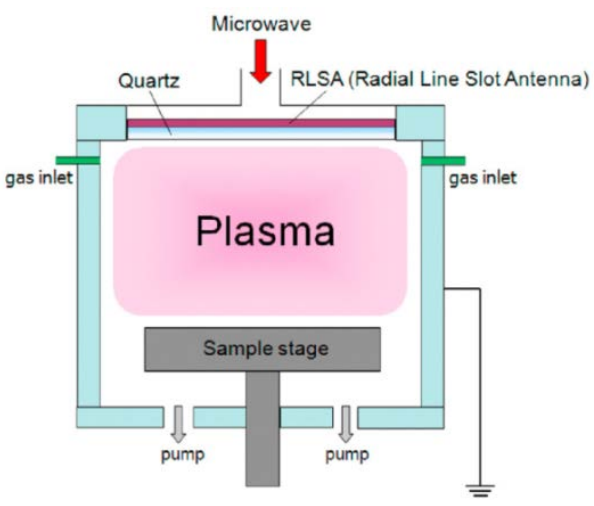

(b)

Figure 2-5: (a) Photo image of the ECR etching system in CUMFF (b) The construction of the ECR etching system

\section{Damage Removed Etching Method}

A disadvantage of a $b$-Si solar cell is high surface recombination caused by a larger surface area from b-Si structures. ECR etching creates the needle-like shaped structures and a large number of dangling bonds. These dangling bonds could work as defect structures on the needle-like structures to increase surface recombination rate with a large area. High recombination rates decrease solar cell efficiency by reducing the amount of electron-hole pairs in the substrate. These defect structures or roughness structures have to be removed from the black silicon solar cell without damaging the needle-like structures [31].

Kumaravelu et al. reported that RIE would create defects in nanostructures, resulting in a much larger surface area, which might decrease the minority carrier lifetime significantly [32]. Therefore, it is necessary to use a defect removal etching (DRE) process to improve the performance of black silicon solar cells. Usually, DRE is carried out by exposing the wafer to an acid mixture, such as $\mathrm{HNO}_{3} / \mathrm{HF} / \mathrm{HNO}_{3}$ forming an oxide film on the surface, which is etched by HF. The removal of oxide film by HF reduces both the density and the heights of the nanostructures on the surface. As the DRE time is 
increased, the number of needle-like structures decreases and the surface becomes smoother. Moreover, the reflectance of the RIE-textured surface approaches the reflectance of the acid textured surface. Thus, it is critical to determine the optimal etching duration to avoid a significant loss of nanostructures. Lee et al. fabricated black silicon solar cells with the RIE process followed by a DRE step [33]. The efficiency of these solar cells was as high as $16.32 \%$, which was higher than the efficiency of acidtextured solar cells. Yoo et al. proved that a DRE process could greatly improve the electrical performance of black silicon solar cells [34]. Xiao et al. tested six different DRE conditions and found that DRE process with $\mathrm{NaNO}_{2} / \mathrm{HF} / \mathrm{H}_{2} \mathrm{O}(6.4 \mathrm{~g}: 10 \mathrm{ml}: 240$ $m l$ ) produced the best performance for black silicon cells [35]. Applying the DRE process to black silicon solar cells produced by ECR etching could further enhance cell efficiency by reducing surface recombination. 


\subsubsection{Silicon Nanowires}

Horizontally-grown silicon nanowires (SiNWs) are popular building blocks for developing a new generation of electronics and photovoltaic devices, like photodetectors and solar cells [36]. In order to apply SiNWs in those devices, the precise position or orientation control of the SiNWs in a convenient planar architecture is a prerequisite. However, growing self-assembled SiNWs into precise locations on Si substrate with different orientation represents a critical capability to scale up SiNWs-based functionalities. There are two commonly used SiNWs, which are known to apply to silicon-based solar cells, vertically grown SiNWs and horizontally grown SiNWs. In the past few decades, research interests have been devoted to transferring vertically grown SiNWs via a vapor-liquid-solid (VLS) process onto planar substrates with the aid of over growth manipulation or by forcing them into growing horizontally by using channels defined on the substrate surface [37]. This is because horizontal or planar electric connection or circuitry still remains the most convenient architecture for prototyping various SiNW-based functionalities, like SiNWs application on silicon-based solar cells. Thus, a controlled in-plane growth of SiNWs is considered as an important opportunity to integrate large scale self-assembled SiNW functionalities across different devices.

The VLS growth process was first proposed by Wagner et al. in 1964 [38] and, to date, still remains the most utilized and debated paradigm for modelling the growth of vertical or horizontal SiNWs. The term vapor-liquid-solid refers to the pathway of silicon during the growth process. According to this process, silicon in the vapor phase impinges on a substrate covered with liquid metallic nanoparticles. Then, silicon atoms dissolve into the liquid droplets and, when their concentration in the droplet exceeds the saturation point, 
excess silicon crystallizes as solid nanowires at the droplet/substrate interface. In the VLS process, the cross-section and crystallographic direction of the nanowire's growth are controlled by the surface energies at the droplet/silicon interfaces and silicon/gas phase. The underlying assumption is that SiNWs growth takes place under local thermodynamic equilibrium conditions and in a very small volume close to droplet/wire interface [39]. Gold $(\mathrm{Au})$ is the typical metal of choice for the preparation of the droplets because it is chemically inert and has an eutectic point at a low temperature and high semiconductor solubility [40].

Based on the research from Weyher et al. [41], minimization of the Au droplet/silicon interface energy dictated that sufficiently large SiNWs preferentially grow along the $<111>$ silicon crystallographic direction with a hexagonal cross-section formed by $\{110\}$ and $\{112\}$ factors. Conversely, the lateral surface energy contribution dominates over the Au droplet/silicon interface energy for SiNWs and wires of a relatively small diameter. Even at the slowest growth rates, surface energy minimization controls the preferential direction of growth, which results in SiNWs that are oriented along the $\langle 110\rangle$ crystallographic direction, with hexagonal cross-sections formed by four $\{111\}$ and two $\{100\}$ facets [42]. According to research from Wu et al. [43], their high resolution transmission electron microscope experiments indicate that the gold/silicon interface remains oriented along the $<111>$ direction even during $<110>$ growth, with the formation of facets at the Au droplet/silicon interface.

The majority of the literatures on VLS growth of oriented SiNWs refer to nanowires that grow vertically. This requires further assembling procedures to make in-planar integrated-circuit devices on the substrates, like silicon-based solar cells. Based on our 
research, the few existing literatures of controlled horizontal growth of SiNWs describe their fabrication process in lithographically patterned trenches on $<110>$ orientation silicon substrates, whereas spontaneous horizontal growth is highly desirable for electronic application [39] [40]. Therefore, we demonstrate the theoretical design of arrays of horizontally growth SiNWs structure supplication on the c-Si solar cell in the thesis. The fabrication process and the experimental measurement results will also be discussed in this thesis. 


\section{Theoretical Study}

\subsection{Simulation Methodology}

In this chapter, a simulator package from RSoft is used to help simulate the theoretical design. The numerical analysis technique employed in the simulation package is the finite-difference time-domain (FDTD) simulator. Comparing to other numerical methods, the FDTD method can cover a wide wavelength range with a single simulation. The incident light absorbed and scattered by the silver (Ag) MNPs and substrates is easily determined utilizing this method since the FDTD method can cover the entire absorption wavelength for $\mathrm{c}-\mathrm{Si}$ solar cells. The FDTD method is able to simulate the propagation of light through any medium in two or three dimensions. In the plasmons simulation section, the FDTD simulator simulates the propagation of light through the Ag MNPs and the c-Si solar cell in a two-dimensional system to help increase simulation accuracy. For the b-Si simulation part, the FDTD method uses the two-dimensional system to simulate the optical properties of the b-Si structure. The mesh grid employing the b-Si simulation is smaller than in plasmons simulation section since the structure scale of the needle-like structures is much smaller than the size of the Ag MNPs.

The simulation results from the FDTD simulator are under ideal conditions, which mean that the front surface conductor shadowing influence and any effects from the surrounding medium are not involved in any simulated results through this simulator. Therefore, the simulated results that come from the FDTD simulator are ideal and are measured from the MNPs, the b-Si structures, and the c-Si solar cell only. In the FDTD simulator, a real-world three-dimensional structure will rephrase to a two-dimensional structure by changing its boundary conditions. The boundary conditions of the simulated 
solar cell module are set to be perfect matching layers (PML) for boundaries related to solar cell width and periodic layers for boundaries related to solar cell thickness.

\subsection{Single-crystalline Silicon Solar Cell}

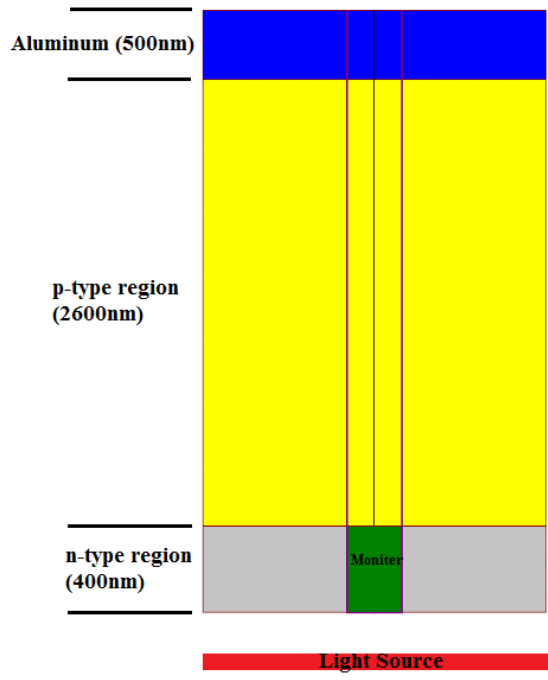

(a)

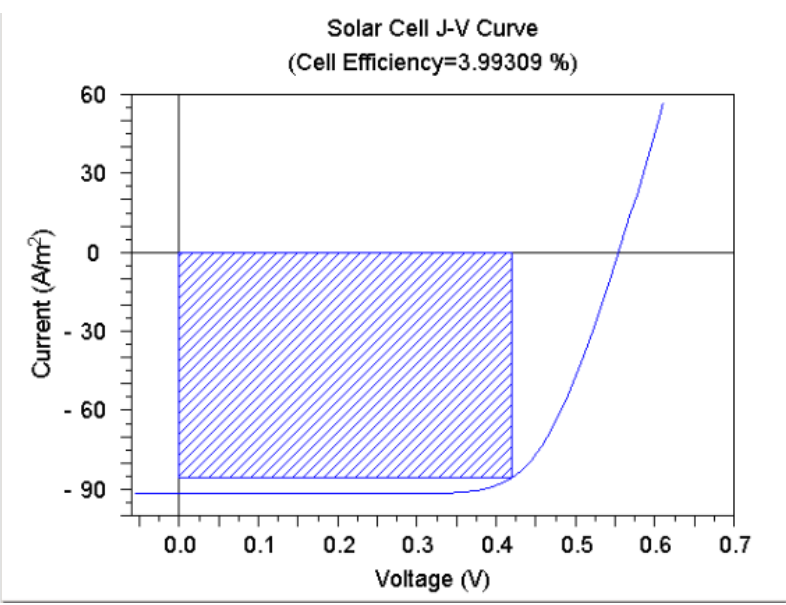

(b)

Figure 3-1: (a) A two-dimensional schematic view of a simulated p-n junction c-Si solar cell, (b) Simulation result for the c-Si solar cell build in the simulator

First, a c-Si solar cell is set up in the FDTD simulator as the reference solar cell for comparison. This c-Si solar cell module in the simulator can be used as a solar cell substrate to deposit MNPs in the plasmons simulation section. In order to ensure the accuracy of the solar cell module in the simulator, the parameters involved in the solar cell model are from the fabricated c-Si solar cell at CUMFF.

Fig. 3-1 (a) shows a two-dimensional schematic view of a p-n junction c-Si solar cell simulated in the simulator. The blue color region is the $\mathrm{n}$-doped region with a thickness of $400 \mathrm{~nm}$. The yellow color region is the p-doped region with a thickness of $2600 \mathrm{~nm}$. The light silver color region was the back conductor, which is alumina mater with a thickness of $500 \mathrm{~nm}$. The top conductor was not included in the simulator because it was 
only necessary for the device testing after fabrication. Incident light was illuminated in the n-doped region on the front surface of the solar cell at the standard test conditions. The dark green area in the schematic view was the monitor window used in the RSoft to measure the electrical properties of the solar cell. Due to the limitation of solar cell size in the RSoft simulator, the boundary condition of the solar cell on the horizontal axis was periodic and on the vertical axis was a perfect matching layer (PML). This solar cell structure was used in subsequent simulations with different nanoparticles and sublayers.

Fig. 3-1 (b) shows simulation results from the solar cell utility for the p-n junction solar cell explained in Fig. 3-1 (a). The cell efficiency of the single crystalline silicon solar cell was $3.99 \%$ with a fill factor of $70.6 \%$ and a collection efficiency of $72 \%$. The fill factor and collection efficiency came from a real c-Si solar cell fabricated in the micro-fabrication facility at Carleton University. The c-Si solar cell fabricated in the facility had the same doping level as the solar cell used in the simulation. By comparing the solar cell used in the simulation with the solar cell fabricated in the lab, we were able to set up the simulation parameters. Due to the limitation of the RSoft simulator, there are discrepancies in the choices of simulation parameters. For example, the thickness of the p-typed region in the fabricated solar cell was $500 \mu \mathrm{m}$, but the simulation uses $2600 \mathrm{~nm}$. Since we were looking at absolute efficiency enhancement from the metallic nanoparticles in simulations to come, the effect from the thickness difference between the real-world solar cell and the simulation solar cell was able to be ignored from the simulation results. 


\subsection{Plasmonic Nanotechnology}

The nanotechnology used for solar cell application can be extended to different applications, such as nanostructures, nanoparticles, nanoimprinted, in addition to nanophotonic light trapping via MNPs on the surface of c-Si solar cell, as verified in this thesis. The effect of Ag MNPs shape on plasmonic enhancement and efficiency enhancement will be first demonstrated. Next, the optimal size of Ag MNPs on c-Si solar cell will be investigated. Based on the literature review, MNP surrounding media will also affect plasmonic enhancement by changing refractive index. The third section of the plasmonic nanotechnology chapter will focus on the effect of Ag MNP surrounding media on plasmonic enhancement and efficiency enhancement. The Ag MNP surface coverage is the density of Ag MNPs distributed on the solar cell, and higher surface coverage means that more Ag MNPs have been deposited on the surface. The effect of Ag MNP surface coverage on efficiency enhancement will be investigated after. At the end, an optimization section will combine all simulation results together to find out the optimal shape, surrounding media and surface coverage of Ag MNPs for efficiency enhancement on c-Si solar cells.

The first step is in the plasmons technique in need of investigation is the material used to produce the MNPs. In order to generate LSPR on the MNPs, the MNPs have to be fabricated from metals with resonance wavelength in the c-Si solar cell absorption spectrum. There are three metals that are conventionally used in the photonics investigation that meet this requirement: silver $(\mathrm{Ag})$, gold $(\mathrm{Au})$, and aluminum $(\mathrm{Al})$. By comparing the dielectrical function and resonance wavelength of these three metals, the absorption cross-section of Au MNPs has the highest absorption cross-section among the 
three of them. With the same incident power, the MNPs with a higher absorption crosssection have a less scattered cross-section, which reduces the enhancement of cell efficiency from the MNPs. It is well-known that the c-Si solar cell has a higher absorption coefficient short wavelength range than a long wavelength range. The resonance mode at the resonance wavelength is red-shift by using the Al MNPs to replace the Ag MNPs. This red-shift of the resonance mode is due to retardation effects caused by the resonant condition from Al MNPs. However, the red-shift of the resonance mode is not good when the MNPs are applied to the c-Si solar cell due to the wavelength spectrum of the c-Si solar cell absorption coefficient. The Ag MNPs used on the c-Si solar cell show the best scattering ability and enhancement than MNPs made by other metals. Another advantage of using Ag as the metal of the MNPs is that cubic Ag MNPs show better fabrication tolerance than cubic Au MNPs in the lab. Furthermore, using Au MNPs on the c-Si solar cells will remove the minority carriers to promote the electronhole recombination. 


\subsubsection{Effect of Ag MNPs Shape on Plasmonic and Efficiency Enhancement}

Due to the local surface plasmon resonance (LSPR) effect by incident light, the MNPs were able to enhance the forward electric field and the scattering light into the solar cell. LSPR consisted of the collective oscillations of the conduction electrons localized around the nanostructure with a specific frequency. The resonance frequency of Ag MNPs was specific, which allowed the resonance to perform on the Ag MNPs surface depending on the size, shape, particle material, and refractive index of the surrounding medium. In this section, the effects of Ag MNPs geometry were investigated. The hemisphere was conventional in terms of nanoparticle geometry used in solar cell research, which was easy to synthesize in the lab with low-level device requirements. By changing the shape of Ag MNPs, we found that solar cells with cubic-shaped MNPs had higher backward electric field intensity at the interface than solar cells with hemispherical shaped Ag MNPs.

\section{Effect on Plasmonic Enhancement}

Fig. 3-2 below shows the simulation results for cubic Ag MNPs and hemispherical Ag MNPs; Fig. 3-2 (a) presents the electric field distribution for cubic Ag MNPs deposited on the front surface of c-Si solar cells, and Fig. 3-2 (b) shows the electric field distribution for spherical Ag MNPs deposited on the top of the c-Si solar cell. 


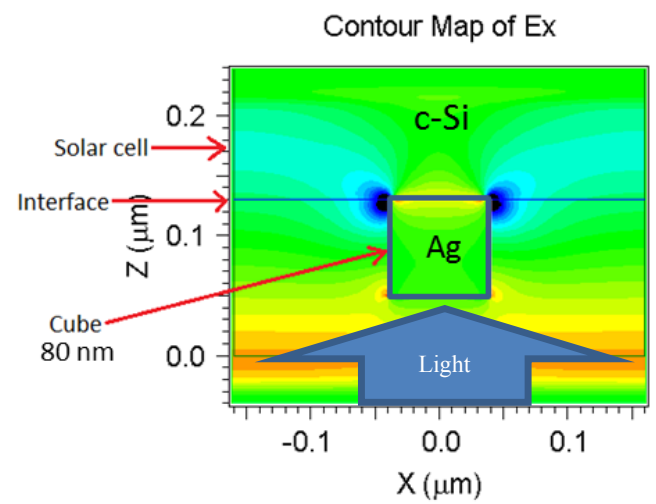

(a)

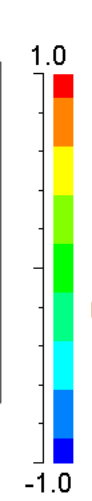

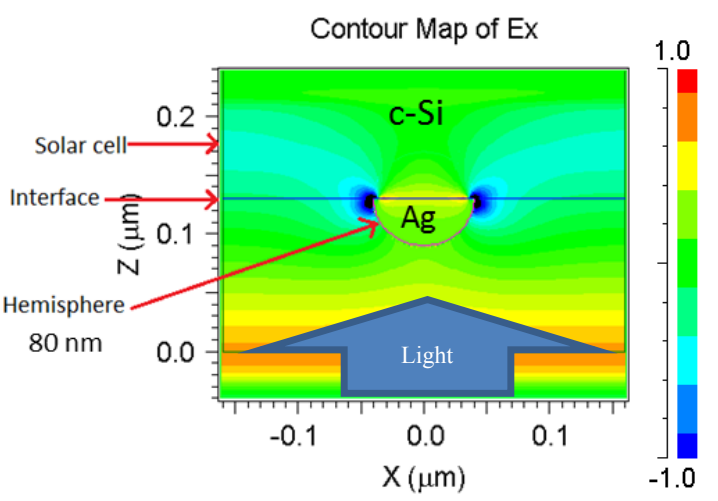

(b)

Figure 3-2: Electric field distribution of Ag MNP deposited on c-Si solar cell (a) cube with $80 \mathrm{~nm}$ in edge length and (b) hemisphere with $80 \mathrm{~nm}$ in diameter

The width of the cubic Ag MNPs in the simulation image above as well as the edge length diameter of the hemispherical Ag MNPs was $80 \mathrm{~nm}$. The area surrounding the MNPs was filled with air. The incident light comes from the bottom of the simulation through the solar cell and Ag MNP to the top. The electric field energy bar on the right side indicates intensity of electric field in the simulation. The solid line on the z-axis at zero was a launch pad in the simulator to provide incident light. The light intensity from the launch pad was set to one sum condition. Because the Ag MNP was close to the launch pad, an electric field was generated between the top of cubic Ag MNP and the launch pad. This electric field did not impact the electric field in the solar cell generated by the LSPR of Ag MNPs.

The dark blue and light blue color areas beside the Ag MNPs in the figure were the electric field caused by the scattering light. Based on the simulation results, the light scattering into the solar cell appeared from the corner of the cubic and hemispherical Ag MNPs. The difference of light scattering into the solar cell between the cubic and the hemispherical Ag MNPs was not distinct in the electric field distribution image. Based on two simulation images, both cubic and hemispherical Ag MNPs had the same amount of 
light scattering into the solar cell. The yellow color area at the interface between the nanoparticle and the solar cell was the backward electric field caused by the metal nanoparticle. In the cubic nanoparticle simulation image, the intensity of backward electric field at the interface was displayed as yellow and slightly red in color. However, the intensity of backward electric field between the hemispherical Ag MNPs and the solar cell presented as yellow only. We conclude that the cubic Ag MNPs deposited on the front surface of c-Si solar cell had higher backward electric field density compared to the Ag MNPs with other shapes.

\section{Effect on Efficiency Enhancement}

The effects of Ag MNP shape on efficiency enhancement of c-Si solar cells were investigated. The solar cell substrates used in this section were same as the solar cell substrates explained in the literature review chapter. The launch pad in the simulation is the light source, which has a light spectrum from $200 \mathrm{~nm}$ to $1200 \mathrm{~nm}$ wavelength with standard one sum light intensity. A c-Si solar cell with a p-n junction had a cell efficiency of $3.99 \%$ and $72 \%$ collective efficiency at this simulation system, which was same as the simulation result from the previous section. 


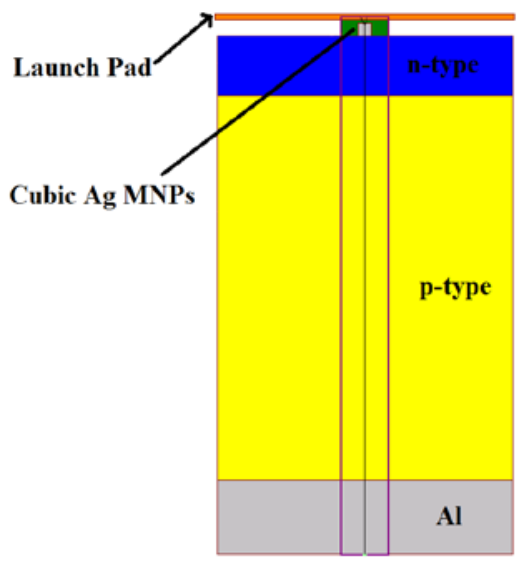

(a)

Solar Cell J-V Curve (Cell Efficiency $=3.87097 \%$ )

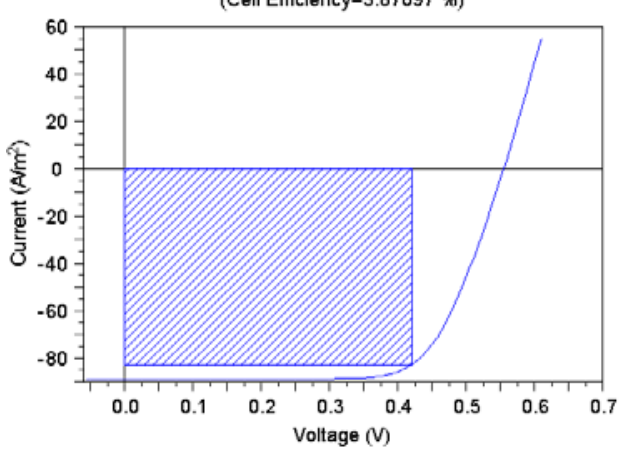

(c)

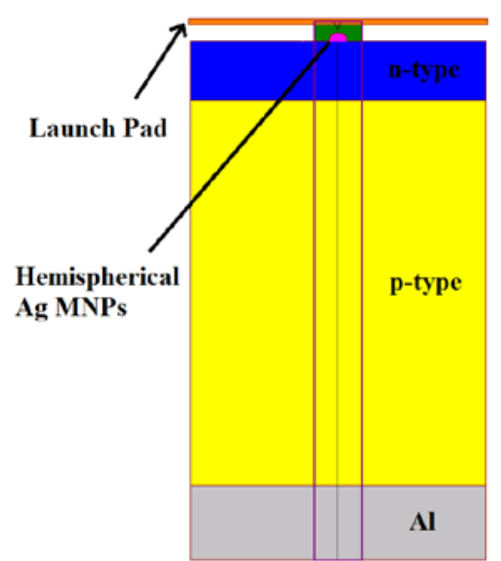

(b)

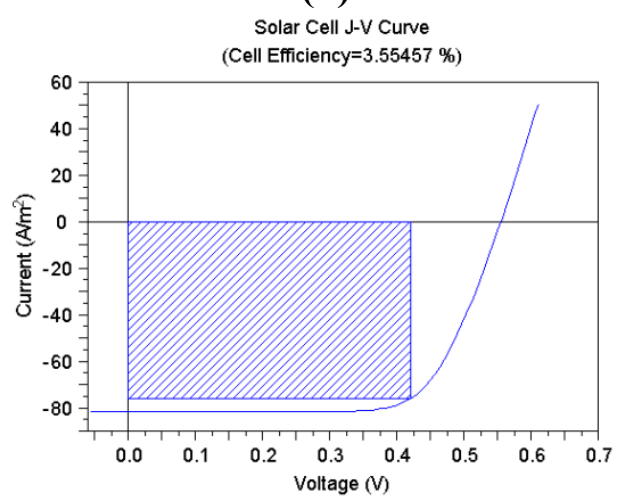

(d)

Figure 3-3: Schematic view of c-Si solar cell with (a) $80 \mathrm{~nm}$ size cubic Ag MNPs, and (b) $80 \mathrm{~nm}$ size hemispherical Ag MNPs. Simulation results for solar cell with (c) cubic Ag MNPs and (d) hemispherical Ag MNPs

Fig. 3-3 above, (a) shows a schematic view of the cubic Ag MNPs on c-Si solar cell, and (b) shows a schematic view of the hemispherical Ag MNPs on c-Si solar cell. Fig. 33 (c) shows the simulation results from the solar cell explained in Fig. 3-3 (a), while Fig. 3-3 (d) shows the simulation results from the solar cell explained in Fig. 3-3 (b). The MNP material used in this simulation was Ag. The size of cubic Ag MNPs was $80 \mathrm{~nm}$, while the diameter of hemispherical Ag MNPs was $80 \mathrm{~nm}$. The surface coverage on both solar cells is set to be $20 \%$. Based on the simulation results above, the c-Si solar cell with cubic Ag MNPs had a higher efficiency, 3.87\%, than the one with hemispherical Ag MNPs, which was 3.55\%. However, the c-Si solar cell without Ag MNPs on the top 
surface had 3.99\% under the same conditions, which was higher than the solar cell with cubic Ag MNPs. Deposited cubic Ag MNPs on the top of c-Si solar cell did not enhance the cell efficiency in the simulation system by following Ag MNPs parameters: 80nm edge length, $20 \%$ surface coverage, and no sublayer. Investigation of effects from Ag MNPs parameters on efficiency enhancement became important at this point in order to sufficiency apply Ag MNPs on c-Si solar cells.

\subsubsection{Effect of Ag MNPs Size on Efficiency Enhancement}

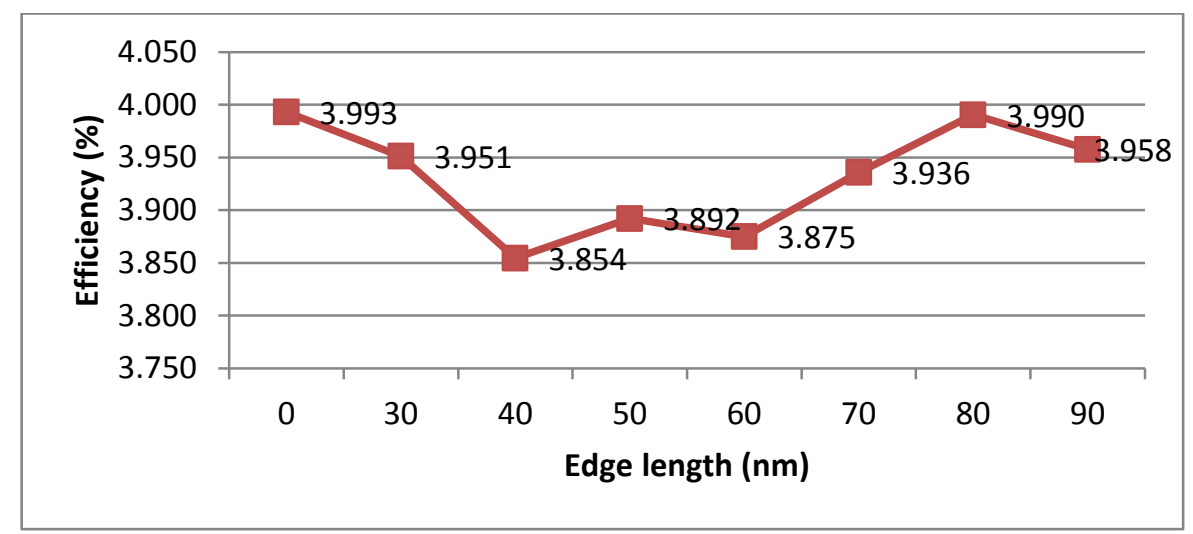

Figure 3-4: Cubic Ag MNPs on c-Si solar cell with different edge length

Fig. 3-4 above shows the effect of cubic Ag MNPs size on solar cell efficiency. Cubic Ag MNPs were deposited on the front of c-Si solar cells with 10\% surface coverage. As previously stated, the LSPR for each MNP is effect by particle size. According to the simulation results from previous section, cubic Ag MNPs have better efficiency enhancement on c-Si solar cells than hemispherical Ag MNPs. Investigation of Ag MNP size effect will focus on the cubic Ag MNPs only. Based on the data presented in Fig. 34, cubic Ag MNPs show the best performance when they have an edge length of approximately $80 \mathrm{~nm}$. These results disagree somewhat with some literature, which suggests that large Ag MNPs of approximately $100 \mathrm{~nm}$ should show the best 
enhancements for efficiency [46]. Their reasoning behind this is that larger MNPs have an LSPR that is further towards the red end of the visible spectrum and that higher-order modes will largely be attributed to the type of solar cell that we were simulating. Large MNPs, which have an LSPR closer to the infrared region, would show the greatest enhancement in absorption with light close to infrared region, which is a weak area of absorption for amorphous silicon (a-Si) solar cells. However, our simulation model was of c-Si, not a-Si solar cell, and for this reason, the strong near infrared enhancement of large MNPs may have been redundant and ineffective.

Another reason for the maximum efficiency occurred at $80 \mathrm{~nm}$ size Ag MNPs on c-Si solar cell was the redshift of resonance wavelength of Ag MNPs induced by the increased MNPs size. According to the literature, the resonance wavelength of MNP is related to the size of MNPs and shifts to a longer wavelength after the MNPs increase in size. The original reason the wavelength of Ag MNPs was set at $350 \mathrm{~nm}$ was to be out of the c-Si absorption spectrum. After increasing the size of Ag MNPs to $80 \mathrm{~nm}$ in edge length, the resonance wavelength shifted from $350 \mathrm{~nm}$ to $400 \mathrm{~nm}$ wavelength, which is in the c-Si absorption spectrum. However, the absorption coefficient of c-Si solar cells decreased rapid after $400 \mathrm{~nm}$ wavelengths, which made further shifting the resonance wavelength less valuable to the MNP design. We concluded that the optimal size of cubic Ag MNPs was $80 \mathrm{~nm}$ in edge length. 


\subsubsection{Effect of Ag MNPs Surrounding Media on Plasmonics and Efficiency Enhancement}

The refractive index of the surrounding medium was important for the light scattering ability of MNPs. By using a low refractive index medium as a sublayer between the MNPs and the solar cell, the light scattering ability of MNPs was increased. More light scattered into solar cell caused increased electric field intensity under the MNPs. F. J. Beck demonstrates an effective method of enhancing light trapping in solar cells with a thin sublayer between the array of Ag MNPs and the solar cell substrate [47]. They also investigated the effects of $\mathrm{SiO}_{2}, \mathrm{Si}_{3} \mathrm{~N}_{4}$, and $\mathrm{TiO}_{2}$ as the medium of sublayer between the $\mathrm{Ag}$ MNPs and the solar cell. Their experimental results show that the $\mathrm{SiO}_{2}$ sublayer had the best enhancement across the three mediums [47]. In this section, the effect of deposited cubic and hemispherical Ag MNPs on the $\mathrm{SiO}_{2}$ sublayer of the top solar cell surface was investigated.

\section{Effect on Plasmonic Enhancement}

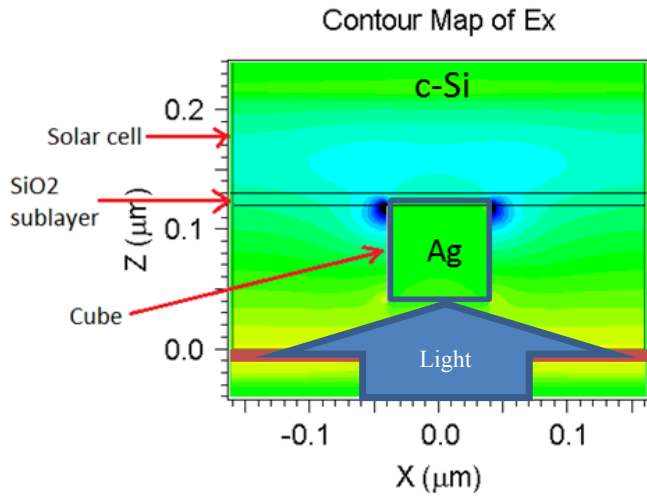

(a)

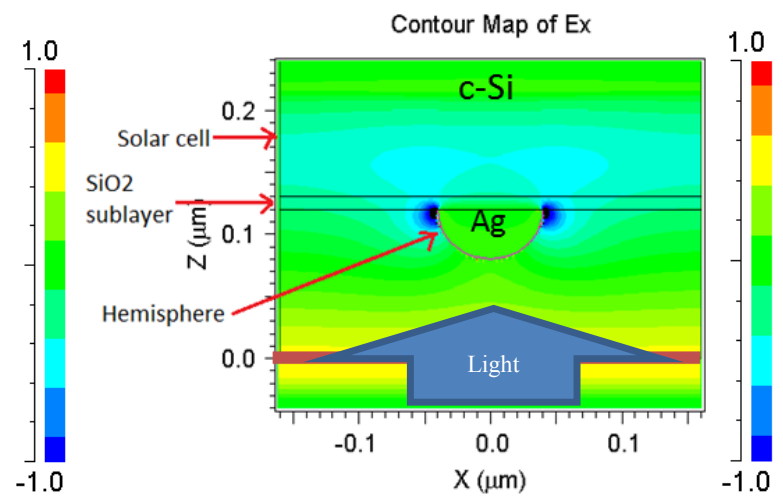

(b)

Figure 3-5: Electric field distribution of Ag MNP set on $10 \mathrm{~nm}$ thick $\mathrm{SiO}_{2}$ sublayer (a) $80 \mathrm{~nm}$ size cube and (b) $80 \mathrm{~nm}$ size hemisphere

Fig. 3-5 (a) displays the electric field distribution of a cubic Ag MNP deposited on a $\mathrm{SiO}_{2}$ sublayer on the front surface of the solar cell, while Fig. 3-5 (b) shows the electric 
field distribution of a hemispherical Ag MNP deposited on a $\mathrm{SiO}_{2}$ sublayer on the front surface of the solar cell. The thickness of the $\mathrm{SiO}_{2}$ sublayer in both simulation result images was $10 \mathrm{~nm}$ because it is an optimized thickness for Ag MNPs with $80 \mathrm{~nm}$ diameter [12]. The size of the cubic Ag MNP was $80 \mathrm{~nm}$ as was the diameter of the hemispherical Ag MNPs.

According to the simulation results from the two types of Ag MNPs, embedding a dielectrical sublayer between the Ag MNPs and the solar cell was removed to enhance the forward electric field between the Ag MNPs and the solar cell. No backward electric field was observed in the simulation results of the cubic Ag MNPs and the hemispherical Ag MNPs. The light blue color area in the solar cell of both simulation diagrams was the increased electric field intensity due to the LSPR from MNPs. The electric field from the cubic Ag MNPs had bigger dark blue areas in the simulation image compared to the electric field of the hemispherical Ag MNPs simulation image. This dark blue region was the enhanced electric field in the solar cell induced by the forward electric field from the MNPs. After adding a low refractive index material between the MNPs and the solar cell, the electric field originally existed at the corner of cubic MNPs was distributed into the solar cell. The released electric field from the corner increases the area of electric field in the solar cell. Due to higher electric field intensity driving into the solar cell caused by LSPR, the solar cell with cubic Ag MNPs on the $\mathrm{SiO}_{2}$ sublayer had higher cell efficiency compared to the solar cell with the hemispherical Ag MNPs on the $\mathrm{SiO}_{2}$ sublayer. The next simulation step was to simulate the solar cell with Ag MNPs through the solar cell utility. 


\section{Effect on Efficiency Enhancement}

The enhanced forward electrical to enhance the cell efficiency of Ag MNPs is exploited for the design of a sublayer that enhances the forward electric field generated by Ag MNPs. Ag MNPs with $80 \mathrm{~nm}$ in edge length are chosen for their high scattering efficiency and advantage in fabrication procedures. The Ag MNPs are formed directly on top of two different dielectrics commonly used in silicon-based solar cell investigation: $\mathrm{SiO}_{2}$ and indium tin oxide (ITO), with refractive indices of 1.5 and 2.35 , respectively [48]. There are other dielectrical materials that can be used as the material for sublayers, like $\mathrm{Si}_{3} \mathrm{~N}_{4}$ and $\mathrm{TiO}_{2}$.

In order to choose an optimal sublayer with maximum enhancement, the refractive index of sublayer material has to be low so it will not reduce the LSPR generated by the Ag MNPs. The $\mathrm{SiO}_{2}$ has the lowest refractive index, which makes it the optimal sublayer material. The ITO is a conductive material that is not suitable to apply on the c-Si front surface since it will short-circuit the front conductor. The refractive index of $\mathrm{Si}_{3} \mathrm{~N}_{4}$ and $\mathrm{TiO}_{2}$ are 2.0 and 2.5, which are higher than the refractive index of $\mathrm{SiO}_{2}$, rendering them unsuitable for finding the optimal sublayer material. Hence, we conclude that the $\mathrm{SiO}_{2}$ are better compared to other sublayer materials. 

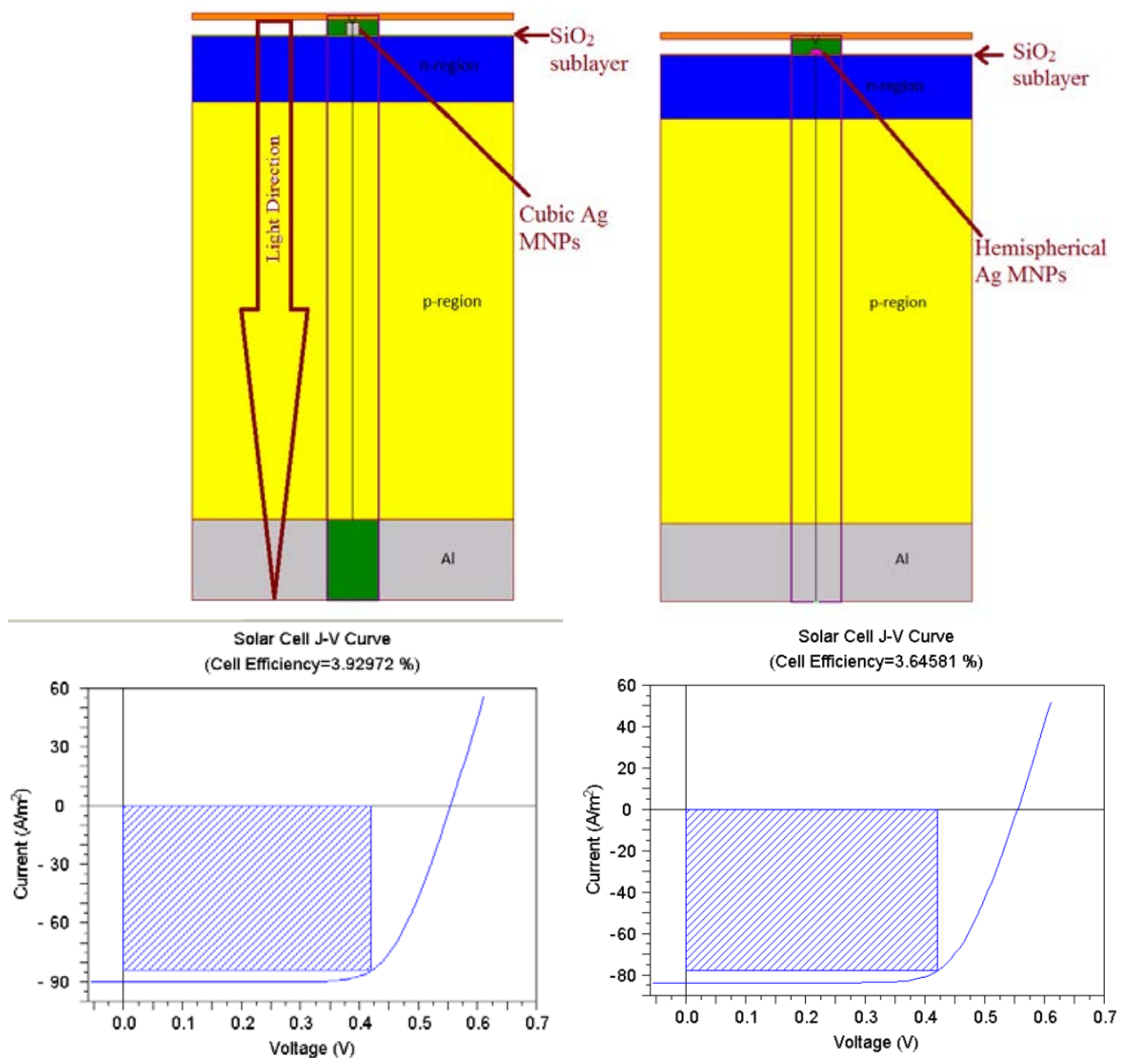

Figure 3-6: Schematic view of $10 \mathrm{~nm}$ thick $\mathrm{SiO}_{2}$ sublayer on c-Si solar cell with (a) $80 \mathrm{~nm}$ size cubic Ag MNPs, and (b) $80 \mathrm{~nm}$ size hemispherical Ag MNPs. Simulation results for solar cell with (c) cubic Ag MNPs and (d) hemispherical Ag MNPs

Fig. 3-6 (a) shows a schematic view of the $80 \mathrm{~nm}$ size cubic Ag MNPs with 20\% surface coverage on the c-Si solar cell, and (b) shows a schematic view of the $80 \mathrm{~nm}$ size hemispherical Ag MNPs with 20\% surface coverage on the c-Si solar cell. Fig. 3-6 (c) shows the simulation results from the solar cell explained in Fig. 3-6 (a), while Fig. 3-6 (d) shows the simulation results from the solar cell explained in Fig. 3-6 (b). Based on the simulation results above, the c-Si solar cell with cubic Ag MNPs had a higher efficiency, $3.92 \%$, than when employing hemispherical Ag MNPs, which was 3.64\%. Comparing to the cubic Ag MNPs solar cell without a sublayer, inserting a sublayer between the MNPs and the solar cell increases the cell efficiency by $1.3 \%$. This increased efficiency was 
caused by the increased electric field area in the solar cell. However, the original c-Si solar cell had $3.99 \%$ cell efficiency, which was higher than the solar cell with cubic Ag MNPs. Deposited cubic Ag MNPs on the top of c-Si solar cell had negative enhancement on the cell efficiency in the simulation system with the following Ag MNPs parameters: $80 \mathrm{~nm}$ in edge length, 20\% surface coverage, and $10 \mathrm{~nm}$ thick $\mathrm{SiO}_{2}$ sublayer. The optimal size of Ag MNPs was $80 \mathrm{~nm}$ in edge length since the resonance wavelength of $\mathrm{Ag}$ at this size had best much with the absorption coefficient of the c-Si solar cell. Investigation of effects on the surface coverage and the sublayer thickness on efficiency enhancement became important at this point in order to optimize efficiency enhancement.

We conclude that the $\mathrm{Ag}$ MNPs with a $\mathrm{SiO}_{2}$ sublayer are better compared to the $\mathrm{Ag}$ MNPs directly deposited on c-Si solar cell because the $\mathrm{SiO}_{2}$ sublayer enhances the forward electric field from Ag MNPs.

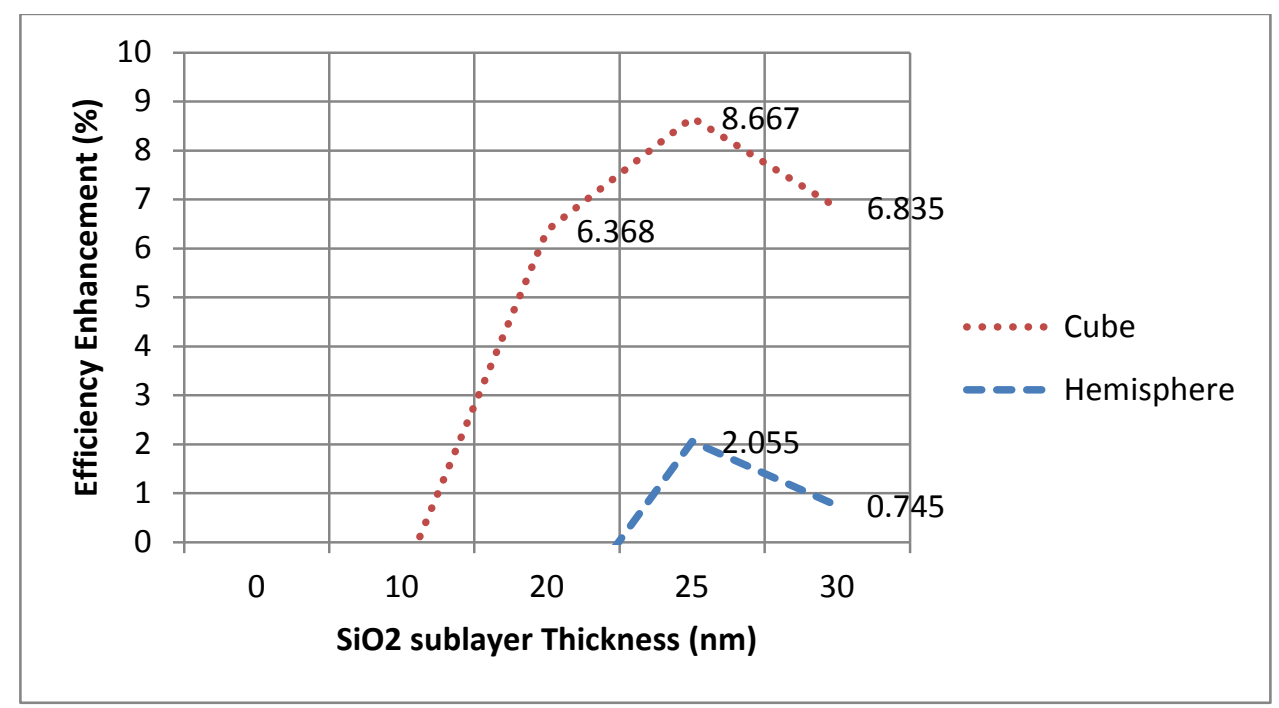

Figure 3-7: Efficiency enhancement by $80 \mathrm{~nm}$ size cubic Ag MNPs and hemispherical Ag MNPs with $20 \%$ surface coverage on different thickness $\mathrm{SiO}_{2}$ sublayer

Based on the simulation results in the previous section, deposited cubic and hemispherical Ag MNPs on the $\mathrm{SiO}_{2}$ sublayer enhanced the forward electric field from the LSPR due to decrease refractive index on surrounding media. Fig. 3-7 shows the cell 
efficiency enhancement from $80 \mathrm{~nm}$ size cubic Ag MNPs and hemispherical Ag MNPs deposited on the $\mathrm{SiO}_{2}$ sublayer with different thicknesses. The Ag MNP surface coverage used in this simulation was $20 \%$ for both cubic and hemispherical Ag MNPs. According to the simulation results, the cell efficiency enhancement from cubic Ag MNPs was higher than that from hemispherical $\mathrm{Ag}$ MNPs on the $\mathrm{SiO}_{2}$ sublayer at all thicknesses. The solar cell with cubic Ag MNPs deposited on the $25 \mathrm{~nm}$ thick $\mathrm{SiO}_{2}$ sublayer had the highest cell efficiency enhancement, which was $8.667 \%$. This was due to the enhanced forward electric field from the LSPR of cubic Ag MNPs with the $\mathrm{SiO}_{2}$ sublayer. For c-Si solar cells, electric field enhancement underneath the Ag MNPs was the main feature of Ag MNPs deposited on the top surface. With the $\mathrm{SiO}_{2}$ sublayer between the MNPs and the solar cell, cubic Ag MNPs gained higher efficiency enhancement from the dielectrical sublayer than hemispherical Ag MNPs. We conclude that the cubic Ag MNPs on the sublayer have better efficiency enhancement compared to the hemispherical Ag MNPs. 


\subsubsection{Effect of Ag MNPs Surface Coverage on Solar Cell efficiency}

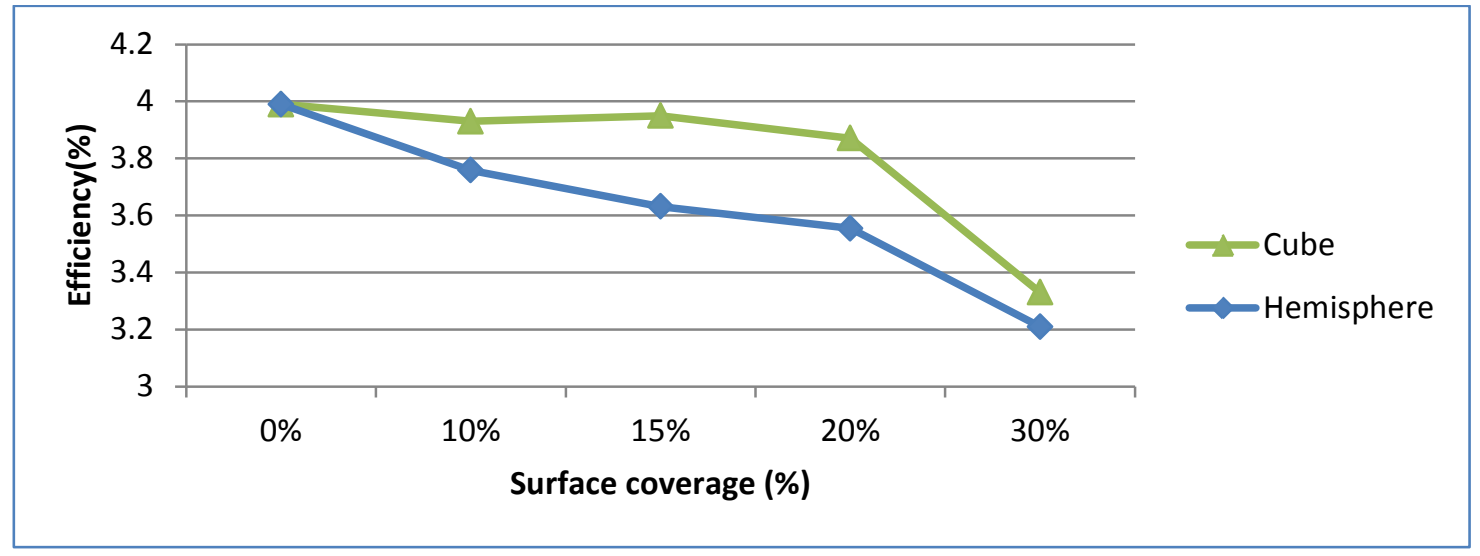

Figure 3-8: $80 \mathrm{~nm}$ size cubic Ag MNPs and $80 \mathrm{~nm}$ size hemispherical Ag MNPs deposited on the c-Si solar cell with different surface coverages

The surface coverage of nanoparticles on the solar cell was the density of Ag MNPs distributed on the top of the solar cell or the sublayer. High surface coverage means more nanoparticles were deposited on the solar cell surface. The blue curve in Fig. 3-8 is the cell efficiency plotting for the solar cell with hemispherical Ag MNPs deposited on the top surface with different surface coverage levels. The green curve in Fig. 3-8 is the cell efficiency for the solar cell with cubic Ag MNPs deposited on the top surface with different surface coverage levels. The cubic Ag MNPs were $80 \mathrm{~nm}$ in edge length, while the hemispherical Ag MNPs were $80 \mathrm{~nm}$ in diameter.

Based on the simulation results, deposited cubic Ag MNPs on the top of the c-Si solar cell did not enhance the cell efficiency at all surface coverages. When cubic Ag MNPs surface coverage was $10 \%$, the cell efficiency of the solar cell with cubic Ag MNPs on the top decreased about 10\% compared to the solar cell without cubic Ag MNPs because increased efficiency caused by the scattering light from Ag MNPs was less than decreased efficiency caused by reduced incident light from Ag MNPs. Deposited Ag MNPs on the front of solar cell will generate plasmonic resonance around along with 
shading losses at the bottom. The shading losses at the bottom will reduce incident light on the solar cell, which decreases efficiency. The efficiency enhancement from MNPs occurred when the increased efficiency from the plasmonic resonance was larger than the decreased efficiency from the shading losses. Based on the simulation results, increasing the surface coverage of cubic Ag MNPs on the c-Si solar cell further decreased cell efficiency. The simulation result matched the result from the literature review section that deposited cubic Ag MNPs on the c-Si solar cell without the sublayer were not enhanced efficiency.

According to the simulation results, deposited the hemispherical Ag MNPs on the solar cell did not enhance the cell efficiency at all surface coverages. The efficiency enhancement caused by plasmonic resonance from hemispherical Ag MNPs was less than that for cubic Ag MNPs when Ag MNPs were directly deposited on the c-Si solar cell front surface and was surrounded with air. However, the Ag MNP surface coverage simulation results proved that directly deposited cubic or hemispherical Ag MNPs on c-Si solar cell generated negative efficiency enhancement at any surface coverage condition. We conclude that the cubic Ag MNPs have better efficiency enhancement compared to hemispherical Ag MNPs when they were directly deposited on the c-Si solar cell front surface. 


\subsubsection{MNP Optimization}

Many factors determine how effective an array of plasmonic Ag MNPs is in enhancing light absorption and photocurrent generation in the c-Si solar cell. The resonance wavelength of MNPs is affected by their size and shape. The refractive index of MNPs surrounding medium can also affect the LSPR occurring on them. Varying any of these parameters allows large changes in LSPR behaviors and the electrical properties of solar cells after MNPs are applied to it. After many investigations on the working function of MNPs with or without solar cells, an optimization of MNP performance on solar cells in terms of all of the parameters from simulation has to be discussed before beginning experiments and actually fabricating an MNP enhanced c-Si solar cell.

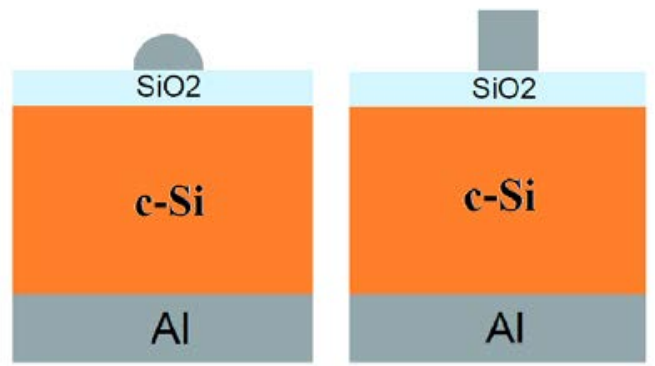

Figure 3-9: Simulated geometry for c-Si solar cells with Al back contact, c-Si absorber, and $\mathrm{SiO}_{2}$ sublayer for (a) hemispherical Ag MNPs and (b) cubic Ag MNPs

Fig. 3-9 above shows the simulated geometry for c-Si solar cells with hemispherical and cubic Ag MNPs on the front surface. Optimizations were performed for $80 \mathrm{~nm}$ diameter hemispherical Ag MNPs with an $80 \mathrm{~nm}$ diameter and cubic Ag MNPs with an edge length of $80 \mathrm{~nm}$ on the $\mathrm{SiO}_{2}$ sublayer so that relevant comparisons of enhancements could be made for the most common shapes and plasmonic materials. The optimizations were explained by using three-dimensional trends in values across two dimensions in a continuous cover, which are surface coverage and $\mathrm{SiO}_{2}$ sublayer thickness. In the plot, 
the vertical axis in the plot displays the enhancement of cell efficiency. The horizontal axis displays the thickness of the $\mathrm{SiO}_{2}$ sublayer. The depth axis displays the surface coverage of Ag MNPs. The color-enhancement bar on the top of the graph shows defined color for the cell efficiency enhancement at different levels.

The efficiency enhancement is calculated by comparing the solar cell with hemispherical Ag MNPs and the one without hemispherical Ag MNPs, with both cells containing the $\mathrm{SiO}_{2}$ sublayer between the c-Si solar cell and the MNPs. Therefore, the optimization plots show the cell efficiency enhancement from the hemispherical Ag MNPs only. For example, the c-Si solar cell with $80 \mathrm{~nm}$ hemispherical Ag MNPs and with $10 \%$ surface coverage deposited on a $10 \mathrm{~nm}$ thick $\mathrm{SiO}_{2}$ layer has a cell efficiency equal to $3.8 \%$. The c-Si solar cell with the $10 \mathrm{~nm}$ thick $\mathrm{SiO}_{2}$ layer on the front surface has a cell efficiency equal to $4.0 \%$, which is lower than the cell efficiency from the pure c-Si solar cell. Comparison between the solar cell with $10 \%$ surface coverage for hemispherical Ag MNPs and the solar cell without hemispherical Ag MNPs shows that the cell efficiency for the solar cell with $10 \%$ surface coverage decreased $5.1 \%$ compared to the original cell efficiency. Hence, the hemispherical Ag MNPs on the c-Si solar cell did not enhance cell efficiency when surface coverage equaled $10 \%$ and the $\mathrm{SiO}_{2}$ sublayer thickness equaled $10 \mathrm{~nm}$. The same methods will be used to optimize cubic $\mathrm{Ag}$ MNPs on the c-Si solar cell. 


\section{Optimization of Hemispherical Ag MNPs}

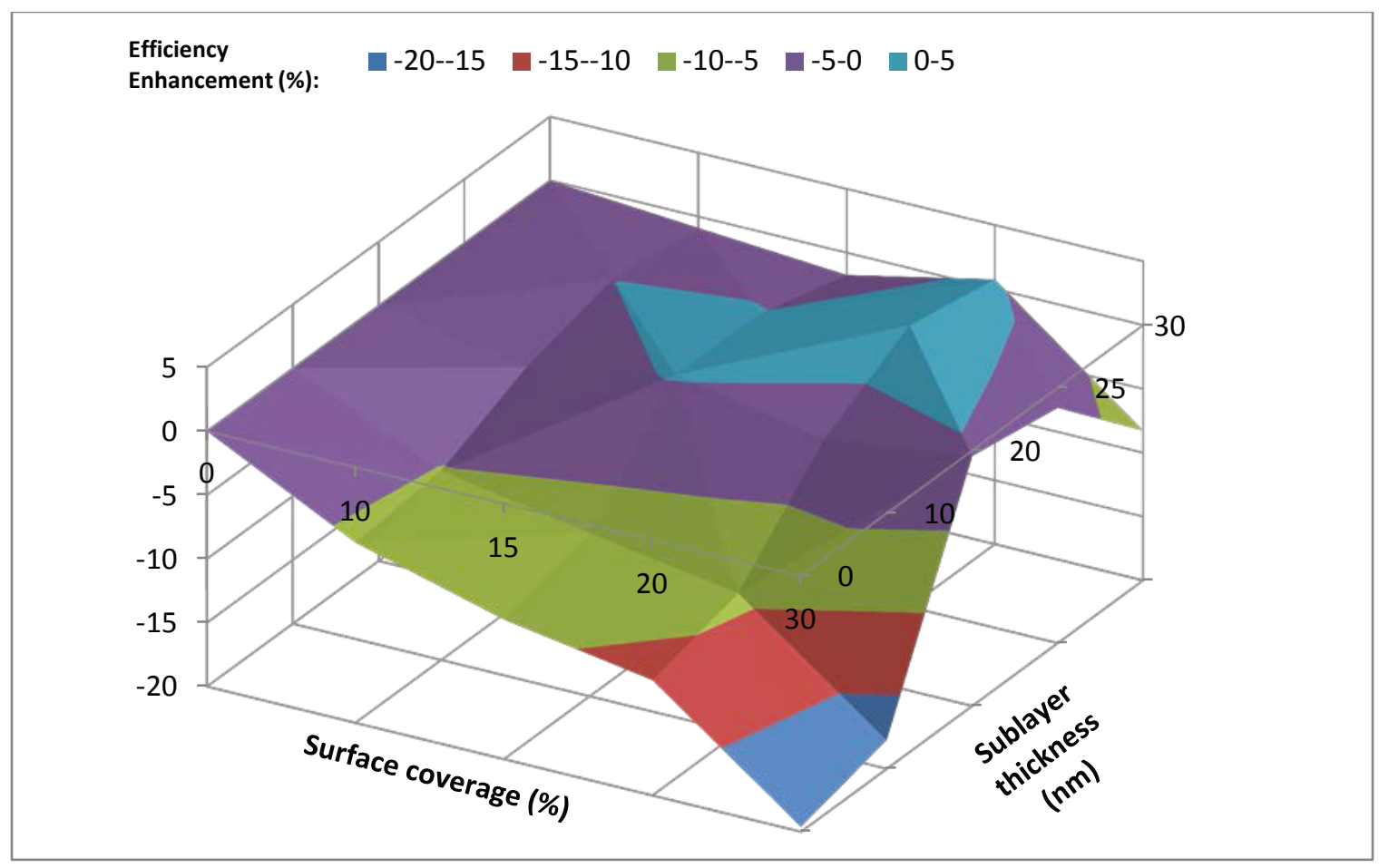

Figure 3-10: Optimized efficiency enhancement of 80nm size hemispherical Ag MNPs with different surface coverages and $\mathrm{SiO}_{2}$ sublayer thicknesses

Fig. 3-10 above shows the simulation results of $80 \mathrm{~nm}$ size hemispherical Ag MNPs deposited on the c-Si solar cell at different surface coverage levels with and without the $\mathrm{SiO}_{2}$ sublayer. The thickness of the $\mathrm{SiO}_{2}$ sublayer changes from $0 \mathrm{~nm}$ to $30 \mathrm{~nm}$. The optimal cell efficiency enhancement for the $80 \mathrm{~nm}$ edge length hemispherical Ag MNPs deposited on the c-Si solar cell was $2.05 \%$ when surface coverage of Ag hemispherical NPs was $20 \%$ and the thickness of the $\mathrm{SiO}_{2}$ sublayer was $25 \mathrm{~nm}$. The negative efficiency enhancement in the simulation means a decreased cell efficiency caused by the hemispherical Ag MNPs.

The overall cell efficiency enhancement for hemispherical Ag MNPs was not as good as it was explained in the literature [12]. Only two cell efficiency enhancements occurred when hemispherical Ag MNPs were deposited on the c-Si solar cell, which was 
hemispherical Ag MNPs with 15\% and 20\% surface coverage deposited on $25 \mathrm{~nm}$ and 30 $n m$ thicker $\mathrm{SiO}_{2}$ sublayers. When the $\mathrm{SiO}_{2}$ sublayer thickness equals $0 \mathrm{~nm}$, the hemispherical Ag MNPs were deposited on the c-Si solar cell without the $\mathrm{SiO}_{2}$ sublayer between the solar cell and the MNPs. The simulation results show that hemispherical Ag MNPs deposited directly on the c-Si solar cell were decreasing cell efficiency from $5 \%$ to $19 \%$ depending on the surface coverage of hemispherical Ag MNPs. However, simulation results from the literatures show that hemispherical Ag MNPs deposited on the a-Si solar cell enhanced cell efficiency [24]. Due to difference on optical properties and substrate thicknesses between the c-Si and the a-Si solar cells, the working function of hemispherical Ag MNPs can be different on these two types of solar cells.

The efficiency enhancement of $80 \mathrm{~nm}$ size hemispherical Ag MNPs was decreased when the sublayer thickness equaled $30 \mathrm{~nm}$. The reason of this is that increasing the thickness of the sublayer decreases the coupling efficiency as the particle is moved further away from the silicon substrate. And increasing the thickness of the sublayer will also increase the blue shifting of the resonance wavelength. Additionally, increasing the thickness of the sublayer increases the amount of scattering light into the substrate [49]. There is a trade-off between the benefits of enhanced the forward electric field and the reductions in the coupling efficiency as the sublayer gets thicker.

We conclude that the optimal cell efficiency enhancement from hemispherical Ag MNPs deposited on the c-Si solar cell is $2 \%$, in which the surface coverage of the hemispherical Ag MNPs is $20 \%$ and the $\mathrm{SiO}_{2}$ sublayer thickness is $25 \mathrm{~nm}$. 


\section{Optimization of Cubic Ag MNPs}

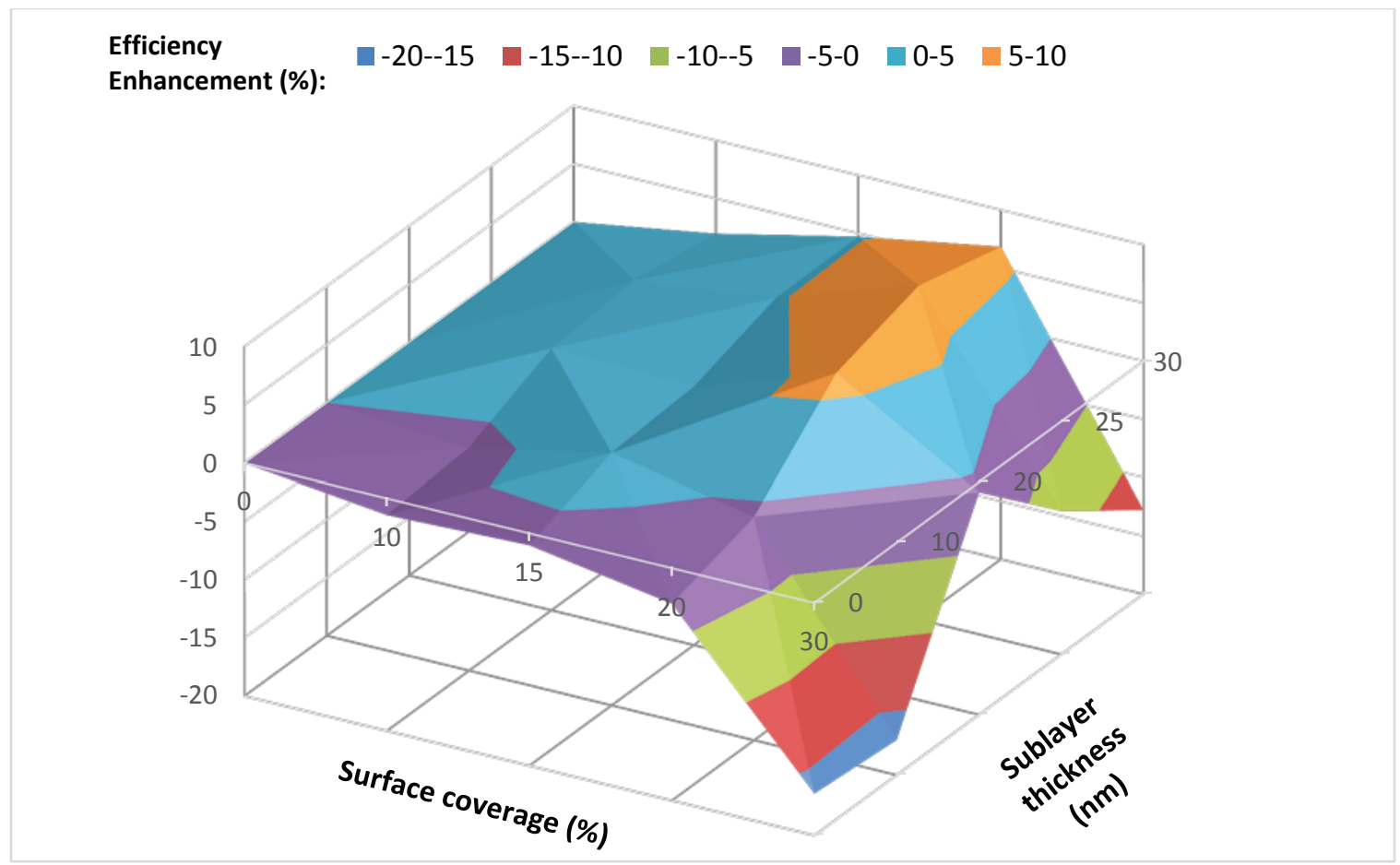

Figure 3-11: Optimized efficiency enhancement of $80 \mathrm{~nm}$ size cubic Ag MNPs with different surface coverages and $\mathrm{SiO}_{2}$ sublayer thicknesses

The next optimization was performed for $80 \mathrm{~nm}$ size cubic Ag MNPs on the c-Si solar cell with and without the $\mathrm{SiO}_{2}$ sublayer, and Fig. 3-11 above show the optimized efficiency enhancement plot. The optimal cell efficiency enhancement caused by the 80 $n m$ cubic Ag MNPs was 8\% when the surface coverage of cubic Ag MNPs was 20\% and the thickness of the $\mathrm{SiO}_{2}$ sublayer was $20 \mathrm{~nm}$. This cell efficiency enhancement was larger than the cell efficiency enhancement from the hemispherical Ag MNPs, which was $2 \%$.

Cubic Ag MNPs applied to the c-Si solar cell did not always enhance cell efficiency; cell efficiency decreased when the cubic Ag MNPs were deposited on the pure c-Si solar cell without the $\mathrm{SiO}_{2}$ sublayer. Similar to the hemispherical Ag MNPs deposited on the pure c-Si solar cell, higher surface coverage of the cubic Ag MNPs caused a larger 
decrease in cell efficiency. The solar cell did not receive augmented optical properties from the cubic Ag MNPs and their scattering ability of incident light. On the other hand, those cubic Ag MNPs deposited on the front surface reduced the amount of light that reached the solar cell, which caused fewer photons to absorb in the solar cell, thereby decreasing its efficiency, known as shading losses.

After insetting the $\mathrm{SiO}_{2}$ sublayer between the c-Si solar cell and the cubic Ag MNPs, the cubic Ag MNPs started to enhance the cell efficiency of the c-Si solar cell. When the sublayer was $10 \mathrm{~nm}$ thicker, cell efficiency enhancement from the cubic Ag MNPs only occurred at a surface coverage equal to $15 \%$. The scattering ability from the cubic $\mathrm{Ag}$ MNPs was not strong enough to balance the shading losses from the cubic Ag MNPs in terms of cell efficiency. As the thickness of the $\mathrm{SiO}_{2}$ sublayer kept increasing, the cubic Ag MNPs were able to enhance cell efficiency when the surface coverage of MNPs was $10 \%, 15 \%$, or $20 \%$. Peak cell efficiency enhancement occurring on the surface coverage of cubic Ag MNPs equaled 20\%. Based on the simulation results, cell efficiencies for the solar cell with 30\% surface coverage cubic Ag MNPs was lower than the solar cell without cubic Ag MNPs in any conditions, with or without the sublayer. The reason for this situation was caused by the lack of scattering ability from the cubic Ag MNPs. The enhanced efficiency caused by scattering ability was less than the decreased efficiency caused by the shading losses, in which total cell efficiency was decreasing.

As a conclusion, the optimal cell efficiency enhancement caused by Ag MNPs was done by cubic Ag MNPs. Deposited $80 \mathrm{~nm}$ in edge length cubic Ag MNPs on the c-Si solar cell had $4.53 \%$ efficiency and a $8 \%$ efficiency enhancement compared to the 
original solar cell, when the surface coverage of the MNPs was $20 \%$ and the thickness of the $\mathrm{SiO}_{2}$ sublayer was $25 \mathrm{~nm}$.

\subsection{Surface Textured Nanostructures}

\subsubsection{Black Silicon Solar Cell}

An array of vertically aligned needle-shaped silicon structures can reduce the volume of specular reflectivity necessary for high efficiency by providing nearly ideal diffused reflectivity across the c-Si solar spectrum. Near-zero specular reflectivity over a broad range of incident angles is discussed by forming a needle-shaped layer with features close or equal to the wavelength of light, which eliminates the need for conventional surface textured structures and antireflection coating [34]. When the size of the surface features are smaller than the wavelength of incident light, needle-shaped nano- and microstructures also provide beneficial light trapping that increases the effective path length in the c-Si solar cell [50]. The size of b-Si structures is dependent on the geometry of the structure, and it will affect the solar cell reflectivity directly.
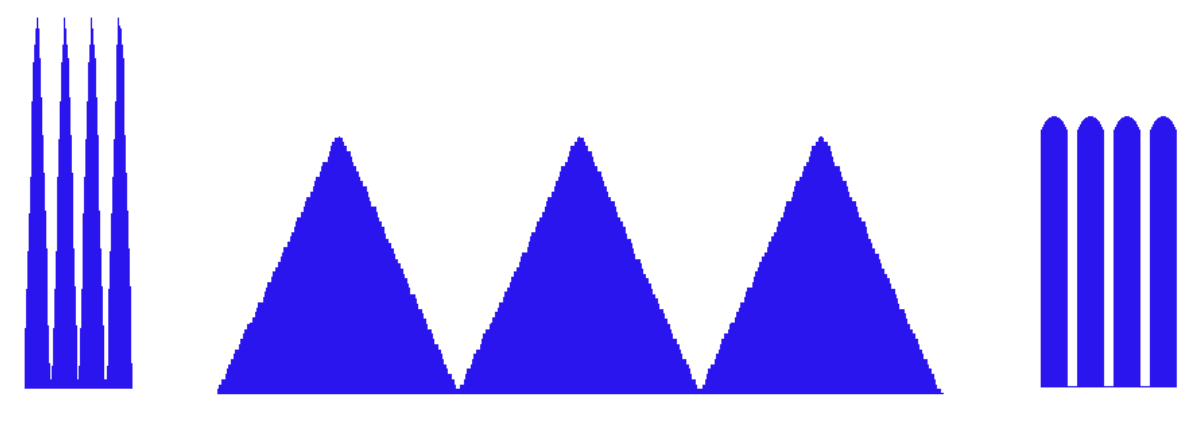

Figure 3-12: Needle-like shape, pyramid shape, and cylinder shape silicon structures

Fig. 3-12 above shows three basic b-Si structure shapes for solar cell application, needle-like, pyramid, and cylinder shaped. Silicon nanostructures on b-Si solar cells can be etched into different shapes as long as the reflectivity of visible light remains at a very 
low level. Three b-Si structures shown in the figure above are uniformly distributed on the solar cell surface; however, b-Si structures in experiments are usually distributed randomly over the silicon surface for most etching methods [29]. The pyramid-shaped silicon structures are usually fabricated at the micron scale. The needle-shaped and the cylinder-shaped structures on b-Si solar cells are fabricated at the nanometer scale. Based on the geometry of the three silicon structure shapes, the aspect ratios of the pyramidshaped structures are lower than the needle- and cylinder-shaped structures.

The pyramid-shaped silicon structures are the most commonly used surface-textured structures for solar cell applications. Silicon structures fabricated with this pyramid shape have the lowest fabrication cost among the three basic shapes. However, due to the scale and geometry of pyramid structures, silicon structures with a pyramid shape have the largest surface reflectivity of the three basic shapes. The cylinder-shaped silicon structures have the highest fabrication requirements. Each cylinder shaped structure has to be etched with high accuracy to form perpendicular side walls. The advantage of using cylinder-shaped structures is that they can be designed to reflect incident light at specific wavelengths. Since the cylinder-shaped structures only reflect light at specific wavelengths, they can be used to reduce total reflectivity of solar cells. However, the perpendicular side wall on cylinder structures increases reflectivity between cylinder structures, and the flat space between each cylinder structure causes high specular reflectivity. Hence, the solar cell with cylinder-shaped structures has greater surface reflectivity than the solar cell with needle-shaped structures. The solar cell with needleshaped structures has the lowest total reflectivity by comparison. The fabrication requirement for the needle-shaped structures is higher than that for the pyramid-shaped 
structures but lower than that for the cylinder-shaped structures. Since the most important feature of $b$-Si structures is their ability to reduce total reflectivity of solar cells, the b-Si structures herein are needle-shaped to achieve the lowest total reflectivity.

The shape of b-Si nanostructures is very important for b-Si solar cells, since it affects many features. The etching method used to fabricate b-Si solar cells determines the shape of $b$-Si structures after etching processes, where different etching methods generate different silicon nanostructure shapes. The needle- and cylinder shaped structures are commonly etched by the dry etching method due to their small scale. The pyramidshaped structures are of a larger scale than the other two shapes and are mostly fabricated by using the wet etching method. Another difference between each silicon nanostructure shape is the aspect ratio, where needle-shaped and cylinder-shaped nanostructures via the dry etching method have large aspect ratios compared to nanostructures with a pyramid shape. The wet etching methods have the disadvantage of etching perpendicular side walls and structures on a small scale, since most of them use chemical material to etch substrate. The silicon nanostructures that are needle-shaped and cylinder-shaped need to be etched by the dry etching method because both structures are in the nanometer scale and possess larger aspect ratios up to 10:1, with needle-like shaped structured having the lowest reflectivity [29].

C-Si solar cells based on b-Si structures exhibit lower power conversion efficiencies than high efficiency solar cells due to the increased photocarrier surface recombination effect. The needle-shaped structures give the lowest total reflectivity but also the largest surface area. Since the absorbed light spectrum of c-Si solar cells is between $400 \mathrm{~nm}$ and $1100 \mathrm{~nm}$, the height and width of needle-shaped structures should be in the range from 
$400 \mathrm{~nm}$ to $1100 \mathrm{~nm}$ to obtain the lowest reflectivity along with the smallest surface area. Because of the absorption spectrum of c-Si and the surface area requirement, the minimum size used in the structure is $400 \mathrm{~nm}$, and the maximum size used in the structure is $1100 \mathrm{~nm}$. It is well-known that the structure with small geometry generates a larger surface area compared to the structure with large geometry; so, needle-shaped structures smaller than $400 \mathrm{~nm}$ would increase surface area, which reduces cell efficiency for b-Si solar cells. Hence, the optimal b-Si solar cell contains needle-shaped structures (dimensions: $400 \mathrm{~nm}$ in width and $1100 \mathrm{~nm}$ in height) uniformly distributed over the c-Si solar cell surface, and the aspect ratio for optimally designed b-Si structures is 2.75 .

\subsubsection{Characterization Matrix}

The characterization of $\mathrm{b}-\mathrm{Si}$ solar cells can be separated into two parts, geometric analysis and electrical analysis. A scanning electron microscope (SEM) system was used to study the geometric of b-Si structures and verify defective structures on the b-Si solar cell. The SEM system was carried on by the Carleton University Department of Chemistry. The electrical part of the b-Si solar cell will be investigated by a photoluminescent (PL) measurement system at the University of Ottawa. The PL phenomenon is light emission from any form of material after the absorption of photons. Characterization techniques based on the PL phenomenon have recently emerged as powerful tools for developing and measuring optical and electrical properties of silicon based solar cells. Based on the PL measurement results, the electrical properties of the bSi solar cell can be determined before forming a complete solar cell.

PL techniques are broadly applicable to silicon solar cell development, give optical perception into the operation of substrate, and identify how substrate performance can be 
improved in a short time period [51]. Laser-based PL techniques have been widely used, at room temperature and cryogenic temperatures, to investigate dopant and defective properties in silicon and to measure the recombination effect on carrier lifetime inside the operation of the substrate [52][53].

Two PL technologies are frequently used to measure and analyze solar cell performance, the PL imaging method and the PL intensity numerical analysis method. PL imaging is a technique for fast characterization of silicon solar cells by revealing a host of substrate properties. In the PL imaging technique, the entire silicon solar cell surface is illuminated homogeneously under a high power fiber-coupled infrared (IR) laser source. A sensitive IR camera captures the external optical excitation and stimulates PL signal emitted from the sample surface. Because of the characterization of silicon solar cells by PL is very sensitive at small excess carrier concentrations, performance of the sample can be identified by analyzing PL intensity observed on the PL imaging photo [54]. Another advantage of the PL imaging technique is that it is contactless; therefore, it can be used for process monitoring before and after processing stages, for instance, during the solar cell fabrication process [54].

The PL intensity numerical analyzation method is a technique for detailed characterization of silicon solar cells by developing PL intensities collected from an incident laser at different wavelengths. In the PL intensity numerical analyzation technique, the solar cell sample is illuminated under a laser source with at least two different wavelengths. PL intensity measurement changes with the wavelength when the sample has been illuminated under the laser source with different wavelengths. The optical properties of the sample can be identified by using numerical methods to analyze 
the PL intensity difference. For b-Si substrates, the b-Si geometry effect on PL intensity and the surface recombination effect on carrier lift time can be studied through numerical methods on the PL intensity deference. Based on results from the PL intensity numerical analysis technique, performance of the b-Si substrate on solar cell applications can be identified directly after the etching process without completing the standard solar cell fabrication process, for example, fabrication of the surface contact layer [55].

Unlike the PL measurement method, which is only used once to measure the experimental results of the b-Si solar cell after fabrication, the SEM images are more frequently used during the fabrication process to ensure the accuracy of b-Si structures. As we discussed in the previous sections, the geometry of b-Si structures can be affected by various parameters during the ECR etching. Moreover, the geometry of b-Si structures could affect the total cell efficiency by increasing or decreasing the surface reflectivity. The geometry of b-Si structures has to be carefully studied and monitored during the fabrication process to match the theoretical design. However, the geometry of b-Si structures and defective structures on b-Si solar cell are at the nanometer scale, which made them difficult to study under ordinary conditions. By using the SEM system with nanoscale resolution, features of b-Si structures and defective structures on solar cells could be easily identified within a short time period. 


\subsubsection{Silicon Nanowires Solar Cell}

Based on our research from Chapter 2, the horizontally-grown SiNWs fabrication process is a three-step growing process. Firstly, deposit uniform gold ( $\mathrm{Au}$ ) thin films on the substrate; secondly, anneal the substrate at high temperature to form an array of Au droplets on the substrate; thirdly, evaporate the substrate at designed temperature, pressure and low Si deposition speed with designed reaction time. The extremely low growth rate, especially when compared to the chemical vapor deposition (CVD) method, is one of the reasons for the observed horizontal growth of silicon nanowires. The direction of Si nanowire growth depends on the orientation of the substrate. For example, if the SiNWs substrate is a c-Si wafer with $<100>$ orientation, Au droplets formed on the front will be propelled forward along two orthogonal $<011>$ directions by the growing silicon nanowires. The result is a formation of an orthogonal network of SiNWs with a topological complexity and connectivity that depends on the growth time as well as the starting diameter of the Au droplets.

The most important feature discussed in the operation of SiNW c-Si solar cell is the ability to reduce surface reflectivity at the front surface. The absorption coefficient of cSi solar cell is between $400 \mathrm{~nm}$ to $1100 \mathrm{~nm}$ wavelength. Because the width and the height of SiNWs can be designed closed to or less than the absorption coefficient of c-Si solar cells, it will be able to reduce surface reflectivity of the substrate at the absorption wavelength range by the diffraction effect. As surface reflectivity decreases, more photons will be able to operate in the substrate and generate more electrons. Thus, a c-Si solar cell with a high surface coverage of SiNWs operates at a higher cell efficiency enhancement than the solar cell with a low surface coverage. As we know from the 
literature review chapter, the length of SiNWs depends on the growth time, the width of SiNWs depends on the diameter of the Au droplet, and the direction of SiNW growth depends on the crystal orientation of the c-Si substrate. Since p-type c-Si solar cells with the $<100>$ orientation are the most commonly used c-Si solar cells in research facility, solar cells with $<100>$ orientation are used as substrates for SiNW growth, and SiNWs are propelled forward along the orthogonal $<011>$ direction. The diameter of the $\mathrm{Au}$ droplet depends on the thickness of Au film deposited on the substrate. Due to the limitation of fabrication equipment, only Au film of $1 \mathrm{~nm}$ thickness can be uniformly deposited on the silicon-based substrate. The average diameter of the Au droplets formed by uniform Au film of $1 \mathrm{~nm}$ thickness is $90 \mathrm{~nm}$. Hence, the growth time is the most important parameter, which will affect the geometry of SiNWs and the surface coverage on the substrate.

In accordance with the VLS growth model, liquid droplets then capture material from the vapor and deposit it onto the solid in the form of nanowires, with longer silicon growth time resulting in longer nanowires [56]. Longer SiNWs means more surface area will be covered by SiNWs, which is of benefit to cell efficiency enhancement. Therefore, c-Si solar cells with longer growth time will have higher cell efficiency enhancement than c-Si solar cells with short growth time. On the other hand, longer growth time will increase the probability of losing wire shape on SiNWs. Increasing growth time will decrease average separation distance between SiNWs, which may cause them to gather together to form a random shape of structure. Final, the optimal parameter of SiNWs on c-Si solar cells is longer growth time of as long as the shape of the nanowires remains 
constant. In this thesis, all of the SiNWs were grown by Grace Ruiping from the University of Western Ontario. 


\section{Experimental Design}

The c-Si solar cells used for the cubic silver (Ag) MNPs deposition were fabricated by the CUMFF at the beginning of 2013 and 2014. The fabrication processes for the solar cells followed the same process used in the ELEC 4703 Solar Cell course in the winter terms of 2013 and 2014. The students from the courses designed the finger grid on the solar cell in small groups. The parameters used for finger grid design were based on the students' ID numbers and were not the optimal parameters for cell efficiency. The cell efficiency from each solar cell fabricated by the student group was different. The only variation in the fabrication processes used in the thesis is the diffusion temperature. There were three diffusion temperatures used for the solar cells fabricated in 2013, which were $850{ }^{\circ} \mathrm{C}, 875^{\circ} \mathrm{C}$, and $1000{ }^{\circ} \mathrm{C}$. The solar cells fabricated in 2014 had two diffusion temperatures at $875^{\circ} \mathrm{C}$ and $1000^{\circ} \mathrm{C}$, respectively. The fabrication runsheet for the solar cells fabricated in the ELEC 47032013 winter term is included in Appendix B.

\subsection{Plasmonic Solar Cell}

The fabrication method of cubic Ag MNPs deposited on the c-Si solar cell was designed by A. Bottomly from the Department of Chemistry at Carleton University. After the simulation processes had been performed by the FDTD simulator, we worked together to design the size and the surface coverage of cubic Ag MNPs in the chemistry Lab. The cubic Ag MNPs were synthesized in the chemistry lab based on the facile synthesis method of cubic Ag MNPs and Au nanocages from the literature [57]. The deposition method used in this project was the Langmuir-Blodgett (LB) monolayer technique. The LB monolayer technique could use to deposit single or multiple nanoscale monolayers onto a solid substrate, first proved by Dr. Irving Langmuir in 1917 [58]. 


\subsubsection{Silver Metal Nanoparticles (MNPs) Fabrication Method}

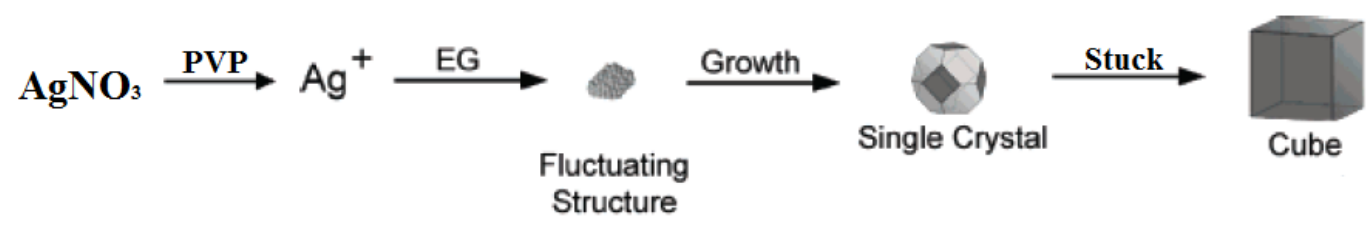

Figure 4-1: Illustration of the reaction path leading to well-defined cubic Ag MNP [42].

The fabrication processes of cubic Ag MNPs follow to the procedure described in Adam's literature [57]. Fig. 4-1 above shows an illustration summary of the cubic Ag MNP growth process beginning with silver nitride $\left(\mathrm{AgNO}_{3}\right)$. Typically, $\mathrm{AgNO}_{3}$ and polyvinylpyrrolidone (PVP) were injected into a flask containing ethylene glycol (EG) set to $150{ }^{\circ} \mathrm{C}$. Ag ions were released from the $\mathrm{AgNO}_{3}$ in $\mathrm{EG}$ solution after the PVP was added to the reaction. EG reduces $\mathrm{Ag}$ ions to $\mathrm{Ag}$ atoms in high temperature conditions, which in turn come together to form Ag nuclei. At nanoscale size and in a high temperature environment, Ag nuclei have enough thermal energy for the structure of the nuclei to fluctuate, allowing Ag nuclei to form depending on their energetic favorability. Fluctuation structure decreases as Ag nuclei grows, until they evolve into single crystal seeds. After Ag single crystal seeds grow past a certain size of Ag seeds, they get stuck in a single crystal to become an cubic Ag MNP [59]. The reaction is quenched by rapidly removing the thermal energy from the solution when the specific wavelength is observed in a UV-visible spectroscopy. For example, in order to synthesize cubic Ag MNPs with a $55 \mathrm{~nm}$ in edge length, the reaction was quenched by removing the flask from the hearting source and placing it in an ice bath when a distinct peak at $350 \mathrm{~nm}$ was observed in the spectrum [60]. 
In order to fabricate cubic Ag MNPs with optimal performance, there are two critical points during the synthesis process: control the size of cubic Ag MNPs and protect cubic shape at corners during the reaction. The key to controlling the size of cubic Ag MNPs is to stop the reaction at specific times immediately. Because the size of cubic Ag MNPs is controlled by the reaction time and the size of cubic Ag MNPs cannot change without thermal energy, quenching the reaction at the designated reaction time immediately without extra processing time provides cubic Ag MNPs with minimal size differences. Since the cubic Ag MNPs are synthesized from Ag ions alone with thermal energy, substantially longer reaction times determine cubic Ag MNP size and the rounding of their corners. As reaction time increases, the size of cubic Ag MNPs increases, and corners of cubic Ag MNPs become less distinct. Due to the synthesis method and the chemical solution used in the method, the maximum size of cubic Ag MNPs with distinct corners is approximately $90 \mathrm{~nm}$ in edge length [57]. Cubic Ag MNPs with a size larger than $90 \mathrm{~nm}$ in edge length appear to have round corners by using this synthesis method. As Ag ions substantially grow on the cubic Ag MNPs, cubic structure becomes spherical after corners disappear. Fig. 4-2 below shows an SEM image of cubic Ag MNPs with 90 $\mathrm{nm}$ in edge length in the solution and a photo of a flask containing cubic Ag MNPs after the reaction. 


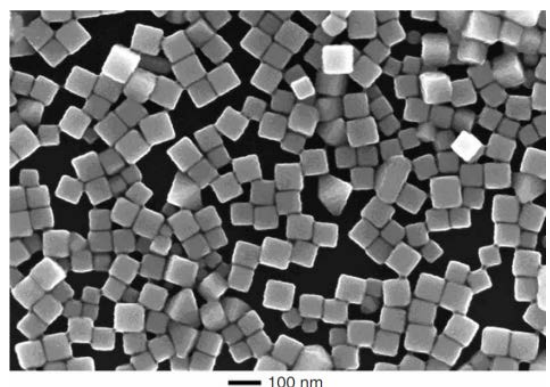

(a)

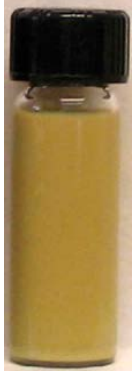

(b)

Figure 4-2: (a) SEM image of cubic Ag MNPs with $90 \mathrm{~nm}$ in edge length stuck together in the reaction solution after the reaction [40] and (b) photo of a flask containing cubic Ag MNPs with 90 $\mathrm{nm}$ in edge length stored in the cubic Ag MNPs solution after the reaction [40]

\subsubsection{Silver Nanoparticles Deposition Method}

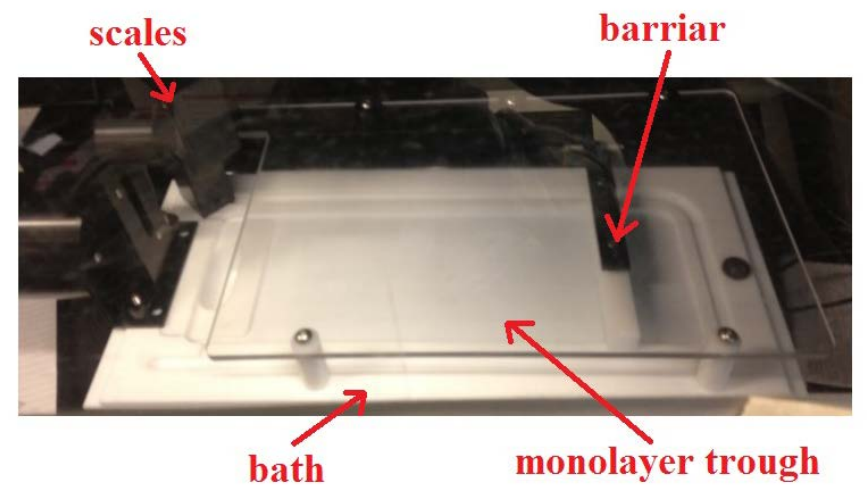

Figure 4-3: Photo of Langmuir-Blodgett (LB) monolayer trough used to prepare cubic Ag MNPs monolayer and deposit the monolayer on solar cells

In order to deposit cubic Ag MNPs on solid substrate, cubic Ag MNP solution from the synthesis reaction has to be purified to remove defective materials left in the solution.

Purification of colloidal cubic Ag MNPs solution collects Ag ions remaining in the solution and washes cubic Ag MNPs synthesized from the reaction. The cubic Ag MNP solution after purification and the washing process is ready to be deposited on the substrate by the Langmuir-Blodgett (LB) monolayer deposition technique. The cubic Ag MNPs deposition process using the LB technique can be separated into two parts:

preparation of the LB monolayer of cubic Ag MNPs and deposition of the LB monolayer on the substrate. Fig. 4-3 above shows a photo of the water trough used to prepare the LB 
monolayer of cubic Ag MNPs in the Department of Chemistry Laboratory. Briefly, the LB monolayers of cubic Ag MNPs samples were prepared by using a water trough filled with standard purified water. An appropriate amount of cubic Ag MNPs solution with colloidal liquid mixtures was slowly deposited onto the water surface to form a cubic $\mathrm{Ag}$ MNP monolayer. Moreover, cubic Ag MNPs were nonuniformly distributed in the monolayer due to the interparticle force from the colloidal liquid mixture. Then, several isotherm cycles were performed to anneal the LB monolayer to design surface pressures in the solid phase before depositing the monolayer on the solar cell. Monolayers of cubic Ag MNPs and colloidal liquid mixtures were transferred onto the solar cell at various surface pressures by slow, vertical dipping at room temperature. At the end, the solar cell with the LB monolayer on the top was washed with aqua regia of low concentration to remove the colloidal mixtures left on the surface [60]. Surface pressure of the monolayer was measure by using the Wilhelmy plate method.

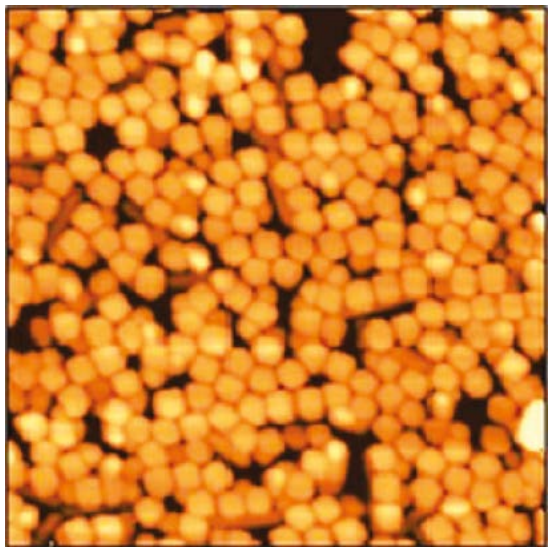

(a)

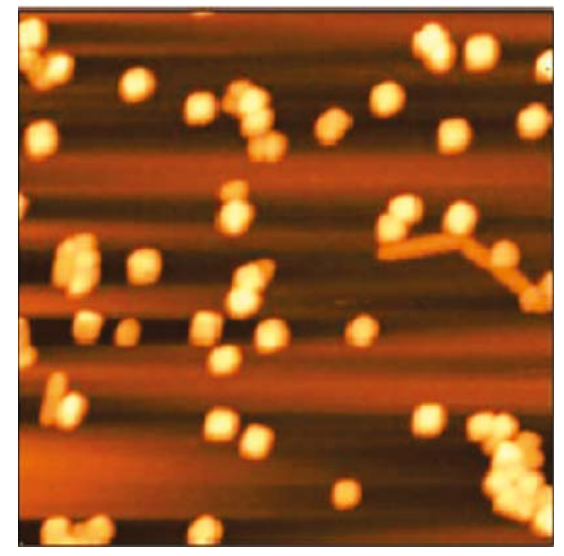

(b)

Figure 4-4: AFM topography images of (a) cubic Ag MNPs in the MNPs solution after the synthesis reaction and (b) substrates of cubic Ag MNPs mixed with phospholipid molecules mixture liquid [41]

Fig. 4-4 above shows atomic force microscopy (AFM) topography images of cubic Ag MNPs on the substrate. Fig. 4-4 (a) is before the LB monolayer preparation, and Fig. 4-4 
(b) is after the LB monolayer preparation. Cubic Ag MNP mixture before the LB monolayer preparation process appeared to have nonuniform distribution and various overlapping in a three-dimensional system, with near-zero interparticle distance and multi cubic Ag MNPs overlapping in small spacing, even at low surface pressures due to their hydrophobic nature. Based on the literature, MNP dimers separated by a distance more than $1 \mathrm{~nm}$ exhibit a strong electromagnetic (EM) field at the junction, whereas an interparticle distance less than $1 \mathrm{~nm}$ reduces the EM field at the junction via electron tunneling [61]. Overlapping MNPs can be treated as a large MNP with random geometry, which affects the deposition of the cubic Ag MNP monolayer on the solar cell due to higher surface pressure than smaller NPs. Control of interparticle distance in the cubic Ag MNPs monolayer has been a challenge in nanoparticle society for many years.

In this thesis, mixtures of phospholipid molecule liquids were used to control interparticle distance and optical properties of cubic Ag MNPs in the monolayer prepared by the LB monolayer technique. By adjusting the surface pressure and the ratio of phospholipid liquid, a more uniform distribution of the cubic Ag MNPs was observed in the monolayer with cubic Ag MNPs and a phospholipid mixture compared to the monolayer with cubic Ag MNPs only. Another advantage of using the phospholipid mixture was its ability to control the interparticle distance even at high surface pressure. Therefore, more cubic Ag MNPs were able to distribute in a small area but maintained their interparticle distance. Fig. 4-5 below shows the AFM images of cubic Ag MNPs deposited on substrates with different surface pressures, at $0.1 \mathrm{mN} / \mathrm{m}, 15 \mathrm{mN} / \mathrm{m}$ and 35 $m N / m$, respectively. 


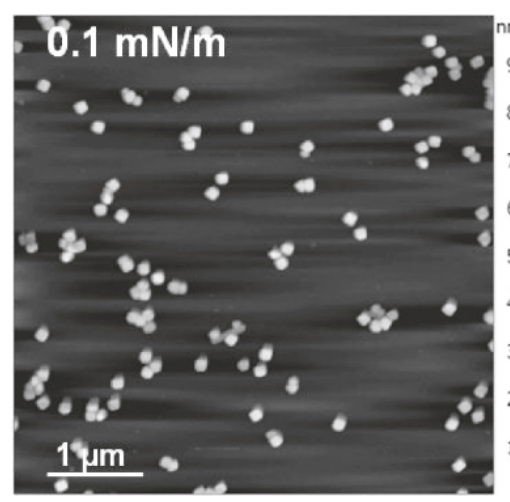

(a)

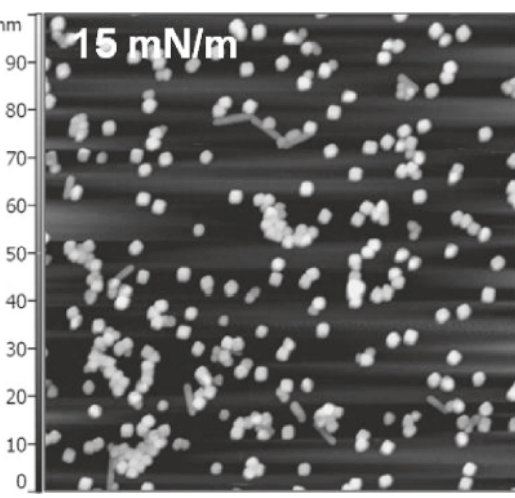

(b)

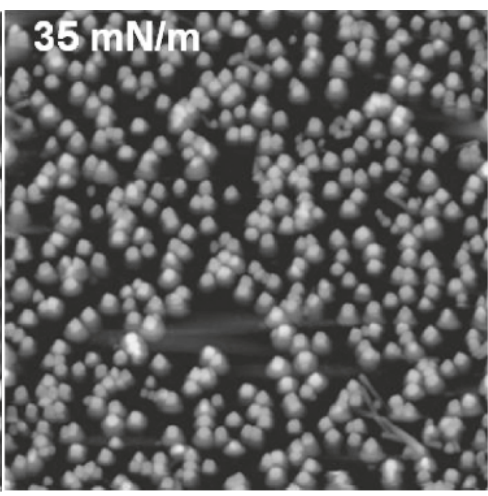

(c)

Figure 4-5: AFM topography images of cubic Ag MNPs mixed with phospholipids mixtures deposited on substrate at different surface pressure, (a) $0.1 \mathrm{mN} / \mathrm{m}$, (b) $15 \mathrm{mN} / \mathrm{m}$, (c) $35 \mathrm{mN} / \mathrm{m}$ [41]

\subsection{Black Silicon Solar Cells}

The black silicon (b-Si) structures were etched by the ECR etching machine in the CUMFF Lab. The gas ratio for the $\mathrm{SF}_{6} / \mathrm{O}_{2}$ mixture was 0.5 , in which the value of input of $\mathrm{SF}_{6}$ gas was 3.65 and the value of input $\mathrm{O}_{2}$ gas was 6.78. The gas ratio used for the experiment was not matched with the gas ratio from the theoretical design. The gas ratio was adapted from previous experiments, which proved to work very well for this ECR machine. We attempted to use other gas ratios during the etching process but were not successful after etching. The gas ratio we had attempted to use for the etching was 1.5 , in which the value of income $\mathrm{SF}_{6}$ was 3.65 and the value of income $\mathrm{O}_{2}$ gas was 2.21. By reducing the input value of $\mathrm{O}_{2}$ gas during the etching, the c-Si substrate under the plasmas ions bombardment had less protection from the $\mathrm{O}_{2}$ gas. The etching speed should be faster in this case.

The temperature of the reaction chamber was controlled around -30 degrees Celsius during the etching process. Nitrogen $\left(\mathrm{N}_{2}\right)$ gas was used as the cooling sources through whole etching process. The choice of this temperature was based on previous experiments. Another reason is that plasma ions had less power at high temperatures, and 
the etching rate was slower. Slowing the etching rate would protect the needle-like structures created by the plasmas etching process from over-etching. The b-Si structures were defined as rough, special-shaped structures remaining on the surface after etching the substrate with plasmas ions. High etching rate would be able to etch the exposed area smoothly with nothing left behind. In the b-Si etching, the roughness of the surface structures remained after the etching process, as required for this thesis. Hence, the ECR etching process took place at $-30{ }^{\circ} \mathrm{C}$ to form the needle-like structures on the c-Si wafer surface.

\subsubsection{B-Si Solar Cell Fabrication Procedures}

The experimental design of the b-Si solar cell in this thesis will start with the experimental design of the fabrication procedure for the b-Si structure from a p-type c-Si wafer. A step-by-step explanation of the fabrication processes for the b-Si solar cell will be discussed in this section after the run-sheet of the fabrication procedure. The $\mathrm{b}-\mathrm{Si}$ structure will be fabricated by using the electron cyclotron resonance (ECR) ion etching machine set up in the CUMFF. 


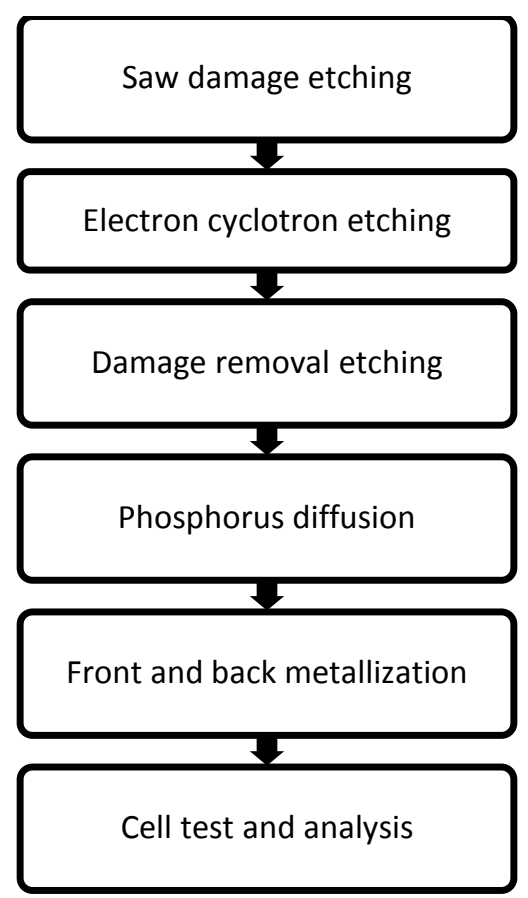

Figure 4-6: Fabrication procedures for c-Si solar cells with ECR texturing

The fabrication procedure is outlined in Fig. 4-6 above. The wafers used in this work are boron-doped p-type c-Si wafers. This $<100>$ p-type c-Si wafer has a 4-inch diameter with a 1-4 $\Omega / \square$ surface sheet resistance. Based on the research from literatures, the type of the c-Si wafer used for the b-Si structures fabrication normally does not affect the etching rate since the etching processes are physical process unrelated to the optical property of the substrate. The difference between a p-type and an n-type c-Si wafer was the diffusion type on the substrate.

The first step of the b-Si solar cell fabrication is to clean the silicon wafer surface. The c-Si wafer will form a thin native dioxide layer on the surface when it is exposed in the air condition for a certain time period. $\mathrm{The} \mathrm{SiO}_{2}$ layer generated on the wafer surface will affect the etching procedure by reducing the etching rate. According to previous experimental results, the etching rate for $\mathrm{SiO}_{2}$ material is 100 times slower than that for 
untreated c-Si in the ECR machine. Moreover, the $\mathrm{SiO}_{2}$ material remaining on the wafer will affect the radio frequency (RF) power used in the etching process. The ECR machine uses the plasmas generated from the reaction gas mixture to etch the c-Si wafer surface. The plasmas used during the etching process contain high energy, and some of them will reflect back to the generator reflected from the substrate. The reflected plasmas will affect the plasmas used for the etching process if the reflected plasmas have too much power. Since the reflectivity of $\mathrm{SiO}_{2}$ and the c-Si wafer is different, the reflected powers from these two materials are different. The reflected power during whole etching process should steady to prevent same the etching speed from being employed all the time. The cleaning process is called an RCA cleaning process. The RCA process has to be done within 5 minutes before the ECR etching to ensure the quality of the wafer surface.

The reaction gas mixtures used in the ECR etching are $\mathrm{SF}_{6}$ and $\mathrm{O}_{2}$. The gas ratio for $\mathrm{SF}_{6}$ and $\mathrm{O}_{2}$ should be close to 1:2 due to the fact that $\mathrm{SF}_{6}$ is used to generate plasmas for etching and $\mathrm{O}_{2}$ is used to prevent the structure from over-etching. If the amount of $\mathrm{SF}_{6}$ gas increases in the etching, the etching speed increases, and the needle-like structure may be unable to exist on the substrate before getting etched. Increasing the amount of $\mathrm{O}_{2}$ gas slows the etching speed and affects the textured structures. The FDTD simulator used for the plasmons investigation is not able to simulate the ECR etching process. Hence, the gas ratio for the $\mathrm{SF}_{6} / \mathrm{O}_{2}$ reaction gas mixture was set to be 0.5 in the theoretical design stage.

A decrease in reflectivity does not automatically lead to an increase in cell efficiency. The ECR etching process not only creates the needle-like structures on the substrate, but it also creates the rough textured structures on the surface of the needle-like structures 
and several defective structures around the uniform structures. ECR generates defects in the near surface region of the c-Si wafer, which reduces the electrical performance of $b$ Si solar cells. The defective structures from the etching process are referred to as surface damage. The surface damage results in a high surface recombination velocity owing to the increased surface area. Due to the surface damage in the c-Si material introduced by energetic ions during the etching process, carriers may have an increased surface recombination, having a great potential of not reaching the p-n junction. Therefore, a high surface recombination velocity reduces quantum efficiency in the short wavelength region, which affects the c-Si solar cell efficiency directly. At the end, a fabrication process called the damage removed etching process is introduced to remove surface damage after the ECR etching process [50]. The DRE process employs a $\mathrm{HNO}_{3} / \mathrm{HF}$ mixture at various etching times to remove the defective structure without increasing the reflectivity. The reaction time and $\mathrm{HNO}_{3} / \mathrm{HF}$ reactive solution ratio are not very clear. The reactive solution ratio will be tested in the experiment in order to determine the optimal parameters for this thesis.

The phosphorus diffusion process to form the $\mathrm{p}$-n junction on the $\mathrm{c}$-Si wafer will begin after the DRE process. The b-Si wafer will dope in a conventional tube furnace using phosphoryl chloride $\left(\mathrm{POCL}_{3}\right)$ as the liquid source. Nitrogen $\left(\mathrm{N}_{2}\right)$ and oxygen $\left(\mathrm{O}_{2}\right)$ gases will be used during the diffusion process. The diffusion temperature used in the thesis will be set to $850{ }^{\circ} \mathrm{C}$. When the diffusion temperature is set to $850^{\circ} \mathrm{C}$, the sheet resistance of the b-Si structure after the diffusion is expected to be approximately $140 \Omega / \square$. Based on this sheet resistance and the approximate initial doping level for the c-Si wafer, junction depth caused by the diffusion process can be calculated. The junction depth for 
the c-Si wafer with an $850{ }^{\circ} \mathrm{C}$ diffusion temperature is estimated to be $500 \mathrm{~nm}$ by the simulator. The height of the needle-like structures is designed to be approximately 1 to 2 $\mathrm{mm}$ and approximately $100 \mathrm{~nm}$ in diameter. This means that the junction depth from the diffusion process should be as long as possible to reach the bottom of the b-Si structure. The junction depth is related to the sheet resistance, where a higher sheet resistance will have a large junction depth. The sheet resistance is controlled by the temperature during the diffusion process. At low diffusion temperatures, the sheet resistance caused by the diffusion process will be larger compared to that from high temperatures. Based on the previous experiments, the $850{ }^{\circ} \mathrm{C}$ diffusion temperature was the lowest temperature used for the fabrication in the thesis. Hence, the diffusion temperature will be set to $850{ }^{\circ} \mathrm{C}$.

The last fabrication process for the b-Si solar cell is to create the surface conductor layer to collect free carriers and conduct a device test. The surface conductor will be fabricated on the front surface of the b-Si solar cell, which is also called the finger grid. The material of the finger grid is silver and approximately $1.2 \mathrm{~mm}$ thick. Since the front surface of the b-Si solar cell is covered by the needle-like structures, those structures located under the finger grid should be removed. $\mathrm{A} \mathrm{SiO}_{2}$ mask covering the surface area without the region under the finger grid is deposited on the b-Si solar cell before the front metallization process. After the $\mathrm{SiO}_{2}$ mask is deposited on the front surface, the ECR etching machine is used to remove the needle structures exposed on the finger grid area. After the ECR etching process in this section, the needle structures under the finger grid are removed from the front surface. The finger grid can be deposited on the b-Si solar cell easily. The ECR etching used in this section is different than that used to create the needle-like structures in the previous section. The gas ratio for $\mathrm{SF}_{6} / \mathrm{O}_{2}$ used in this section 
can be large, e.g., 6 , to remove everything on the exposed area. Since there is very little $\mathrm{O}_{2}$ gas to prevent the structure from over-etching; everything on the exposed area will be etched by plasma ions. The experiment results are required to analyze the etching speed changes due to changing the gas ratio, which is important for obtaining a smooth exposed area for the finger grid.

After the front metallization process, the b-Si solar cells are ready for the experimental measurement. The experimental measurements for solar cell in this project are currentvoltage curve measurement and surface reflectivity measurement. The current-voltage measurement will take place in the Department of Electrical Engineering at Carleton University. The surface reflectivity of b-Si samples will be measured in the SUN Lab at the University of Ottawa.

\subsection{Silicon Nanowire Solar Cell}

The horizontal growth of silicon nanowires (SiNWs) on silicon-based solar cell was fabricated by using the molecular beam epitaxy (MBE) method carried out by Grace Ruiping at the University of Western Ontario. Pieces of single-side-polished p-type predoped single-crystalline silicon (p-Si) wafer with $<100>$ orientation of $10 \times 12 \mathrm{~mm}$ size were used as substrates for silicon nanowire growth. And the substrates have been doped by the phosphorus diffusions process at $875^{\circ} \mathrm{C}$ for 5 minutes using nitrogen $\left(\mathrm{N}_{2}\right)$ and oxygen $\left(\mathrm{O}_{2}\right)$ mixture.

The horizontally-grown SiNWs experiments occurred in an ultrahigh vacuum (UHV) MBE chamber, containing two sources with silicon ( $\mathrm{Si}$ ) and gold $(\mathrm{Au})$ that were evaporated from water-cooled graphite crucibles using electron beam heating. The deposition rate was controlled using the quartz crystal monitors that were calibrated by 
means of independent Rutherford backscattering measurements. The substrates were heated by an electron beam heater and their temperature was controlled by K-type thermocouples. Prior to loading the samples in the UHV MBE chamber, the native silicon oxide layer on the substrate was removed by etching for $60 \mathrm{sec}$ in an HF buffer solution. The substrate was subsequently mounted on a standard sample holder using indium (In) as an adhesive then transferred to the UHV MBE chamber via a load-locking chamber. Any silicon oxide layer that may have been formed during the transfer on the substrates was then removed by outgassing them at $600{ }^{\circ} \mathrm{C}$ for 15 minutes and subsequently heating the substrate at $850^{\circ} \mathrm{C}$ for 10 minutes at $4 \times 10^{-9}$ Torr base pressure prior to the deposition process. After the substrate had cooled to the room temperature, thin Au films of $1 \mathrm{~nm}$ thickness were deposited at $4 \times 10^{15}$ atoms $\cdot \mathrm{cm}^{-2} \mathrm{~min}^{-1}$ growth rate on the substrate. The substrates covered with $1 \mathrm{~nm}$ thick Au film were them annealed at $650{ }^{\circ} \mathrm{C}$ for 30 minutes in order to form Au droplets of $90 \mathrm{~nm}$ diameters. These droplets served as a catalyst for the growth of the ridges, and the reported values correspond to the maximum of $\mathrm{Au}$ droplet size distribution. Silicon that used to form silicon nanowire was deposited at a rate of $5 \times 10^{14}$ atoms $\cdot \mathrm{cm}^{-2} \mathrm{~min}^{-1}$ for 30 minutes to 360 minutes at $650{ }^{\circ} \mathrm{C}$ and $1 \sim 2 \times 10^{-8}$ Torr pressure depending on the theoretical design [62].

After the substrate has been removed from the UHV MBE chamber, a cleaning step involved HF solution will take place on the substrate to remove Au droplets remaining on the front after the nanowire growth process. During the SiNWs growth process, the In adhesive was deposited on the c-Si wafer back surface to connect between the substrate and the chamber holder. After the growth process, the In adhesive was remained on the back surface. It had to be removed in order to process further fabrication procedures. The 
In adhesive was carried out in Aqua regia, a solution composed of $40 \mathrm{~mL} \mathrm{HNO} 3$ added to $160 \mathrm{~mL} \mathrm{HCL}$ and then $200 \mathrm{~mL} \mathrm{H}_{2} \mathrm{O}$ kept at $65^{\circ} \mathrm{C}$. This In material removal process was found to have no negative impact on the Si nanowire or substrate. Next, an aluminum (Al) film with $500 \mathrm{~nm}$ thickness was deposited on the back of substrate by electrical beam evaporation. Finally, a silver/titanium (Ag/Ti) alloy finger grid was applied via electrical beam evaporation system on the front side of substrate. The whole process is detailed pictorially in Appendix C. 


\section{Experimental Results}

\subsection{Plasmonic Solar Cells}

Due to the fabrication and deposition method, the actual cubic Ag MNPs deposited on the c-Si solar cell differed from the solar cell built in the simulator. At first, the fabrication size of cubic Ag MNPs was not always equals to the optimal size designed in the simulations. As we explained in the previous section, the cubic Ag MNPs were synthesized in the lab from the silver mixture solution. By carefully controlling the reaction time, the cubic Ag MNPs were synthesized by collecting many Ag molecules around an Ag seed. The synthesized process of cubic Ag MNPs was reacted fast and with many cubic Ag MNPs at the same time. Depending on distribution of Ag molecules in the reaction solution, the synthesized speed for different cubic Ag MNPs could be different. The difficult part of this fabrication process was to control the synthesized speed for each of them during the reaction. Based on the synthesized system we had in the lab, the fabrication error was estimated to $5 \mathrm{~nm}$ in edge length.

Another difficultly of cubic Ag MNPs fabrication was to control the cubic shape of Ag MNPs. The maximum cubic Ag MNPs size was approximately $100 \mathrm{~nm}$ in edge length. The edge of cubic Ag MNPs was not quite obvious after the size of the cubic Ag MNP becomes greater than $100 \mathrm{~nm}$. Since the cubic Ag MNPs were fabricated by using the synthesized method, the Ag molecules were attracted by the silver seed from different angles. A spherical Ag MNP was the most easily synthesized shape in this condition because the surface attraction on spherical spheres stays the same at any surface location. The cubic Ag MNPs with an edge length greater than 100nm appeared close to the hemispherical shape. 


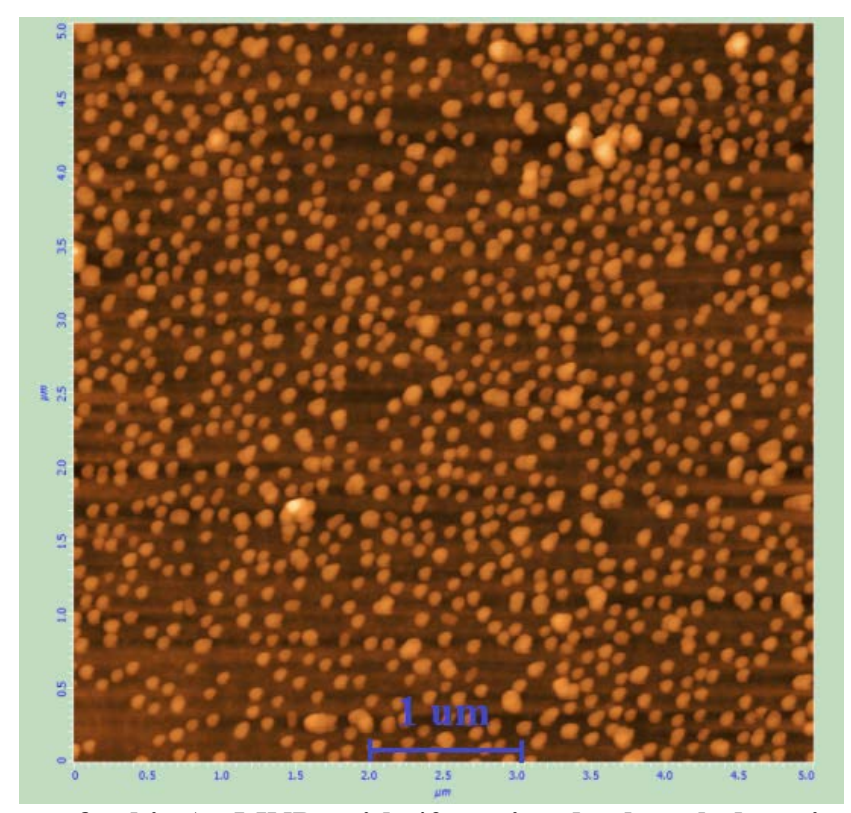

Figure 5-1: AFM image of cubic Ag MNPs with $40 \mathrm{~nm}$ in edge length deposited on a pure c-Si solar cell within $5 \mu \mathrm{m} \times 5 \mu \mathrm{m}$

Fig. 5-1 above shows an atomic force microscope (AFM) image of cubic Ag MNPs with approximately $40 \mathrm{~nm}$ in edge length deposited on a pure c-Si solar cell. The cubic Ag MNPs were nonuniformly distributed on the solar cell surface. At some locations, the cubic Ag MNPs assembled together to form bigger cubic Ag MNPs with a random shape. The bright areas in the image are the assembled cubic Ag MNPs due to their large size. The surface coverage of cubic Ag MNPs from the experiment was difficult to analyze because of the nonuniformly distributed cubic Ag MNPs. Hence, the surface coverage of cubic Ag MNPs could only be roughly estimated in the experiment. 


\subsubsection{Effect of Ag MNPs Size on Efficiency Enhancement}

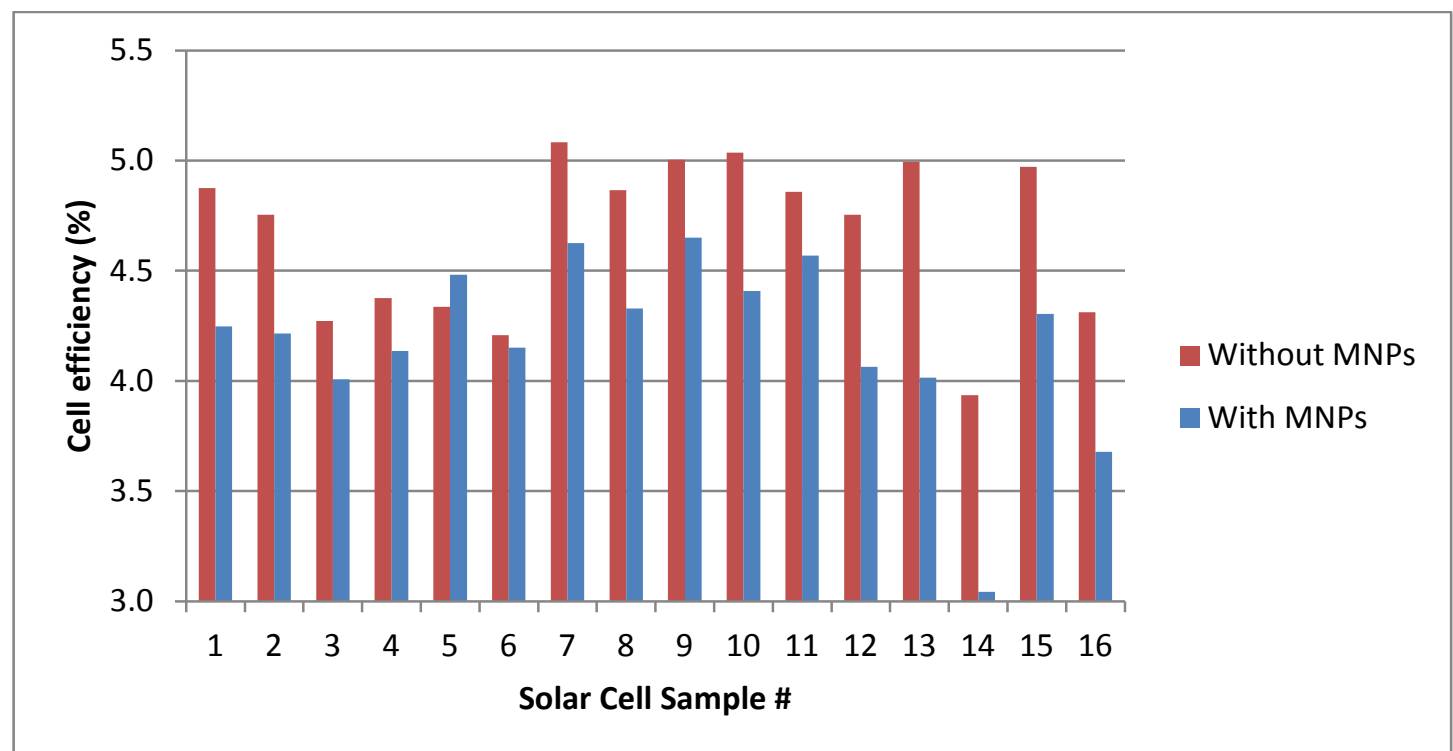

Figure 5-2: Experimental results from c-Si solar cells with and without $40 \mathrm{~nm}$ size cubic Ag MNPs

Based on the simulation results from the previous section, deposited cubic Ag MNPs on the pure c-Si solar cell decreased the solar cell efficiency. The average cell efficiency decreases due to the cubic Ag MNPs deposited on the front by almost $12 \%$ compared to the reference solar cell. Fig. 5-2 shows the experimental results of cubic Ag MNPs deposited on the pure c-Si solar cell. The blue bar in the plot was the cell efficiency of the pure c-Si solar cell before the cubic Ag MNPs deposition, while the red bar on the plot was the cell efficiency of the c-Si solar cell with $40 \mathrm{~nm}$ size cubic Ag MNPs deposited on the top surface. The horizontal axis displays the name of solar cell sample. These experimental results were measured from the c-Si solar cells with the cubic Ag MNPs deposited on the front, as explained in Fig. 5-1. All of these solar cells followed the same Ag MNPs deposition method with the same size of MNPs. The cell efficiency difference on those solar cells was mostly caused by the cell efficiency difference between solar cell substrates. 
The experimental results for cubic Ag MNPs deposited on the c-Si solar cell matched the simulation results under the same conditions. In the simulation results, cubic Ag MNPs with an $80 \mathrm{~nm}$ edge length and deposited on a pure c-Si solar cell decreased the cell efficiency from $1.5 \%$ to $16 \%$ depending on the surface coverage. The size of cubic Ag MNPs used in the experiment was smaller than the size of cubic Ag MNPs used in the simulation because the fabrication method used for cubic Ag MNPs could not be perfectly controlled. Compared to cubic Ag MNPs with large sizes, it is easier to manipulate the shape for cubic Ag MNPs with smaller sizes.

According to the concept of LSPR on MNPs, the absorption ability was primarily due to excitation of small cubic Ag MNPs. The cubic Ag MNPs with small size had less cell efficiency enhancement from their scattering ability than the large MNPs. Hence, the cubic Ag MNPs used in the experiment should have smaller cell efficiency than the cubic Ag MNPs used in the simulation results since the size of cubic Ag MNPs used in the experiment was smaller than the cubic Ag MNPs used in the simulation. Based on the simulation results in Section 3.3.2, the optimal size of cubic Ag MNPs was $80 \mathrm{~nm}$ in edge length. 


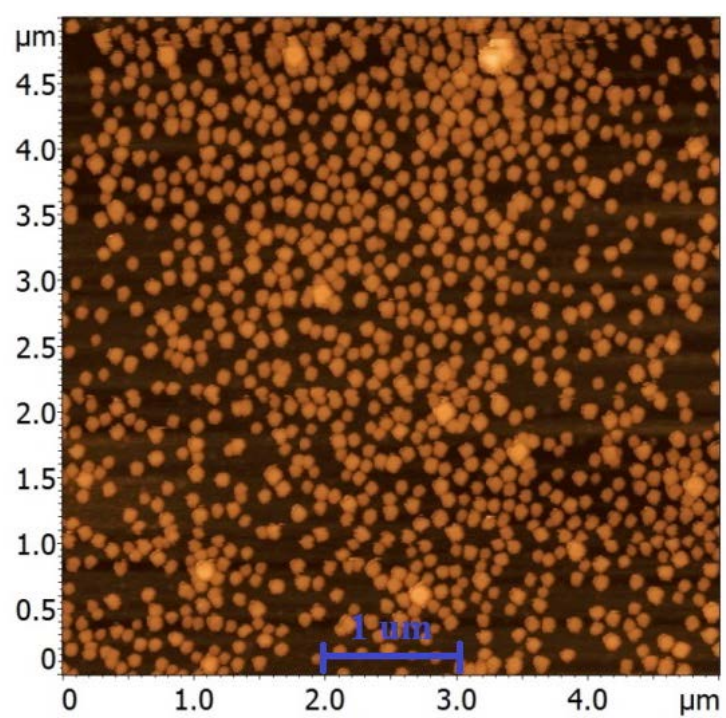

Figure 5-3: AFM image of cubic Ag MNPs with $75 \mathrm{~nm}$ in edge length deposited on the c-Si solar cell

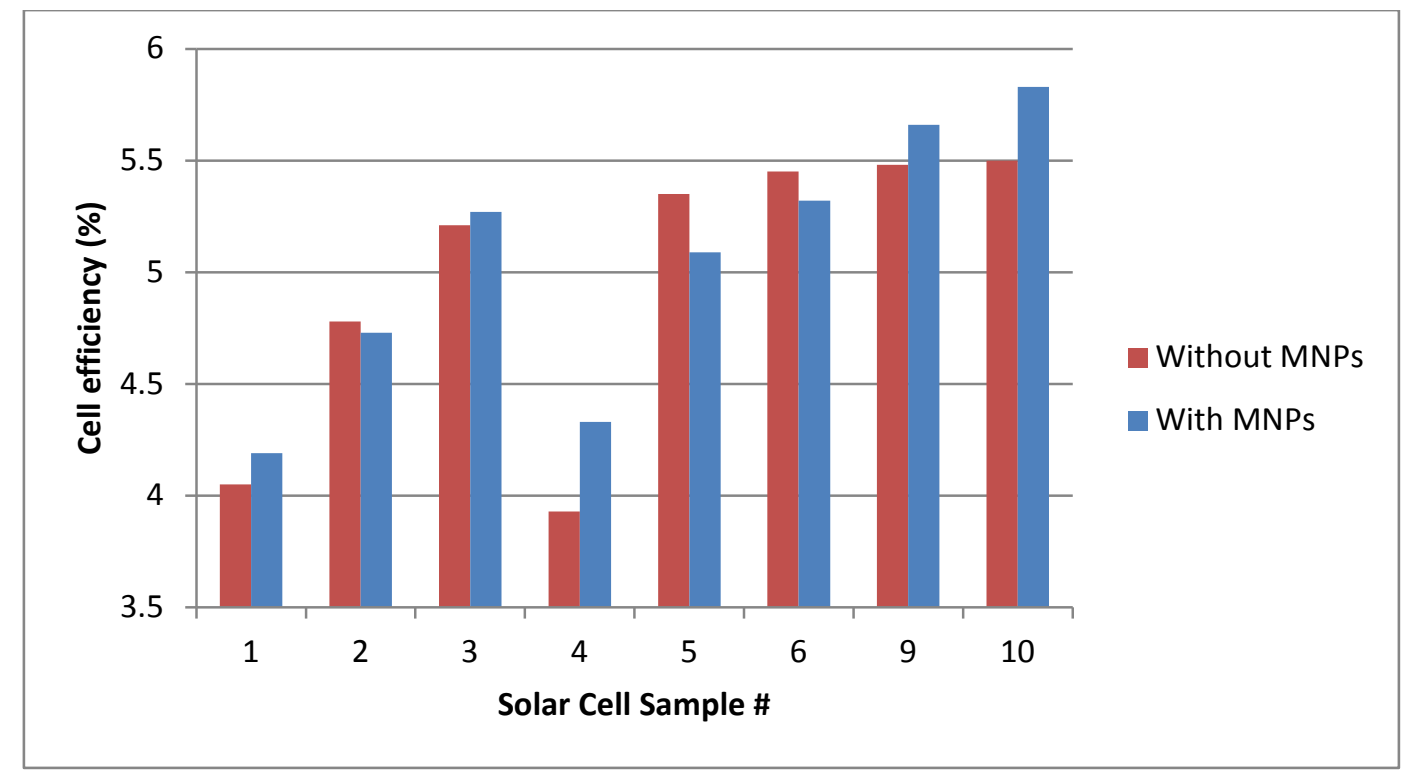

Figure 5-4: Experimental results of the c-Si solar cell with and without $75 \mathrm{~nm}$ size cubic Ag MNPs

Fig. 5-3 above shows the AFM image of cubic Ag MNPs with $75 \mathrm{~nm}$ edge length

deposited on the c-Si solar cell. According to the simulation results in Section 3.3.2, large cubic Ag MNPs have better scattered incident light. From the simulation results, the c-Si solar cell with cubic Ag MNPs by $40 \mathrm{~nm}$ in edge length and 10\% surface coverage has $3.85 \%$ efficiency, while the solar cell with large cubic Ag MNPs, $80 \mathrm{~nm}$ in edge length, has 3.99\% efficiency. The larger cubic Ag MNPs could reduce the diminished cell 
efficiency due to the shadow effects. The figure above shows the AFM image of cubic Ag MNPs deposited on the pure c-Si solar cell front surface. The size of cubic Ag MNPs was approximately $75 \mathrm{~nm}$ in edge length. Fig. 5-4 shows the experimental results of the c-Si solar cell with cubic Ag MNPs deposited on the front surface.

Compared with the experimental results from the solar cell with $45 \mathrm{~nm}$ size cubic Ag MNPs, the $75 \mathrm{~nm}$ size cubic Ag MNPs deposited on the c-Si solar cells showed less efficiency than the cells without MNPs. The experimental results matched the MNP concept from the previous section, in which the large MNPs seem to have higher scattering ability. According to the experimental results for the solar cells with $75 \mathrm{~nm}$ size cubic Ag MNPs, cubic Ag MNPs deposited on five samples (Solar Cell \#1, \#3, \#4, \#9 and \#10 in Fig. 5-4) showed increases in cell efficiency compared to the original solar cell. The average cell efficiency enhancement from these five samples was $4.8 \%$.

As the size of cubic Ag MNPs increased, the scattering ability increased, which increased the cell efficiency enhancement from the cubic Ag MNPs. Increasing the size of MNPs increases the red-shifting of resonance wavelength. When the cell efficiency enhanced by the scattering ability was more than the cell efficiency decrease by the shading losses, the solar cell after cubic Ag MNPs deposition had higher cell efficiency than the solar cell before deposition. Another reason for the cell efficiency increase is related to the cubic Ag MNPs surface coverage. Since the cubic Ag MNPs were nonuniformly distributed on the front of the solar cell, the surface coverage of cubic Ag MNPs was different from one cell to the other. The solar cell with less surface coverage should have less of a cell efficiency decrease caused by the shading losses. The solar cells 
with increased cell efficiency after the cubic Ag MNPs deposition in general had smaller surface coverage.

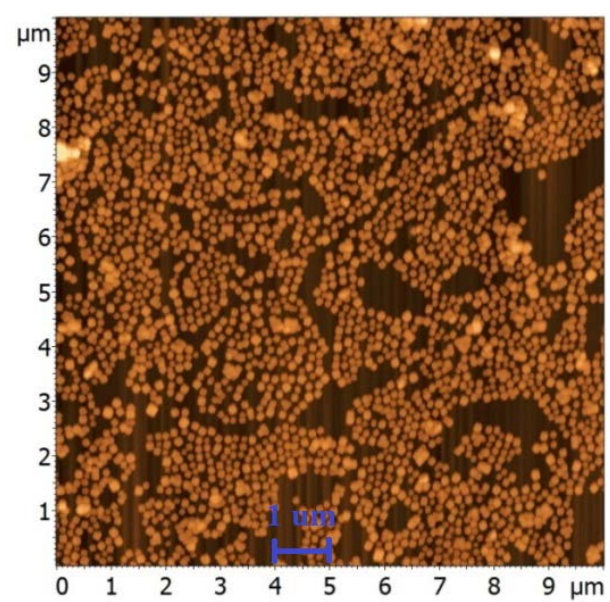

Figure 5-5: AFM image of $80 \mathrm{~nm}$ size cubic Ag MNPs deposited on the c-Si solar cell

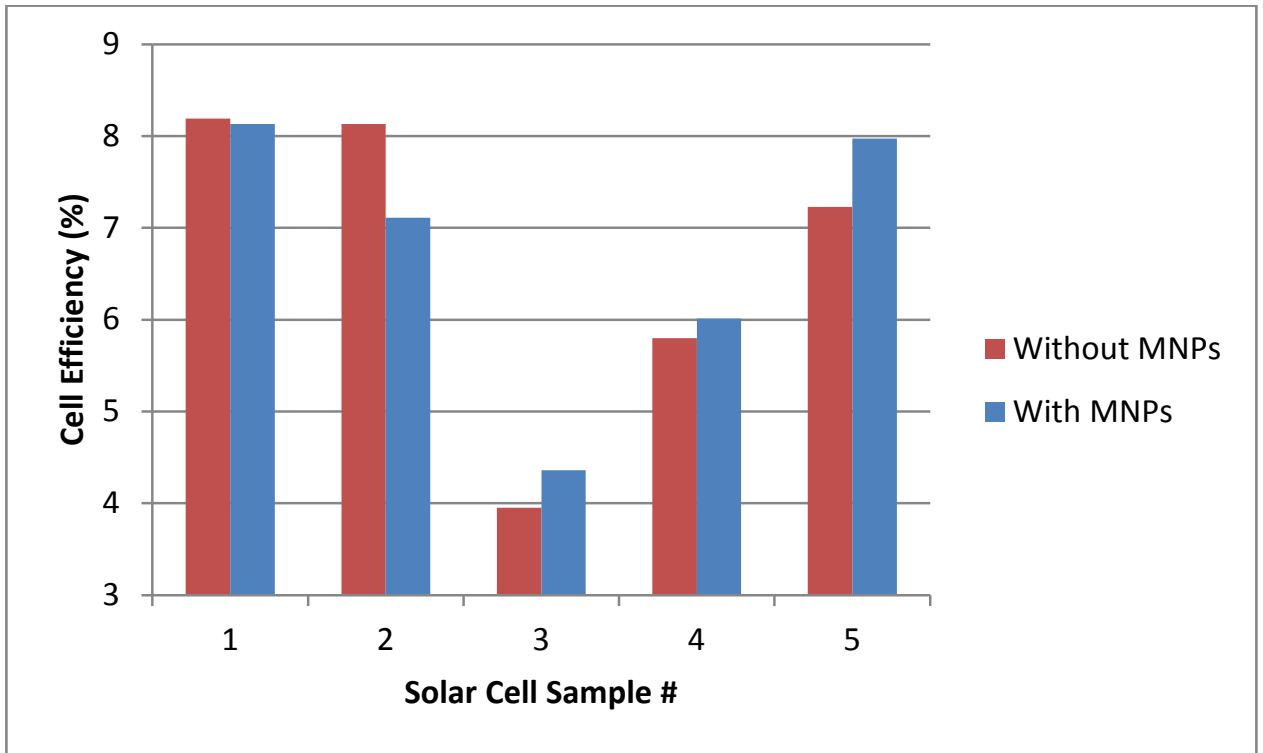

Figure 5-6: Experimental results for the solar cells with $80 \mathrm{~nm}$ size cubic Ag MNPs

Increasing the size of cubic Ag MNPs would increase their scattering ability. The maximum cubic Ag MNPs could fabricate by the synthesized method used in this thesis was around $80 \mathrm{~nm}$ in edge length. Fig. 5-5 above shows the AFM image of $80 \mathrm{~nm}$ size cubic Ag MNPs deposited on the c-Si solar cell, and Fig. 5-6 above shows the experimental results for five solar cells with $80 \mathrm{~nm}$ size cubic Ag MNPs on the front 
surface. Three out of five cells showed increased cell efficiency after cubic Ag MNPs were deposited. The average efficiency enhancement from these three samples was 8.09\%. Increasing the size of cubic Ag MNPs changed the cell efficiency by increasing the scattering ability of Ag cube NPs. The average cell efficiency enhancement for five cSi solar cells with $80 \mathrm{~nm}$ size cubic Ag MNPs was 2\%, which was a small enhancement compared to the other techniques used to enhance cell efficiency.

\subsubsection{Effect of Sublayer Material on Efficiency Enhancement}

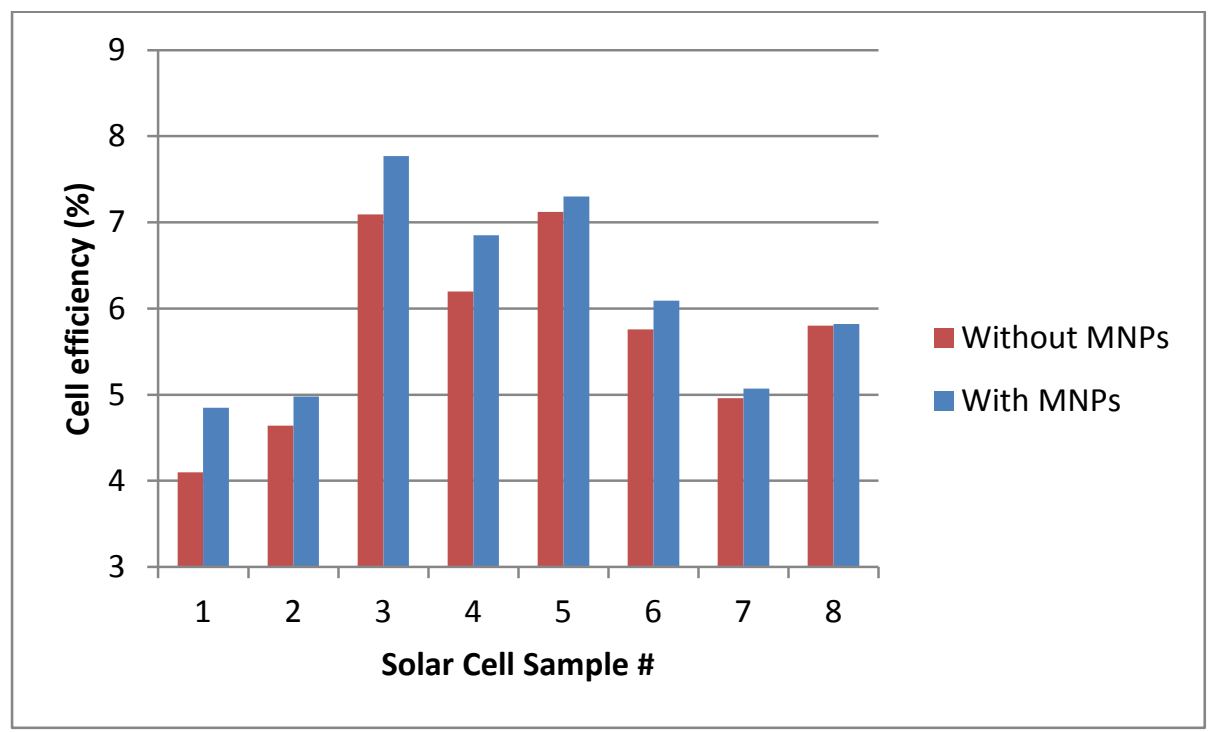

Figure 5-7: Experimental results for the c-Si solar cells with $80 \mathrm{~nm}$ size cubic Ag MNPs deposited on a $25 \mathrm{~nm}$ thickness $\mathrm{SiO}_{2}$ sublayer

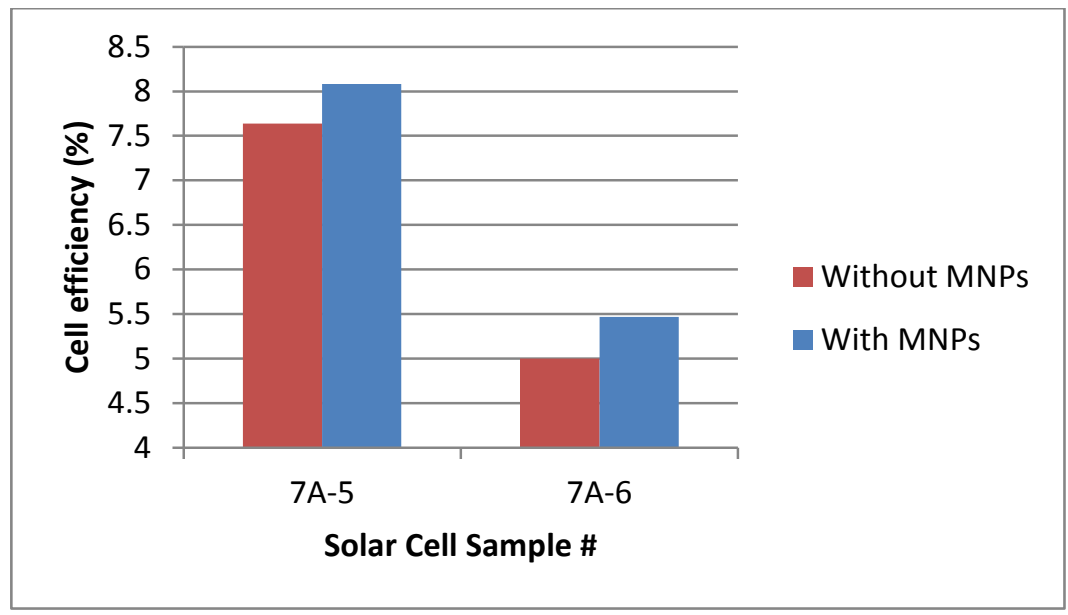

Figure 5-8: Experimental results for the c-Si solar cells with $80 \mathrm{~nm}$ size cubic Ag MNPs deposited on a $35 \mathrm{~nm}$ thickness $\mathrm{SiO}_{2}$ sublayer 
From the simulation design in Chapter 3, we know that the thickness of the sublayer between the solar cell and the MNPs could influence the solar cell efficiency by changing the refractive index of MNPs in the surrounding medium. Fig. 5-7 above shows the experimental results for c-Si solar cells with $80 \mathrm{~nm}$ size cubic Ag MNPs deposited on a $25 \mathrm{~nm}$ thick $\mathrm{SiO}_{2}$ sublayer. Fig. 5-8 above shows the experimental results for c-Si solar cells with $80 \mathrm{~nm}$ size cubic Ag MNPs deposited on the $35 \mathrm{~nm}$ thick $\mathrm{SiO}_{2}$ sublayer. The average size of the cubic Ag MNPs deposited on the sublayer for all samples in Fig. 5-7 and Fig. 5-8 had $80 \mathrm{~nm}$ in edge length.

Fig. 5-7 and Fig. 5-8 show that the thickness of $\mathrm{SiO}_{2}$ sublayer did not drastically change the cell efficiency. With the same size cubic Ag MNPs on the top, the solar cell with a $25 \mathrm{~nm}$ thick $\mathrm{SiO}_{2}$ sublayer had an average of $7.0 \%$ cell efficiency enhancement from the cubic Ag MNPs, while the average cell efficiency enhancement for the solar cell with a $35 \mathrm{~nm}$ thick $\mathrm{SiO}_{2}$ sublayer was $7.5 \%$. Both $\mathrm{SiO}_{2}$ sublayer thicknesses showed efficiency enhancement on the c-Si solar cell samples.

The highest efficiency enhancement was $18.3 \%$ on Solar Cell 1 , with a cell efficiency equal to $4.85 \%$ after the $80 \mathrm{~nm}$ size cubic $\mathrm{Ag} \mathrm{MNPs}$ deposited on a $25 \mathrm{~nm}$ thick $\mathrm{SiO}_{2}$ sublayer on the c-Si solar cell. 


\subsubsection{Effect of Ag MNPs on Surface Reflectivity}

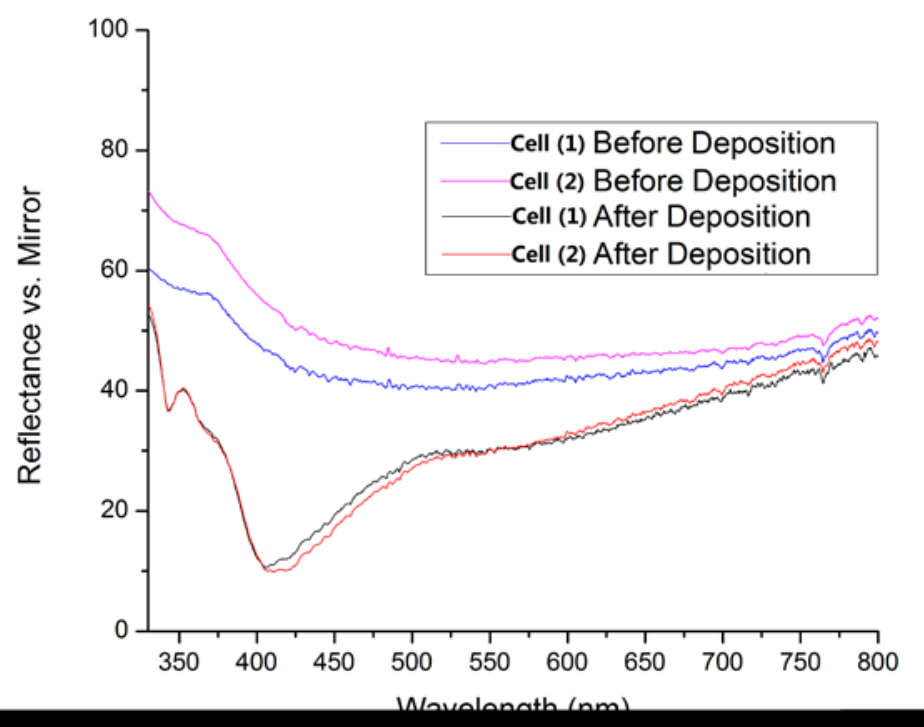

Figure 5-9: Reflection spectroscopy of the c-Si solar cell with 75nm size cubic Ag MNPs deposited on the front surface

Fig. 5-9 shows the reflectance measurement for the c-Si solar cell before and after cubic Ag MNPs deposition. The average size of cubic Ag MNPs deposited on the c-Si solar cell was $75 \mathrm{~nm}$ in edge length. The blue and pink curves are the external reflectance of two c-Si solar cells before MNP deposition. The black and red curves are the external reflectance measured after the cubic Ag MNPs deposition. The external reflectance measurements took place in the Department of Chemistry Laboratory and were performed by Adam Bottomly. The in-house optical reflectance measurement system was built to measure the reflectance of MNPs and semiconductor materials.

According to the experimental results, the reflectance of solar cell was decreased after cubic Ag MNPs on the front surface were deposited. The external reflectance had the lowest reflectance in the $400 \mathrm{~nm}$ wavelength range. After the dip, the reflectance increased with the wavelength. The reflectance of the solar cell with cubic Ag MNPs was 
same as the solar cell without cubic Ag MNPs at around $800 \mathrm{~nm}$. The average reflectance of c-Si solar cells without cubic Ag MNPs was approximately 45\%, but the average reflectance of solar cells with cubic Ag MNPs was approximately 30\%. The average total reflectance reduced from 45 to $30 \%$ due to the cubic Ag MNPs on the front surface. Hence, deposited cubic Ag MNPs on the front surface are experimentally demonstrated to reduce the reflectance on the front surface, which would enhance the amount of incident photons.

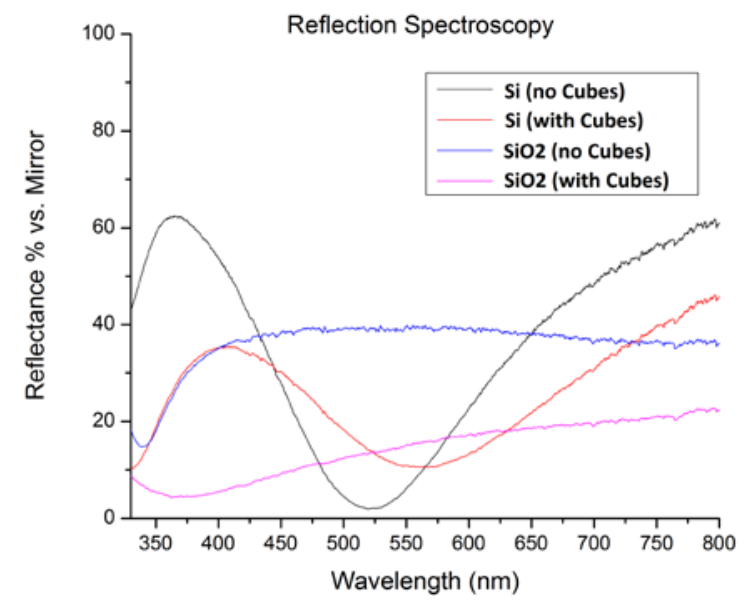

Figure 5-10: Reflection spectroscopy of the c-Si wafer and the $\mathrm{SiO}_{2}$ sublayer with and without $80 \mathrm{~nm}$ size cubic Ag MNPs

Fig. 5-10 above shows the reflection measurements of the c-Si wafer and the $\mathrm{SiO}_{2}$ sublayer with and without cubic Ag MNPs. The size of cubic Ag MNPs averaged $80 \mathrm{~nm}$ in edge length, and the cubic Ag MNPs were deposited on the front surface for both cases. The black and red curves in the plot are the reflectance of the c-Si wafer with and without cubic Ag MNPs. The blue and pink curves were the reflectance of the $\mathrm{SiO}_{2}$ sublayer with and without cubic Ag MNPs.

Based on the reflection spectroscopy, the $\mathrm{SiO}_{2}$ sublayer induced less reflectance than the c-Si wafer. Furthermore, cubic Ag MNPs deposited on both the c-Si wafer and $\mathrm{SiO}_{2}$ sublayer reduced the reflectance. The average reflectance of $\mathrm{SiO}_{2}$ sublayer with cubic $\mathrm{Ag}$ 
MNPs on the front surface was approximately $15 \%$, which was less than the average reflectance of the c-Si wafer with cubic Ag MNPs. Therefore, insertion of a $\mathrm{SiO}_{2}$ sublayer between the c-Si solar cell and the MNPs not only can enhance scattering light from cubic Ag MNPs by changing surrounding media, but also can reduce the surface reflectance to further enhance solar cell efficiency.

\subsubsection{Silver Oxide Effect on Efficiency Enhancement}

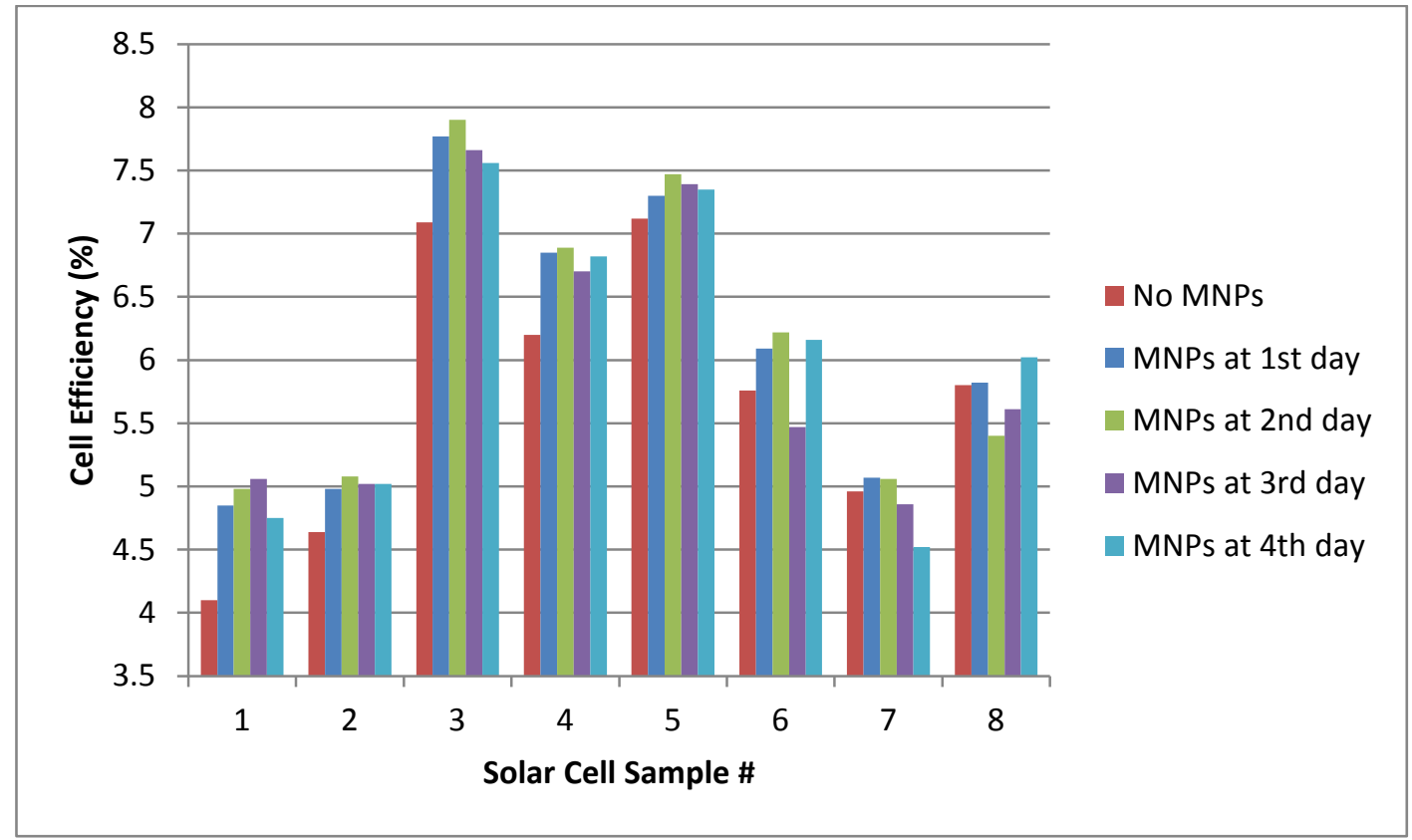

Figure 5-11: Experimental results measured at different days for c-Si solar cells with $80 \mathrm{~nm}$ size cubic $\mathrm{Ag} \mathrm{MNPs}$ and $\mathrm{SiO}_{2}$ sublayer

It is well-known that silver oxidizes when silver material comes in contact with oxygen in the surrounding environment. The oxidation occurred on the cubic Ag MNPs surfaces shortly after deposition on the c-Si solar cell. Fig. 5-11 confirms that the cell efficiency changed with time. The average size of cubic Ag MNPs deposited on the $25 \mathrm{~nm}$ thick $\mathrm{SiO}_{2}$ sublayer was $80 \mathrm{~nm}$ in edge length. This plot provides the cell efficiency 
measurements measured on the first, second, third, and forth days after the cubic Ag MNPs deposition.

Based on the experimental results, the cell efficiencies increased the on second day after the deposition compared to the first day after the deposition for mast solar cells. The reason for this phenomenon was due to the increased size of cubic Ag MNPs from the silver oxide material. As the silver oxide material collected on cubic Ag MNPs surface, the cubic Ag MNPs size increased, and the scattering of light from the MNP also increased. Hence, the cell efficiencies on the second day were higher than those on the first day. On the third day, the cell efficiencies decreased compared to the cell efficiency on the first day. By then, too much silver oxide materials accumulated on cubic Ag MNPs surfaces start to reduce the amount of scattering photons. On the fourth day, the cell efficiencies finally stabilized. After exposure to air on the third day, the cubic Ag MNPs were covered with the silver oxidization on the surfaces, where no more pure silver material was in contact with air/oxygen. The silver oxidation eventually stopped. Hence, the cell efficiencies first decreased after being exposed to oxygen, but eventually the cell efficiency stabilized after the cubic Ag MNPs were covered by silver oxidization. We conclude that silver oxidation proved to lower the cell efficiency by less than $10 \%$ on the c-Si solar cell. 


\subsection{Surface Textured Nanostructures}

\subsubsection{Black Silicon Solar Cells}

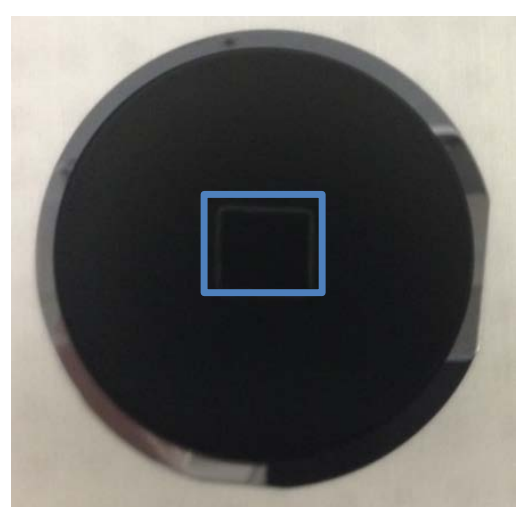

Figure 5-12: b-Si structures sample on the center of vacuum wafer

Fig. 5-12 shows a photo of a b-Si structure sample stocked at the center of a vacuum wafer after ECR etching. The c-Si solar cells with the b-Si structures on top were fabricated in the CUMFF. The ECR etching method was used for the b-Si structures. At the beginning, the b-Si structures were etched on a small piece of c-Si wafer stock. Due to the requirement of the ECR system, the substrate in the system was limited to a 4-inch wafer to ensure proper pressure during the etching process. The etching procedure followed the same procedure described in Chapter 4. The SEM images were used to verify and analyze the needle-like structures formed on the b-Si substrate. The SEM images of b-Si samples were taken from the Nano Imaging Facility at Carleton University. 


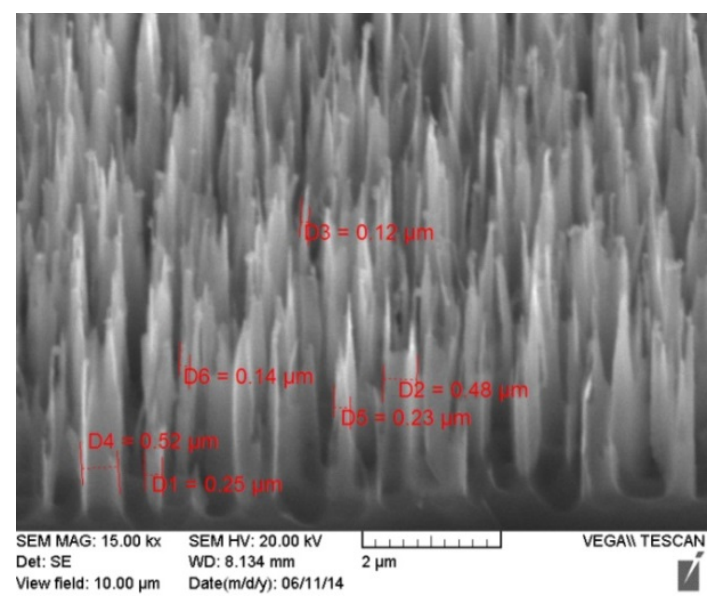

(a)

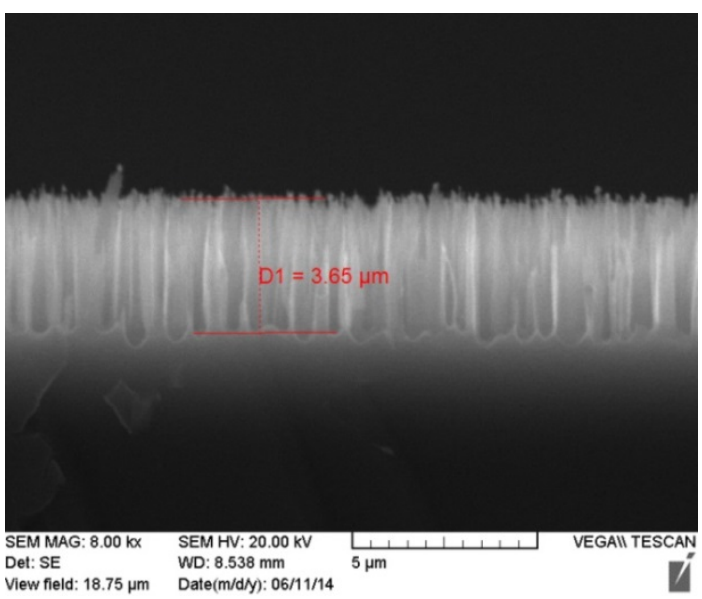

(b)

Figure 5-13: SEM images of b-Si structures etched after 50 minutes from (a) $45^{\circ}$ top view, (b) crosssection view

Fig. 5-13 shows the SEM image of the b-Si structures etched on the c-Si testing sample with an etching time of 50 minutes, where Fig. $5-13$ (a) is the $45^{\circ}$ top view and Fig. 5-13 (b) is the cross-section view. The $45^{\circ}$ top view SEM image was used to verify the needle-like structures and to estimate the width of those structures. The cross-section view was used to verify the height of the needle-like structures.

Based on the needle-like structures displayed in the SEM images, the needle-like structures formed by the ECR etching did not have a uniform width. The average diameter of the b-Si structures was approximately $290 \mathrm{~nm}$ and was calculated. The b-Si structures formed by the ECR etching method had very rough surfaces, and broken structures were visible. Those defective structures on the surface increased the surface recombination rate, which reduced the carriers transported from the incident photons. The cross-section view shows the average height of the needle-like structures was $3.65 \mu \mathrm{m}$ after an etching time of $50 \mathrm{~min}$. There were several non-uniform structures on the bottom of the b-Si samples with heights smaller than the needle-like structures. 


\subsubsection{Effect of Etching time on Geometry}

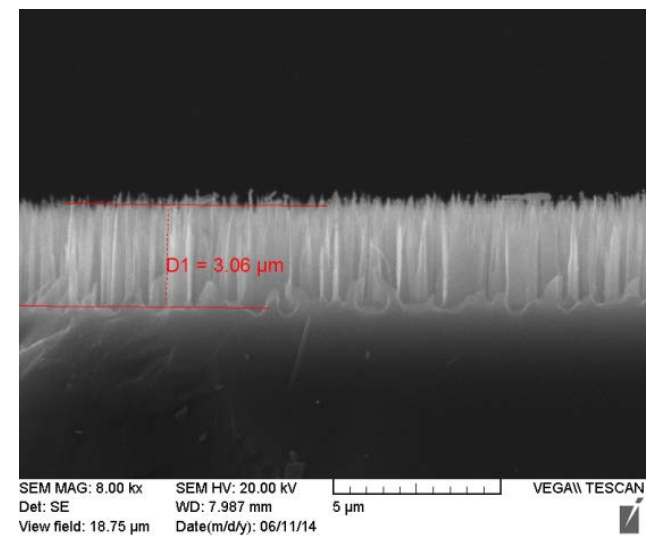

(a)

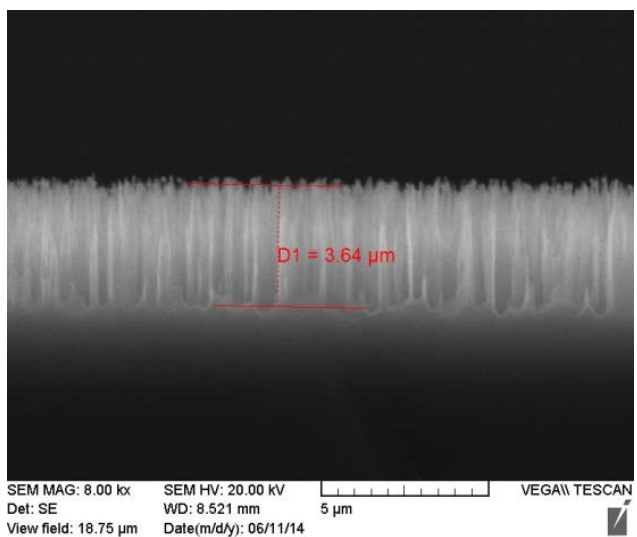

(b)

Figure 5-14: SEM cross-section views of b-Si structures etched after (a) 45 minutes and (b) 50 min

In the ECR etching processes, the etching time was a very important parameter for the needle-shaped structures. The height of b-Si structures formed by the ECR etching process was affected by the etching time. Increasing the etching time increased the height of the needle-shaped structures. Fig. 5-14 shows SEM images of b-Si structures etched at different lengths of time: (a) is after 45 minutes and (b) is after $50 \mathrm{~min}$. The cross-section view of the b-Si sample shows that the average height of the needle-shaped structures was $3.06 \mu \mathrm{m}$ after 45 minutes, while the average height after 50 minutes of etching was $3.64 \mu \mathrm{m}$. Compared with end results, the height of the structures etched was $0.5 \mu \mathrm{m}$ taller when the etch time was longer. Hence, the etching rate of b-Si structures was $0.1 \mu \mathrm{m} / \mathrm{min}$ in the ECR etching system. 


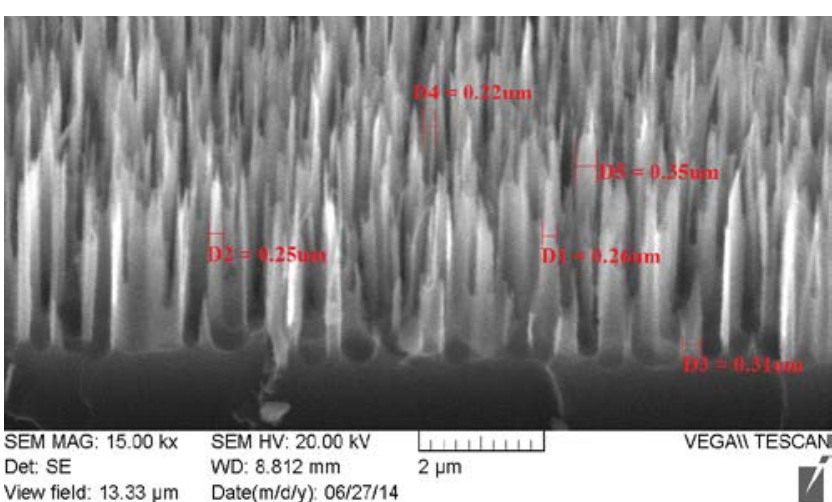

(a)

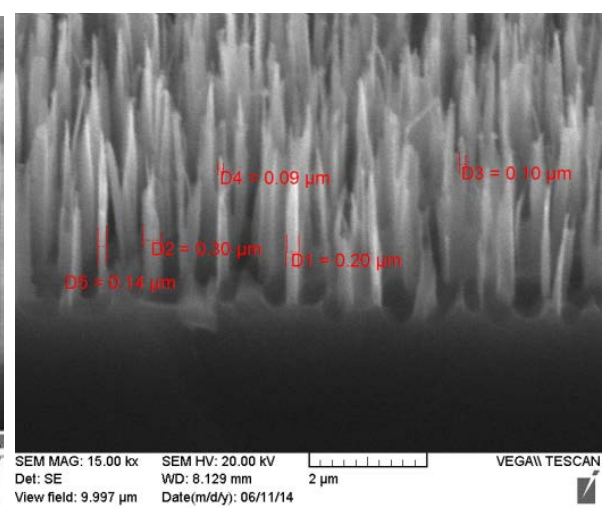

(b)

Figure 5-15: SEM images of needle-like shaped structures width characterization for b-Si solar cells with (a) 45 minutes etching time and (b) 50 minutes etching time

Fig. 5-15 shows the $45^{\circ}$ top view SEM image of the needle-like shaped structures on b-Si solar cells with different length etching times, where (a) is etched after 45 minutes and (b) is after $50 \mathrm{~min}$. Based on the SEM images, the width of each needle-shaped structure was different. The average width of the needle-shaped structures etched after 45 minutes was $0.28 \mu \mathrm{m}$ and $0.17 \mu \mathrm{m}$ when it was etched after $50 \mathrm{~min}$. The b-Si solar cell with a 45 minute etching time had wider needle-like structures than that with a longer 50 minute etching time. In addition, more defective structures were observed after a longer etching time.

The aspect ratio for the b-Si solar cell etched after 45 minutes was 11, while the aspect ratio etched after 50 minutes was 21 . Thus, the b-Si solar cell with a longer etching time had a larger aspect ratio. Now we confirm that the etching time not only affected the height of the needle-like structures, but also their width. As the etching time increased, cSi on the needle-shaped structures surface was continuously removed by the plasma ions so the structures became thinner and taller. Therefore, the aspect ratio of the b-Si solar cell increased relative to height and width. The disadvantage of the b-Si solar cell with a high aspect ratio is the needle-like structures can be easily damaged during the 
fabrication process. Consequently, the b-Si solar cell etched after 50 minutes has more defective structures than the b-Si solar cell etched after the shorter 45 min.

\subsubsection{Effect of Etching Time on Reflectivity}

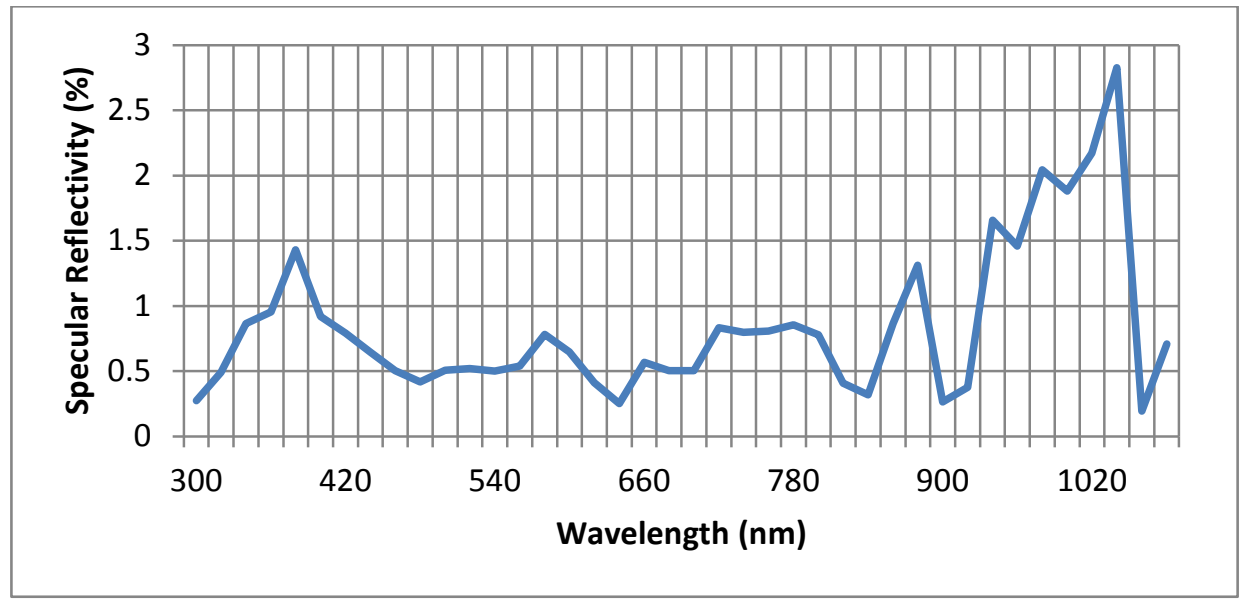

Figure 5-16: Specular reflectivity of b-Si wafer sample etched after 50 min

Fig. 5-16 shows the specular reflectance of the b-Si wafer sample etched after $50 \mathrm{~min}$. The specular reflectance and the reflect power of b-Si structures were measured by Ross Cheriton in the SUN Lab at the University of Ottawa. The specular reflectance measured the entire light spectrum of the c-Si wafer. According to the specular reflectance curve, the average specular reflectance of the b-Si sample was $0.83 \%$. The needle-like structures on the front surface significantly reduced the reflectance from the sample, which matched the simulation design presented in Chapter 4. In order to verify the accuracy of the reflectance measurement, the light intensity difference between the incident power and recovered power was also measured for the b-Si sample. Fig. 5-17 gives the light intensity difference between incident and received light. 


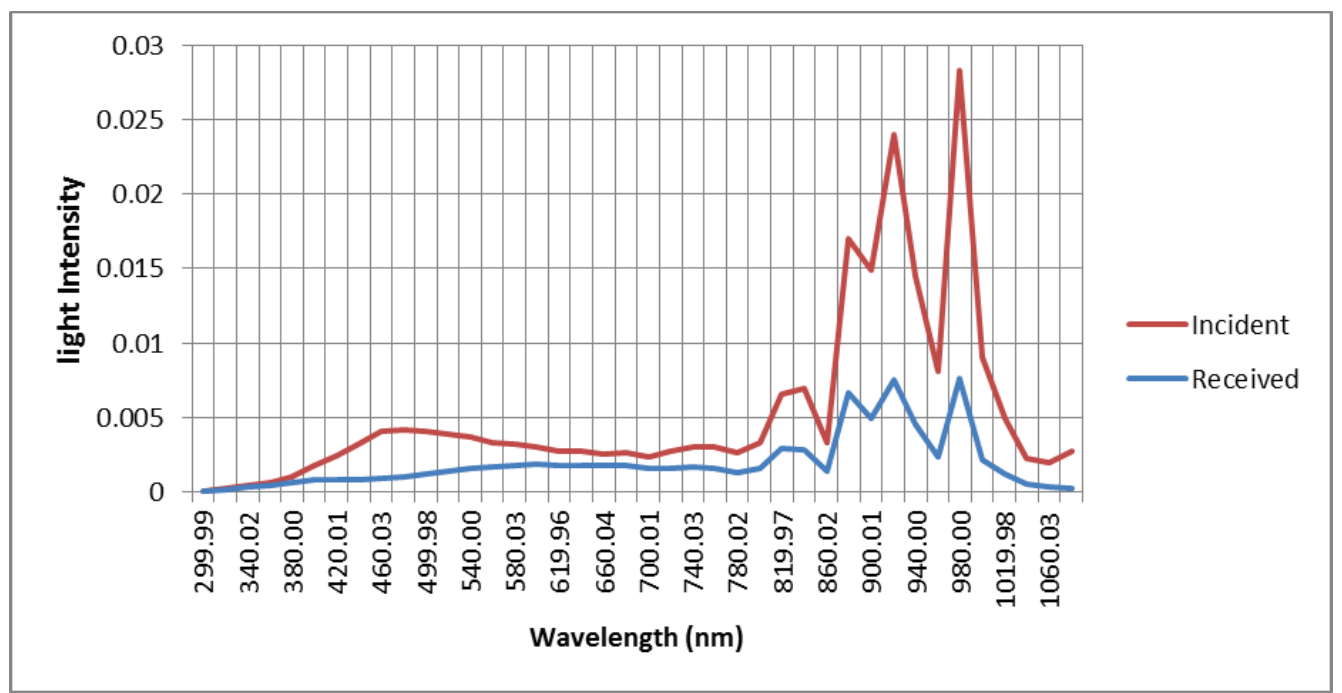

Figure 5-17: Light intensity of the incident light on the b-Si sample and the reflected light from a b-Si sample with 50 minutes in etching time

According to Fig. 5-17, the reflected light from the b-Si sample had the same pattern as the incident light on the b-Si sample. The reflected light had less light intensity than the incident light, which was mainly due to the surface reflectivity. The needle-shaped structures have very small top surfaces with high aspect ratios and demonstrated a very small specular reflectance. Diffused reflectivity was the majority reflectivity that exists on the b-Si structures with high aspect ratios. Since most incident light diffused around the b-Si structure, a second absorption existed on the structures when light was diffused from one to another. These phenomena could increase the solar cell efficiency by increasing the effective path length traveling through the substrate.

The specular reflectance is also related to the flat front surface between the needle-like structures; where the reflected light is at the same angle at which incoming light strikes the surface. However, based on the SEM image of the b-Si solar cell surface at $45^{\circ}$, the needle-like structures are close to each other on the front surface. The flat surfaces between the needle- shaped structures are very small, so the specular reflectance caused by the flat surface should be small. The total surface reflectivity of the b-Si solar cell is 
dominated by the diffused reflectivity. Since the etching time of the b-Si solar cell is only related to the height of needle-shaped structure, changing the etching time of the b-Si solar cell does not affect its specular reflectivity. The comparison of specular reflectivity for the b-Si solar cells with different etching time is not necessary. Hence, we only measured the specular reflectivity of the b-Si solar cell etched after 50 minutes in our experiments.

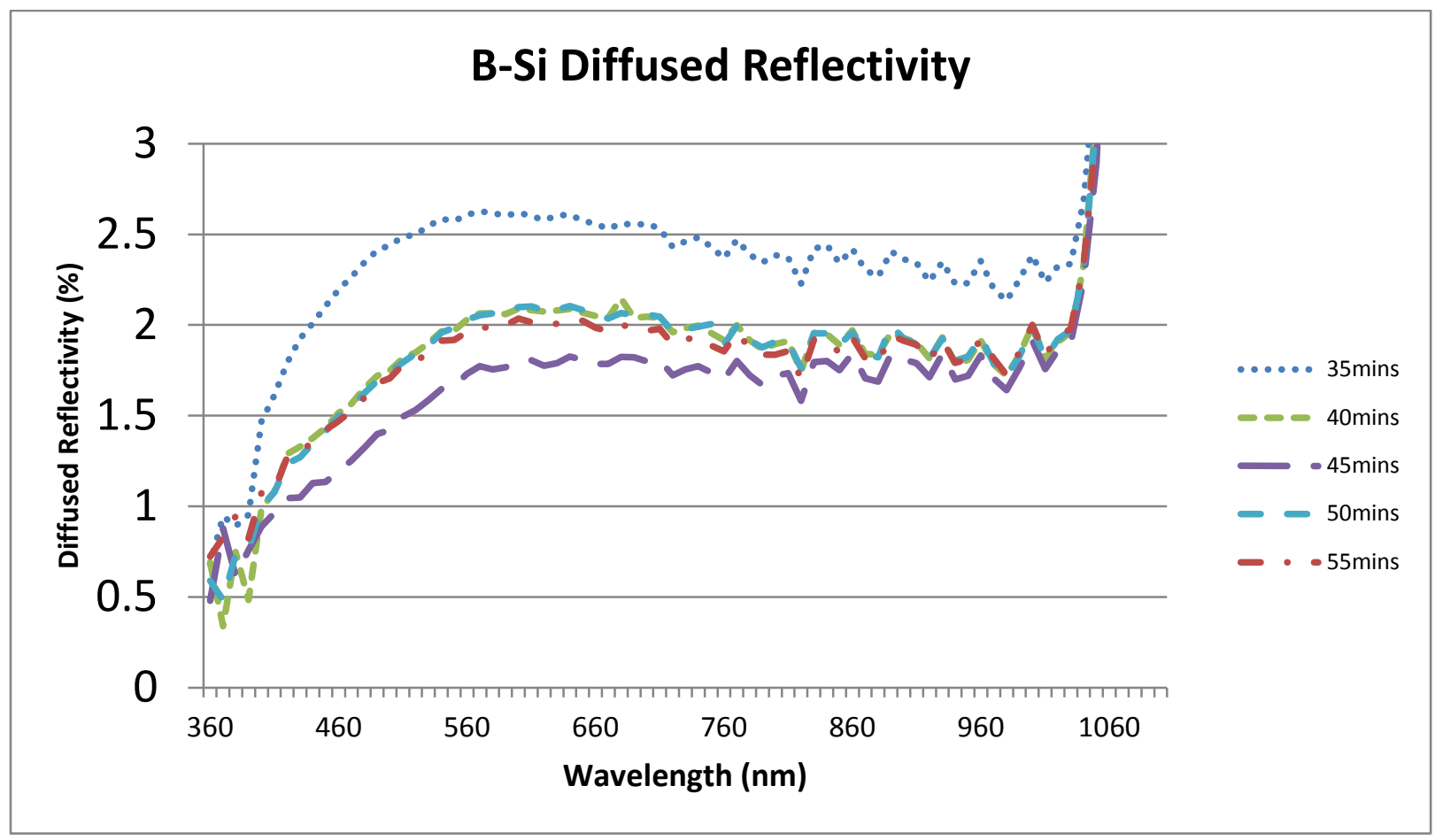

Figure 5-18: Diffused reflectivity measurement for b-Si solar cells with different etching times

Fig. 5-18 shows the diffused reflectivity of b-Si solar cells with different etching times. Here, the diffused reflectivity was measured by comparing differences between the output powers from the light source to the substrate and the received power from the substrate to the receiver in an integrated sphere system. The b-Si solar cell etched after 45 minutes had the lowest average diffused reflectivity at $1.9 \%$. The maximum diffused reflectivity of $2.63 \%$ was achieved at the shortest etching time, $35 \mathrm{~min}$. The diffused 
reflectivity decreased as the etching time increased until it reached 45 minutes. When the etching time is greater than 45 minutes, increasing the etching time increases the diffused reflectivity. Because the total surface reflectivity was dominated by the diffused reflectivity, the b-Si solar cell etched with an etching time of 45 minutes had the lowest total surface reflectivity compared to b-Si solar cells with other etching times.

At wavelengths above $1000 \mathrm{~nm}$, the diffused reflectivity of b-Si solar cells etched at different times rapidly increased to $100 \%$. This is caused by the fact that the photons in the incident light with energy less than the bandgap energy could not be absorbed by the c-Si substrate. Due to limitations of the measurement system, the diffused reflectivity measured at wavelength below $360 \mathrm{~nm}$ was all from the outside environment. The experiment results at this region were not used for evaluation even though it showed in the c-Si absorption spectrum.

\subsubsection{Effect of Fabrication Process on b-Si Structures}

A major advantage of $b-\mathrm{Si}$ solar cells with needle-shaped structures compared to those with other structure shapes is that the total reflectivity can be designed to have a very low level. Based on the total reflectivity measured in previous sections, the b-Si solar cell etched for 45 minutes with needle-shaped structures had the lowest surface reflectivity at $1.9 \%$. However, with a large height to width aspect ratio, the needle-shaped structures had less physical strength compared to structures with other shapes. In order to identify the strength of the needle-shaped structures, the effect of fabrication processes on needleshaped structures was investigated. The standard RCA cleaning process was used to clear the substrate from environment contamination before every fabrication step. The photoresist layer deposition is a necessary process for solar cell fabrication for contact 
formation. These two fabrication processes would have large effects on the needle-shaped structures since both of them involve aqueous solutions in physical contact with the nanostructures.

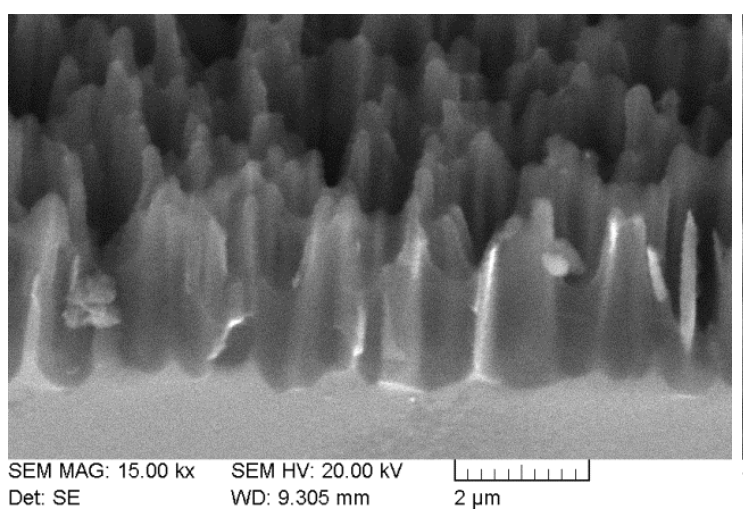

(a)

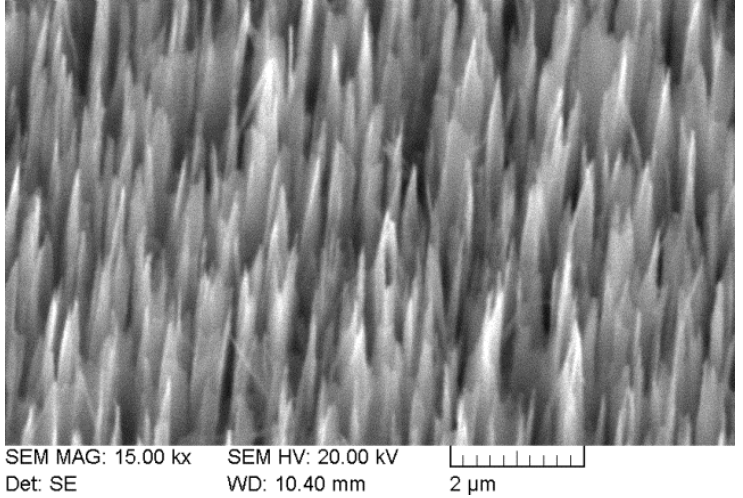

(b)

Figure 5-19: SEM images for b-Si nanostructures after (a) photoresist layer deposition and (b) standard RCA cleaning process

Fig. 5-19 shows SEM images of a sample b-Si solar cell's top surface, where (a) is after a photoresist layer deposition and (b) is after a standard RCA cleaning process. The two b-Si solar cells used in the experiment were etched for 45 minutes. Based on the SEM image in Fig. 5-19 (a), the layer deposited on the needle-shaped structures did not form a uniform layer around the structures. The photoresist layer created a bridge layer at the empty space between the adjacent needle-shaped structures. The needle-shaped nanostructures did not seem to have any damages from the deposition process since the shape of the structures before and after the deposition remained the same. According to Fig. 5-19 (b), the needle-shaped structures existed after the standard RCA cleaning process as the b-Si structures maintained their original needle-like shape. Since the deposition process and the cleaning process were not reactive with c-Si material, the geometry of b-Si structures were not changed by these two fabrication processes. 


\subsubsection{PL Measurement}

Due to the factor that the ECR etching system has been broken since June 2014, we only had a few b-Si samples available for investigation. Since we were unable to fabricate additional b-Si solar cells for experiments, a new method needs to apply to the b-Si samples to demonstrate their optical properties. The purpose of a photoluminescent (PL) measurement is to demonstrate the optical properties of b-Si solar cells without completing the whole solar cell fabrication procedure. The intensity of PL represents the intensity of photons absorbed by the substrate surface. Since we know that, a substrate with a high surface recombination will have higher photon absorption compared to a substrate with a low surface recombination. Therefore, we can speculate the level of surface recombination of the two samples by comparing their PL intensity measurement results.

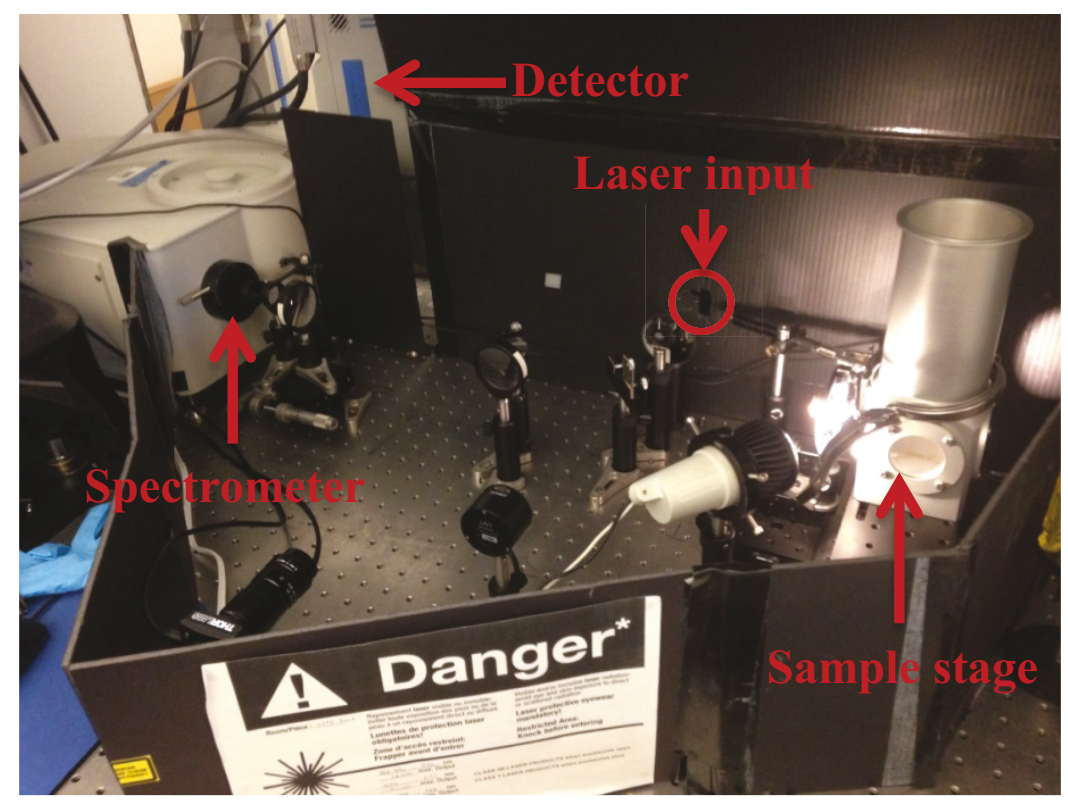

Figure 5-20: Photo image of the PL measurement system in the SUNLAB 
We conducted the PL intensity measurement in the SUNLAB at the University of Ottawa. Fig. 5-20 shows the setup of the PL measurement system built inside the SUNLAB. The PL was excited by a continuous wave (CW) diode-pumped solid state (DPSS) laser of approximately $350 \mathrm{~mW}$ at $532 \mathrm{~nm}$. Before the laser reached the b-Si sample, a circular filter was placed on a laser path to adjust the incident power from the laser source to the sample. The b-Si samples were placed on a sample stage inside a small alloy vacuum to control the measurement temperature. The PL signal from the sample was collected by an imaging spectrometer with a $0.32 \mathrm{~m}$ focal length and a 600 lines $/ \mathrm{mm}$ grating. A mirror filter with the ability to block light with a wavelength less than $540 \mathrm{~nm}$ was placed via the path of the PL signal. This was used to reduce the effect from the diffused laser signal in the system caused by the high power laser source. The resulting emission was detected by a liquid nitrogen cooled silicon CCD from room temperature to $78 K$.

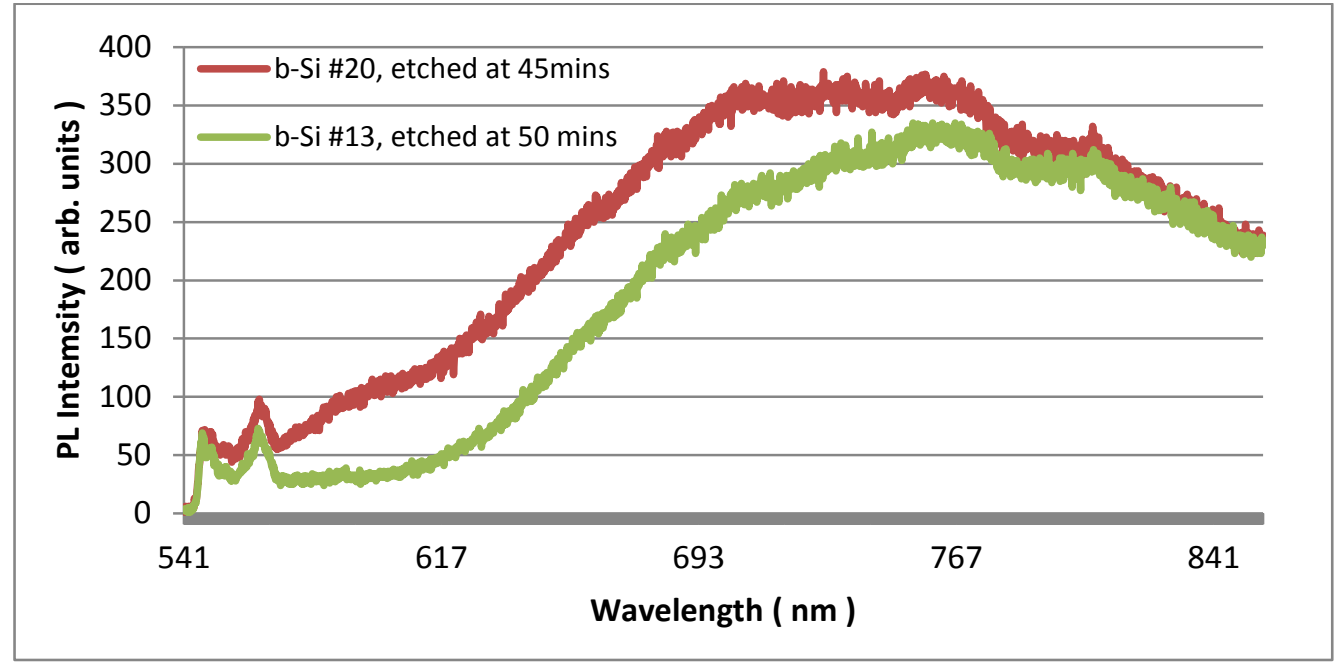

Figure 5-21: PL measurements obtained from b-Si samples formed by ECR at different etching times

First, we investigated the dependence of the b-Si PL on the ECR etching time. Two bSi samples involved in this experiment were etched by the ECR system for 45 minutes 
and 50 minutes, respectively. The PL signal from the b-Si samples was excited by a 350 $\mathrm{mW}$ power light at $532 \mathrm{~nm}$. Fig. 5-21 shows the PL signal measurements obtained from bSi samples formed by the ECR etching method for two different etching times. According to the PL results, the PL signal intensity of the b-Si sample decreased with increasing etching time from $560 \mathrm{~nm}$ to $780 \mathrm{~nm}$ wavelength. This is because the sample with longer etching time appears to have larger surface area compared to the sample with short etching time. Increased etching time of $b$-Si sample will increase the height and decrease the width. However, the magnitude of height alteration is larger than the magnitude of width alteration, which makes the height dominate the alteration of the surface area. According to Fig. 5-14 and Fig. 5-15, the difference in height between the samples etched after 45 minutes and 50 minutes was around $0.5 \mu \mathrm{m}$, which was approximately 5 times larger than the difference in width, around $0.11 \mu \mathrm{m}$.

Since the PL system measures the emission of light from a substrate under an optical excitation, the PL intensity investigations can be used to characterize the relative rates of radiative recombination on the substrate surface [63]. In this thesis, all the b-Si samples were fabricated from one c-Si wafer. The only difference between the two b-Si samples with different etching times was the height of b-Si structures. Because a material with a higher PL intensity has a higher light emission on the surface than that with a lower PL intensity, the b-Si sample etched for 45 minutes suffered less surface recombination than the b-Si sample with a longer etching time. A longer etching time contributed to a higher structure height, hence a larger height to width aspect ratio. As a result, the surface recombination effect increased with increasing surface area on the b-Si structures. The 
experiment results matched with the results from the theoretical design part in which the b-Si solar cell with a less etching time suffered smaller surface recombination effect.

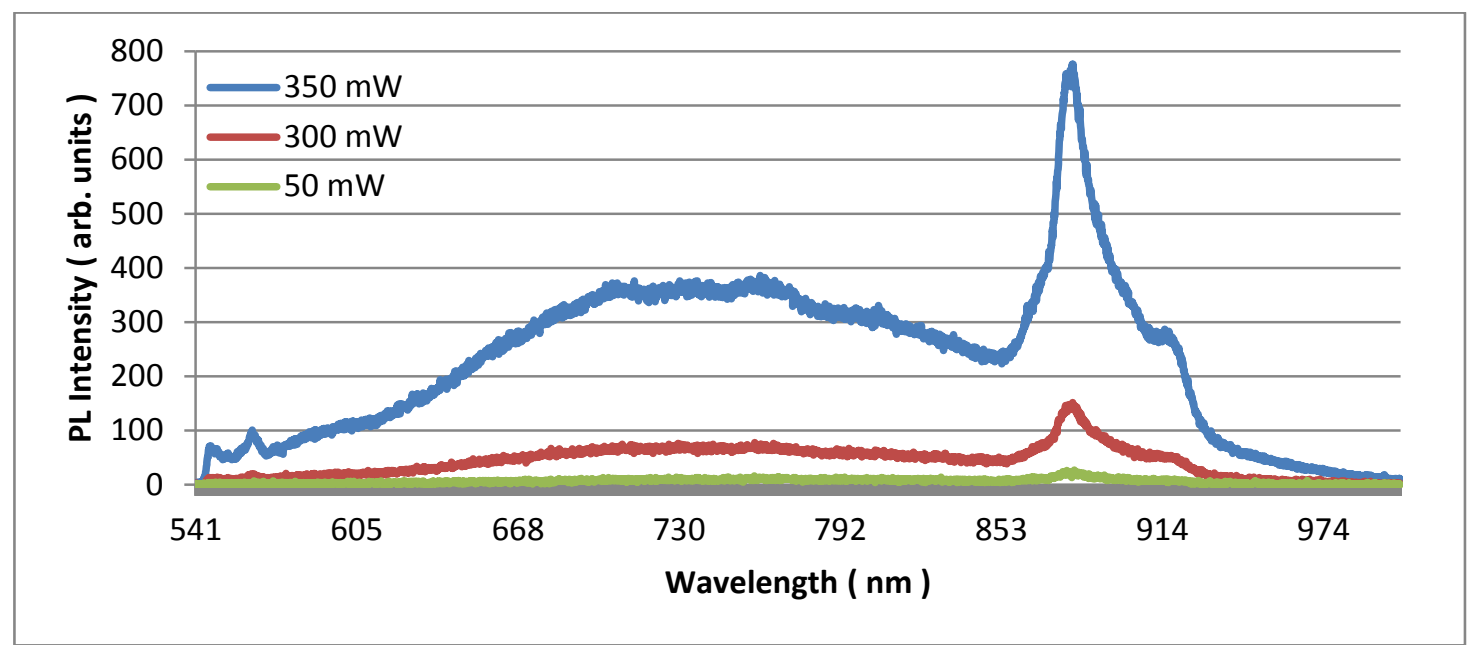

Figure 5-22: PL measurements obtained from b-Si sample with a 45 minute etching time at different excitation power levels

In addition, the influence of the incident power from the laser source to the b-Si sample on the PL measurement result was investigated. By adjusting the mirror filter placed between the laser source and the b-Si samples, the power of light excited from laser source was set at 350,300 , and $50 \mathrm{~mW}$, receptivity. Fig. 5-22 illustrates the PL measurement results obtained from the b-Si sample with different excitation power levels. As shown in the illustration above, the PL intensity from the b-Si sample decreased with decreasing excitation power. When the excitation power decreased from $350 \mathrm{~mW}$ to $300 \mathrm{~mW}$, an average of $60 \%$ decrease in PL intensity was observed across all wavelengths. The PL intensity for the b-Si sample using a $50 \mathrm{~mW}$ excitation power laser source was close to zero for most wavelengths. However, changing the excitation power did not significantly alter the trend of the PL intensity measurement. Two peaks in the PL intensity using a $350 \mathrm{~mW}$ excitation power were aligned to that using a $300 \mathrm{~mW}$ excitation power. 


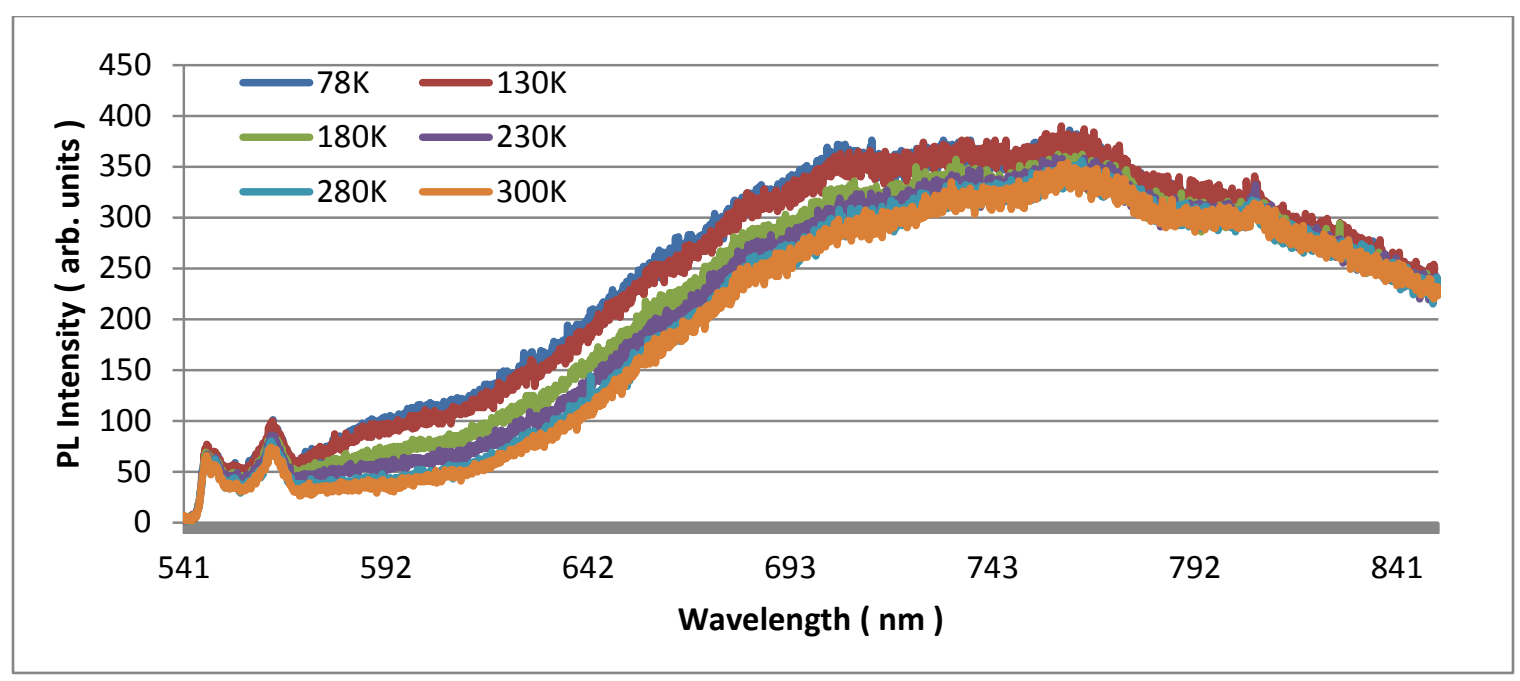

Figure 5-23: PL measurements obtained from b-Si sample with a 45 minute etching time at different temperatures

Moreover, we investigated the influence of the b-Si PL intensity at different temperatures. Liquid nitrogen was injected into the vacuum and used to reduce the temperature of the sample stage. Since the sample stage was made by copper, it was a perfect heat conductor, and it was able to adjust the temperature of b-Si samples placed on the top. A digital temperature detector was connected to the sample stage to monitor its temperature in real time. The excitation power of the laser source was set at $350 \mathrm{~mW}$, and the temperatures of the b-Si sample were set at 78, 130, 180, 230, 280, and $300 \mathrm{~K}$, respectively. The b-Si sample used in this experiment was etched for 45 minutes in the ECR system. Fig. 5-23 shows the PL spectra of the b-Si sample at different temperatures. Based on the PL measurement results, the PL intensity from the b-Si sample decreased with increasing temperature of the b-Si sample. The changes in PL intensity were easily identifiable between $560 \mathrm{~nm}$ and $840 \mathrm{~nm}$ wavelength ranges due to the accuracy of the PL measurement system; the difference of PL intensity at wavelengths above $840 \mathrm{~nm}$ wavelength was difficult to recognize from the PL spectra. The reason for this phenomenon could be caused by the decreased surface recombination velocity at low 
temperatures. Due to the low surface recombination at the low sample temperatures, the PL intensity was increased with increasing emission light from the b-Si sample. Hence, all major PL measurements in this thesis were measured at the lowest sample temperature, which was $78 K$ to enhance the accuracy of the PL measurement results.

In order to reduce the high surface recombination caused by larger surface areas and defects introduced by the b-Si structures on the front of the solar cells, a damage removal process using chemical materials was added to the fabrication procedure. A rapid thermal annealing (RTA) process was one of the damage removal processes used on the b-Si solar cell to eliminate the defects on the structures [64]. We need the RTA process to study the effect of the damage removal process of b-Si solar cells on surface recombination.

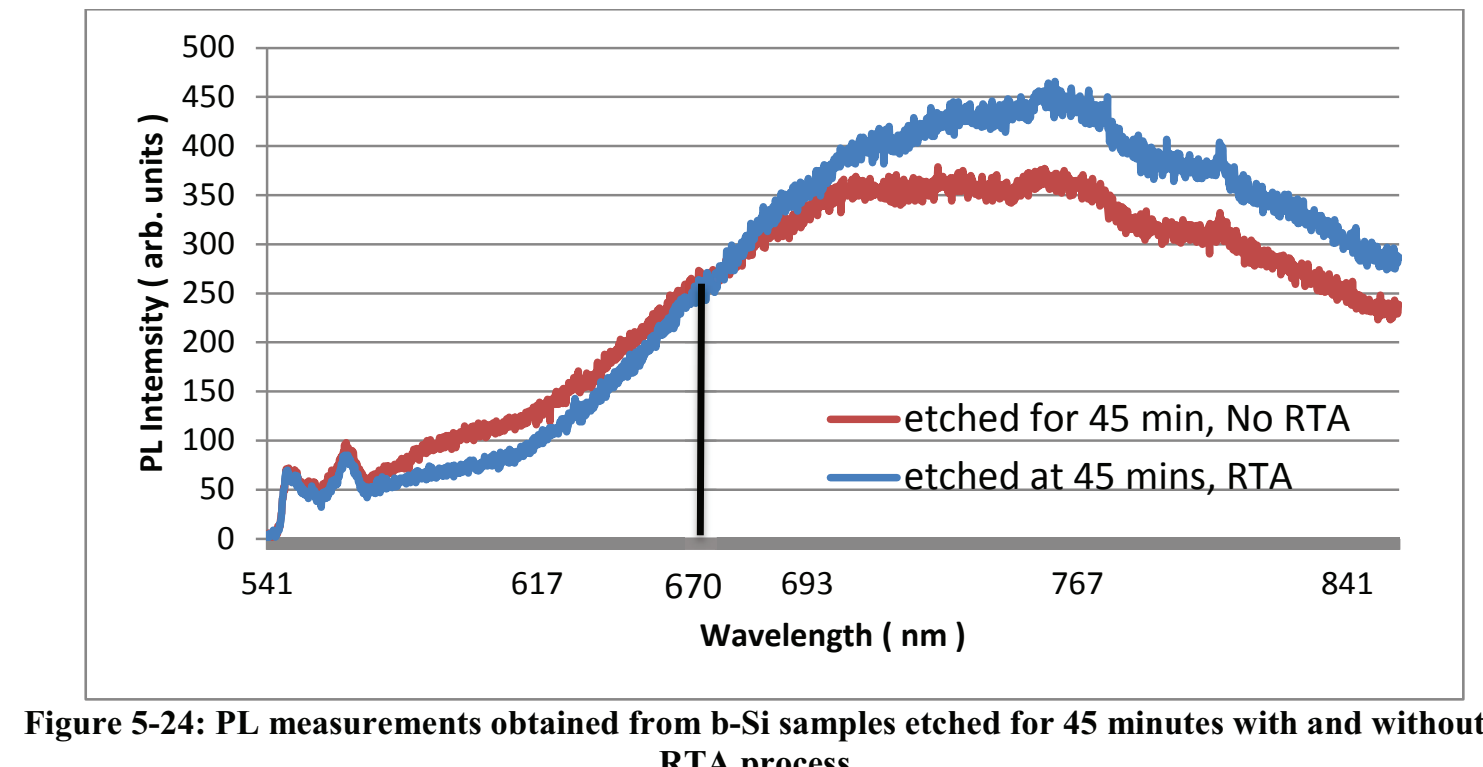
RTA process

The RTA process on a b-Si sample with an etching time of 45 minutes was fabricated in the CUMFF. The temperature of the RTA process was $1100{ }^{\circ} \mathrm{C}$, and the sample was annealed for $120 \mathrm{sec}$. After annealing, the b-Si sample was taken to the PL intensity measurements in the SUNLAB at the University of Ottawa. The excitation power of the $532 \mathrm{~nm}$ laser was $350 \mathrm{~mW}$, and the sample temperature was set to 78K. Fig. 5-24 
illustrates the PL intensity measurements of the b-Si samples with and without RTA process. According to the experimental results, the b-Si sample with the RTA process had a smaller PL intensity between $570 \mathrm{~nm}$ to $670 \mathrm{~nm}$ wavelengths and a larger PL intensity at wavelengths above $670 \mathrm{~nm}$. The smaller PL intensity occurred between $570 \mathrm{~nm}$ to 670 $n m$ wavelengths from the b-Si sample with the RTA process, which could be caused by the front diffusion corresponding to the RTA process. During the RTA process, a part of the front surface on the b-Si structures diffused further due to nitrogen $\left(\mathrm{N}_{2}\right)$ reaching high temperatures [65]. Consequently, more incident photons were absorbed by the b-Si sample. The emission of light in this wavelength range was smaller than the b-Si sample without the RTA process.

For light above $670 \mathrm{~nm}$ wavelengths, the PL intensity of the b-Si sample with RTA process was higher than that without the RTA process. This is due to the fast ramp-up and cool-down rate of the RTA that reduces the point defects on the needle-like shaped nanostructures, results in the lowest concentration of nonuniform defects, and limits the formation of defects by the migration process during the cooling down [66]. The defects on the b-Si sample surface were partially removed in the annealing even though the maximum temperature was below the melting point. Based on the experimental results, we found that the enhancement of PL intensity from the RTA process is larger than the decreased PL intensity caused by the RTA process. Hence, applying the RTA process on the b-Si sample is proven to reduce the surface recombination on the b-Si solar cells.

Since the unit of PL intensity measured is an arbitrary unit, the absolute value of the PL intensity is insignificant and thus not useful to demonstrate the performance of the bSi sample. However, the PL intensity measurements provide good guidance to optimize 
the surface recombination of the b-Si samples. According to the PL measurement results from different b-Si samples, the b-Si sample with a 45 minute etching time followed by a RTA process had the highest average and peak PL intensities among the samples. Based on the relationship between the PL intensity and the surface recombination, a b-Si solar cell with the same b-Si structures as this sample will suffer lower surface recombination and higher cell efficiency.

\subsubsection{Silicon Nanowires Solar Cell}

The horizontally-grown silicon nanowires (SiNWs) nanostructures were fabricated in collaboration with Professor Lyudmila Goncharova and her research group in the Ion Scattering and Nanophysics Laboratory at the University of Western Ontario. Prof. Lyudmila and her PhD student Ruiping grew SiNWs on the front of the substrates, and they also deposited Indium (In) adhesives on the back of c-Si substrate. Those fabrication processes took place on the substrates after the formation of the p-n junction at the CUMFF. Next, the substrates with the SiNWs were shipped back to the CUMFF to form the front metal finger grid and the back conductor. The intermediate steps and all other processing processes were all completed in the CUMFF. 


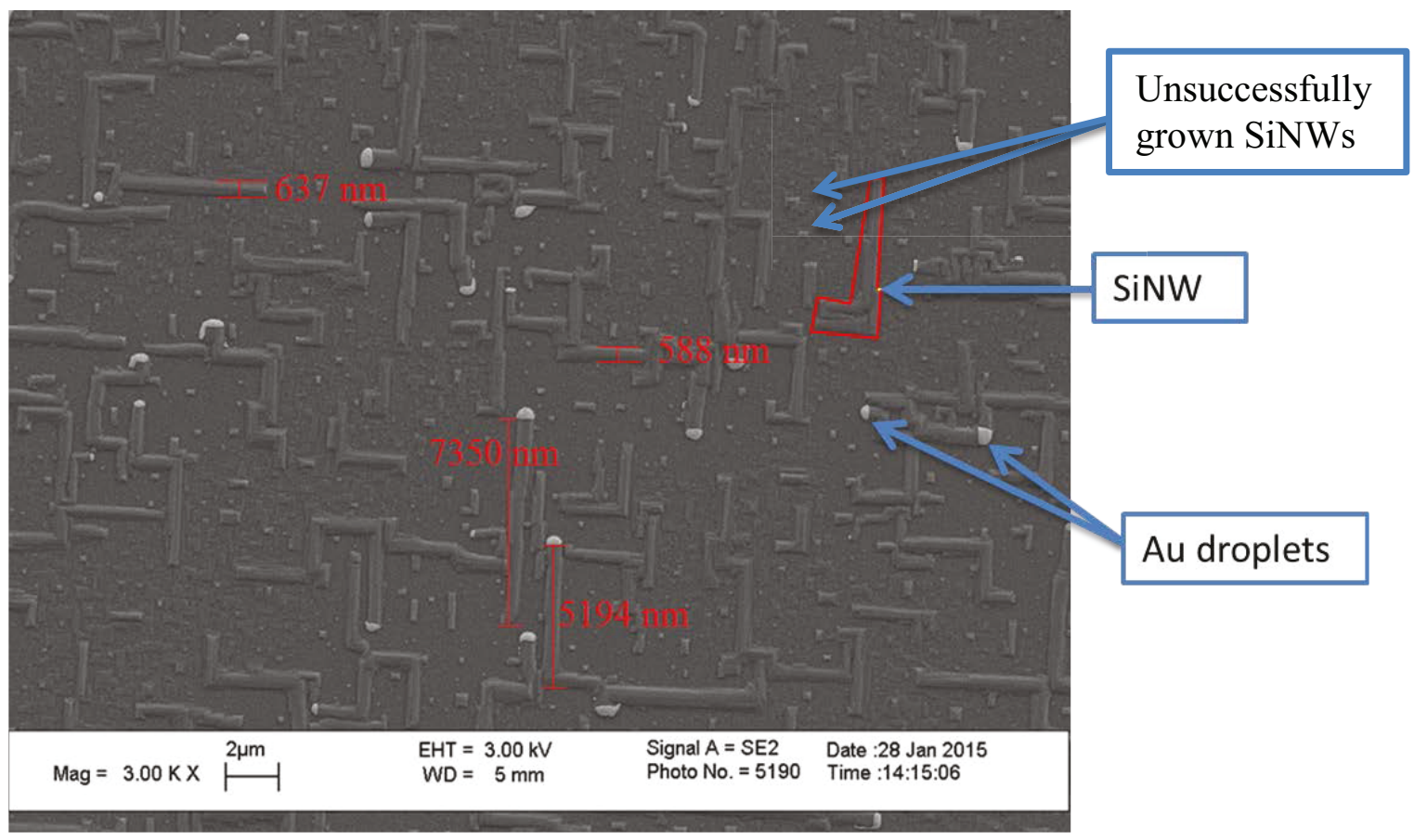

Figure 5-25: SEM top view image of c-Si solar cell with SiNW growth after 1 hour

Fig. 5-25 above shows a SEM top view image of SiNWs on c-Si solar cell with a 1 hour growth time. The nanostructure in the red surrounding area is a SiNW growth on the substrates. Two bright dots close to the SiNWs structures are gold ( $\mathrm{Au}$ ) seed droplets for the growth of the SiNWs. The average width of SiNWs after 1 hour growth time was approximately $600 \mathrm{~nm}$. The width of SiNWs was well controlled during the process due to the small disparity of SiNWs width. The length of SiNWs was difficult to calculate from the surface due to the "self-avoiding random walk" by the SiNWs via the growth process. The estimate value of SiNWs length was approximately $6 \mu \mathrm{m}$ based on the average length of the two measured SiNWs in Fig. 5-25.

Small silicon nanostructures were observed around the SiNWs in Fig. 5-25. Those were unsuccessfully grown SiNWs structures caused by the lack of etching time. During the growth process, silicon in the vapor phase impinges on a substrate covered with liquid 
Au droplets. Silicon atoms dissolve into the liquid droplets and excess silicon crystallizes as a solid wire at the droplet/substrate interface when their concentration in the droplet exceeds the saturation point. The growth time was an important parameter applied in the process because it affects the length of the SiNWs. When the growth time was short, the silicon atoms in the liquid droplets did not have enough concentration to exceed the saturation point. As a result, the liquid droplets with the silicon atoms were not able to generate solid wire at the interface and remained on the surface as the unsuccessfully grown SiNWs, which were the defects in the solar cells since they enhanced the surface recombination by increasing the surface area. Hence, the growth time of the SiNWs played an important role in the efficiency enhancement from the SiNWs.

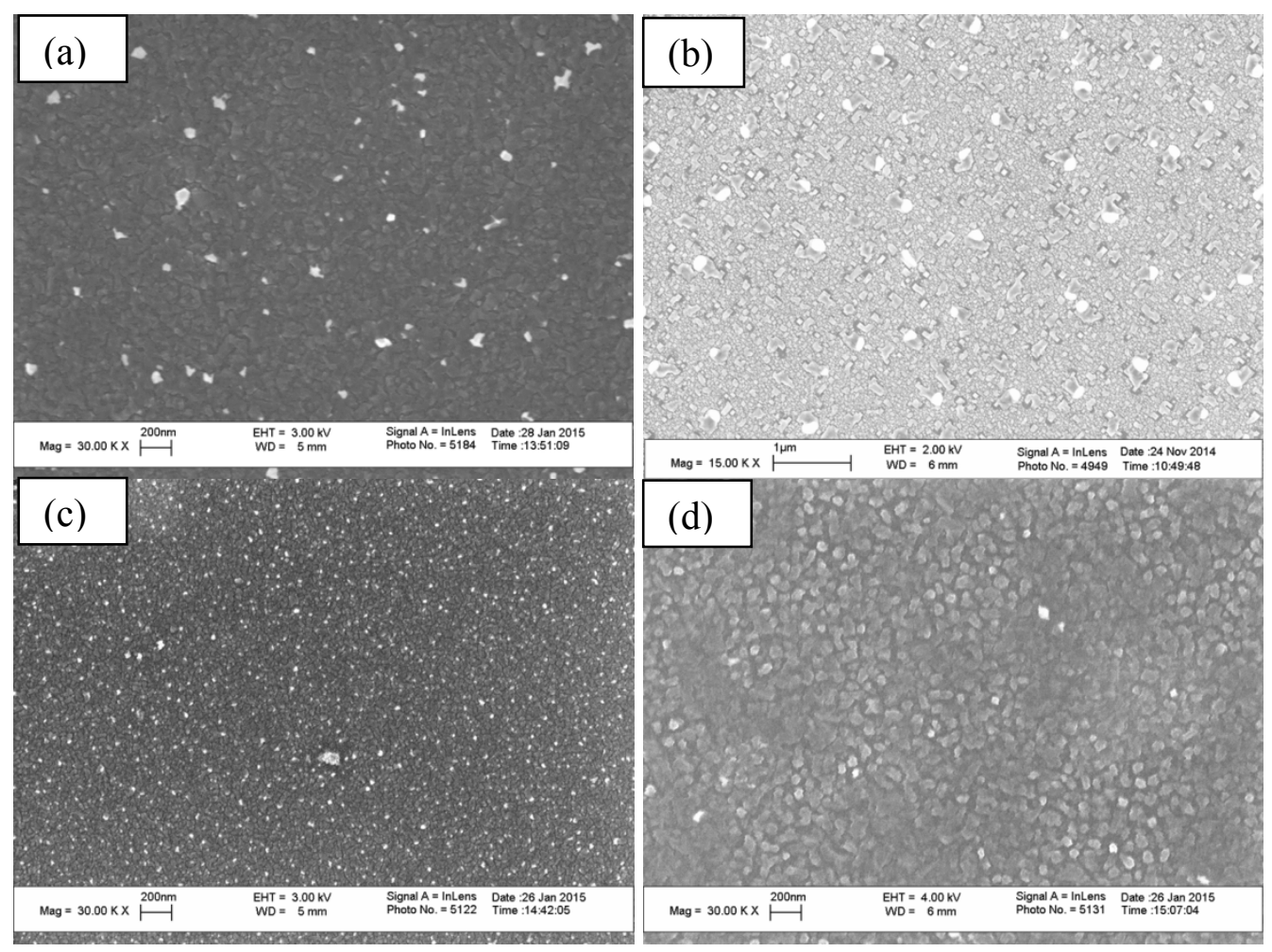

Figure 5-26: SEM images of SiNWs solar cell after growth time: (a) 0.5 hours (Sample \#15), (b) 1 hour (Sample \#4), (c) 1 hour (Sample \#8) and (d) 2 hours (Sample \#9) 


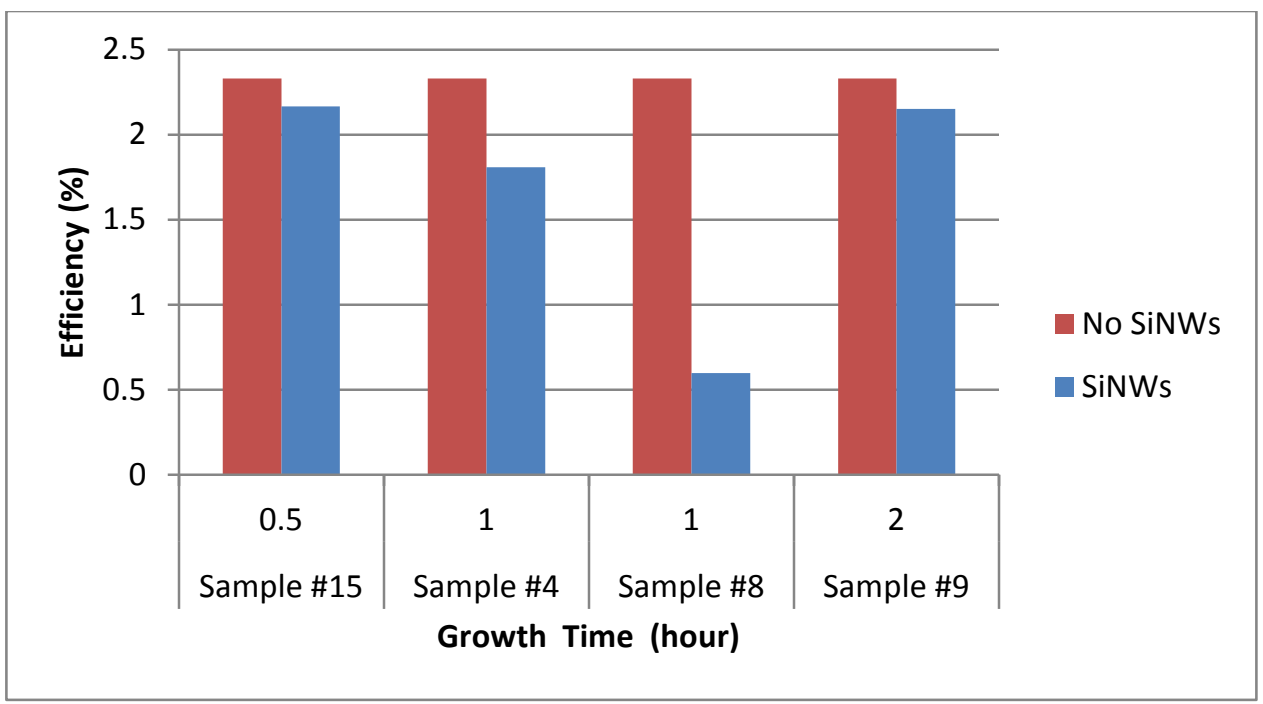

Figure 5-27: Efficiency measurements of SiNWs solar cell with different growth time

Fig. 5-26 shows the SEM images of unsuccessfully grown SiNWs on the c-Si solar cell, while Fig. 5-27 shows the efficiency measurements of c-Si solar cells with unsuccessfully grown SiNWs on the top. As designed in the simulation chapter, the SiNW growth on the c-Si solar cells was a type of surface textured nanostructure with a wire shape. However, the SiNW growth on these four solar cell samples appears to retain the droplet-shape after the growth process. The droplets involved with silicon atoms did not crystallize as solid wires at the droplet/substrate interface due to the lack of growth time. Hence, the SiNW growth on the top of c-Si solar cell with a growth time of less than 2 hours will not be able to form the wire-shaped structures.

According to the results, all four solar cells appeared to have a negative efficiency enhancement due to the droplet-shaped nanostructures instead of the wired shape. The average efficiency decrease from the SiNWs was $27.8 \%$ compared to the original solar cell without the SiNWs. Hence, in order to enhance efficiency from the SiNWs, the growth time of SiNWs has to be greater than 2 hours, and more experimental researching should be carried out to analyze the effect of growth temperature. 


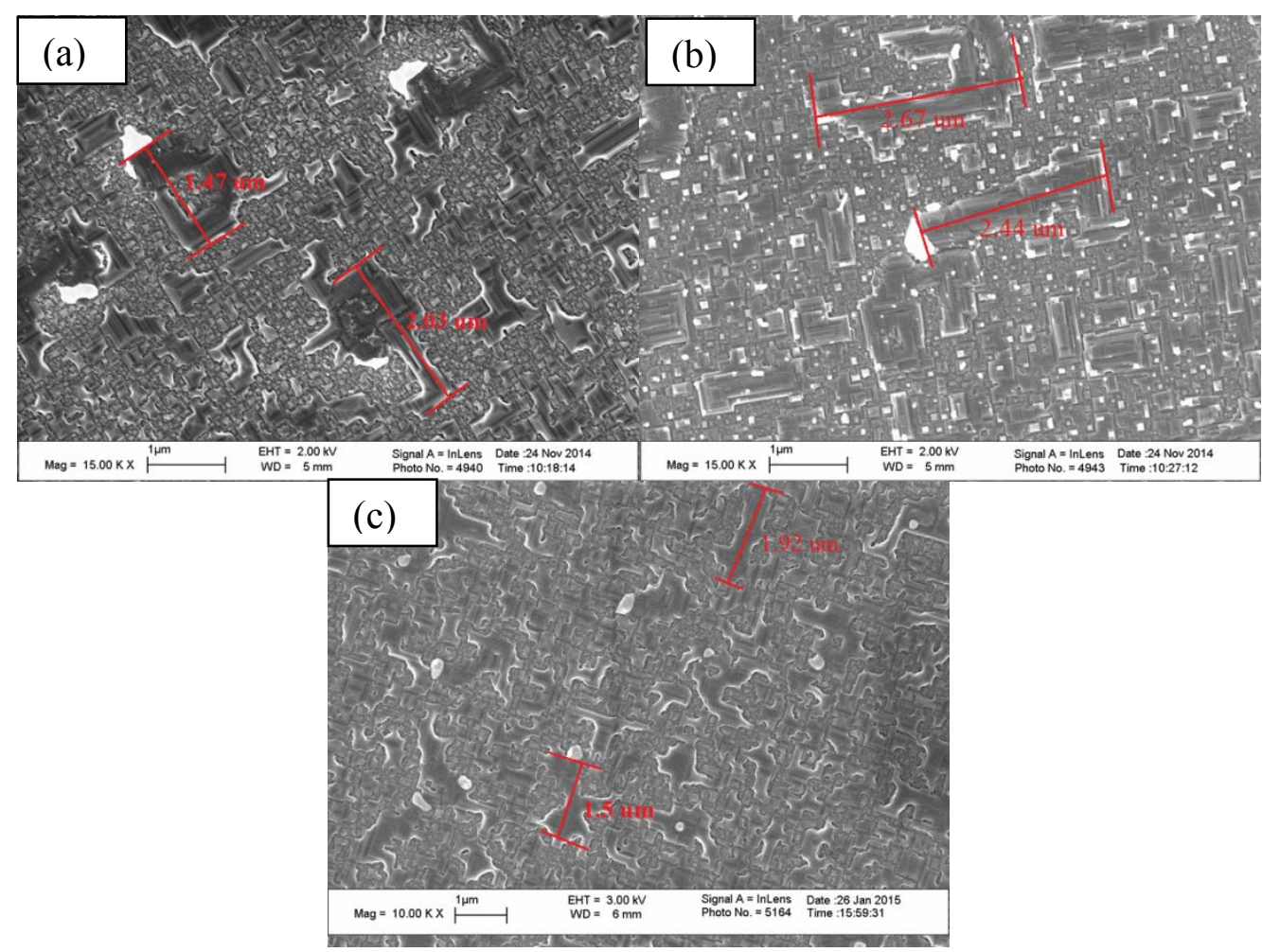

Figure 5-28: SEM images of SiNWs with irregular-shapes on c-Si solar cell after 4 hours of growth time: (a) $1.71 \mu \mathrm{m}$ (Sample \#12), (b) $1.75 \mu \mathrm{m}$ (Sample \#1) and (c) $2.55 \mu \mathrm{m}$ (Sample \#2)

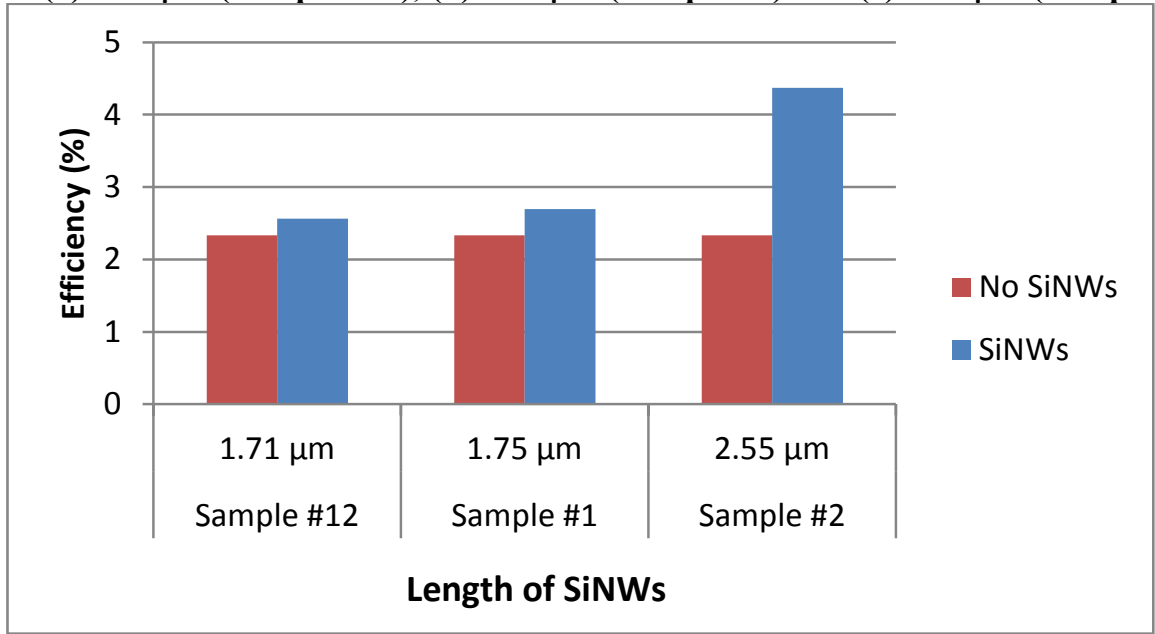

Figure 5-29: Efficiency measurements of c-Si solar cell with irregular-shaped SiNWs

Fig. 5-28 shows the SEM images of SiNWs with irregular shapes on the c-Si solar cell;

(a) is the image of sample \#1 and (b) is the image of sample \#2, while Fig. 5-28 (c) was

the image of sample \#12. Fig. 5-29 shows the efficiency measurements of c-Si solar cells

with irregular shaped SiNWs. According to Fig. 5-28, the average length of SiNWs on 
sample \#1 was approximately $1.75 \mu \mathrm{m}$, the average length of SiNWs on solar cell sample \#2 was approximately $2.55 \mu \mathrm{m}$, and the average length of SiNWs on solar cell sample \#12 was approximately $1.71 \mu \mathrm{m}$. The solar cells with irregular shaped SiNWs had enhanced cell efficiencies compared to the original solar cell. The efficiency of sample \#1 was $2.7 \%$, which had a $15.7 \%$ efficiency enhancement compared to the original cell. The efficiency of sample \#2 was $4.37 \%$, which had an $87.55 \%$ efficiency enhancement compared to the original cell. The efficiency of sample \#12 was $2.56 \%$, which had a $10.08 \%$ efficiency enhancement compared to the original cell. The sample with the longest SiNWs had the highest cell efficiency enhancement. According to Fig. 5-28, the sample with longer SiNWs appeared to have a higher efficiency enhancement than that with shorter SiNWs. The efficiency enhancement was related to the length of SiNWs when they had the same growth time.

The time of growth was 4 hours for all of these three samples. However, the shape of the SiNWs was different and irregular. There were two reasons: first and most importantly, the SiNW growth closely resembled a "self-avoiding random walk" in two directions on the substrate. The growth process was not well controlled due to the growth method and the limitation of fabrication facilities. The second reason was that the shape of SiNWs probably affected by the other parameters of fabrication, like temperature of growth. Due to the number of the SiNWs samples available for investigation, a directly relationship between the temperature of growth and the shape of the SiNWs has not yet been demonstrated in this thesis.

Due to the long growth time, the surface coverage of SiNWs on these three samples was high. Almost $100 \%$ of the surface area was covered by the SiNWs on sample \#2. 
Sample \#2 had the highest efficiency enhancement not only because the 4 hour growth time, but also the longest SiNW length of approximately $2.55 \mu m$. Longer SiNWs length will increase the surface coverage, hence reducing the surface reflectivity. Therefore, the surface reflectivity of the Sample \#2 was smaller than the reflectivity from the other two samples due to the highest efficiency enhancement. The shape of SiNWs had minor impact on the efficiency enhancement since sample \#2 had shapes closer to a wire-shape than the other two samples. Based on Fig. 5-28 and Fig. 5-29, the growth time for SiNWs was a key to the efficiency enhancement because it determined the length of SiNWs and the percentage of surface coverage. With enough growth time, the SiNWs on the front of c-Si solar cell can enhance the cell efficiency by reducing the surface reflectivity.

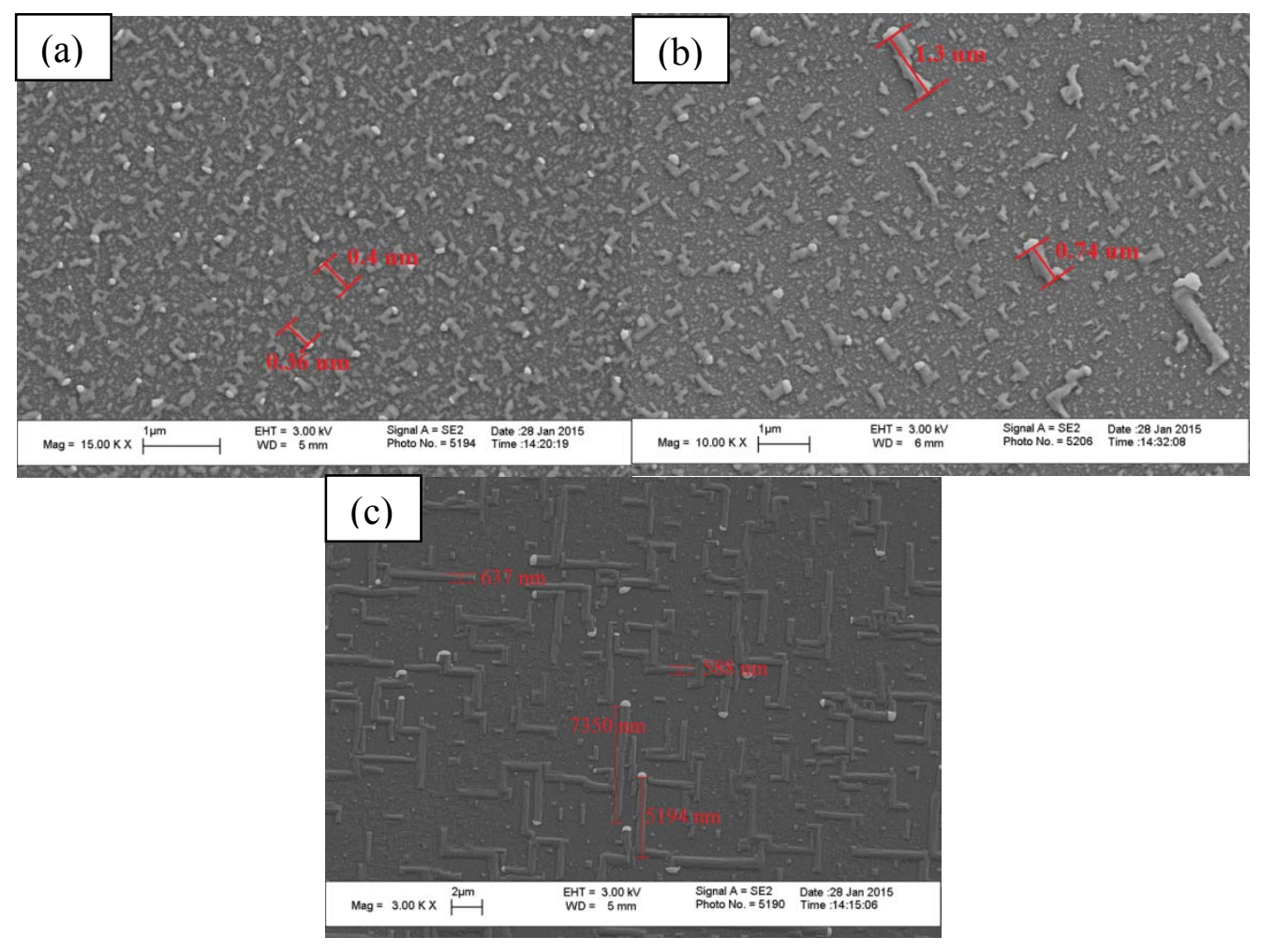

Figure 5-30: SEM images of SiNWs on c-Si solar cell after 1 hour growth time: (a) $0.38 \mu \mathrm{m}$ (Sample \#17), (b) $1.02 \mu \mathrm{m}$ (Sample \#14), (c) $6.27 \mu \mathrm{m}$ (Sample \#16) 


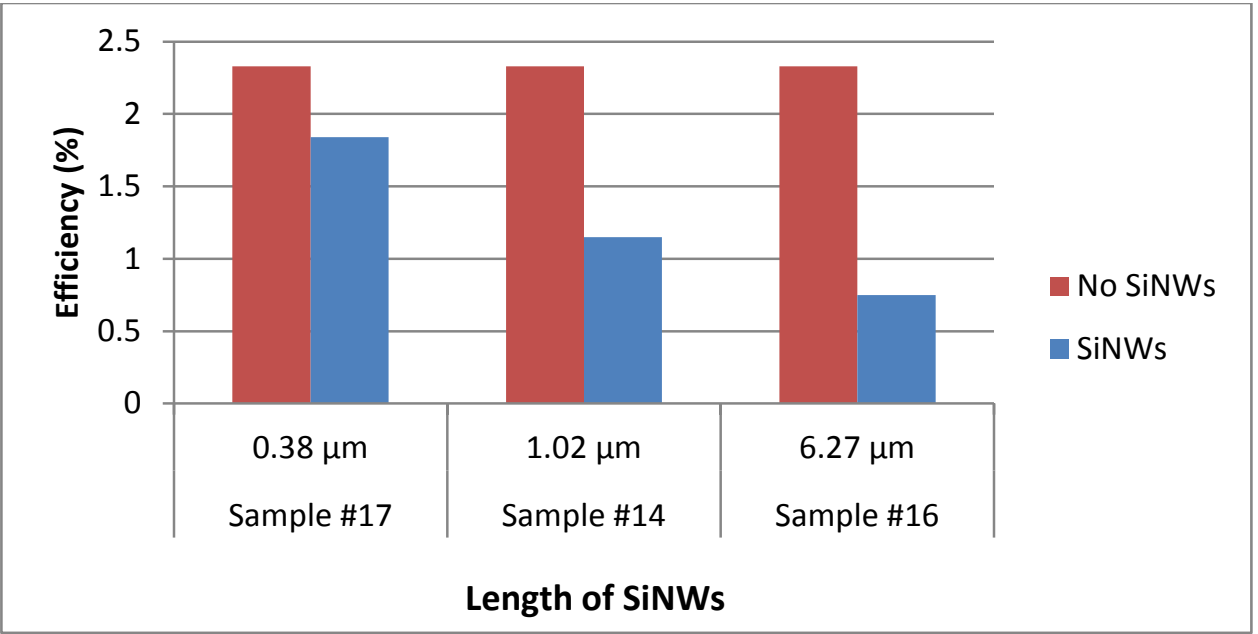

Figure 5-31: Efficiency measurements of c-Si solar cell with wire shaped SiNWs after 1 hour growth time

Fig. 5-30 shows the SEM images of SiNWs after 1 hour growth time on the c-Si solar cell; (a) the Sample \#17 had SiNWs with a length of $0.38 \mu m$; (b) sample \#14 with 1.02 $\mu m$, and (c) sample \#16 with a length of 6.27 $\mu m$. Fig. 5-31 shows the efficiency measurements of c-Si solar cells with wire-shaped SiNWs after 1 hour growth time in the order of length. According to Fig. 5-31, the SiNW solar cell with the longest length had the lowest cell efficiency. This observation disagrees with the demonstrations from the previous experiment that the sample with the longest SiNWs had the highest efficiency. The reason of the low efficiency is that the sample with the longest SiNWs had the lowest surface coverage compared to others. 


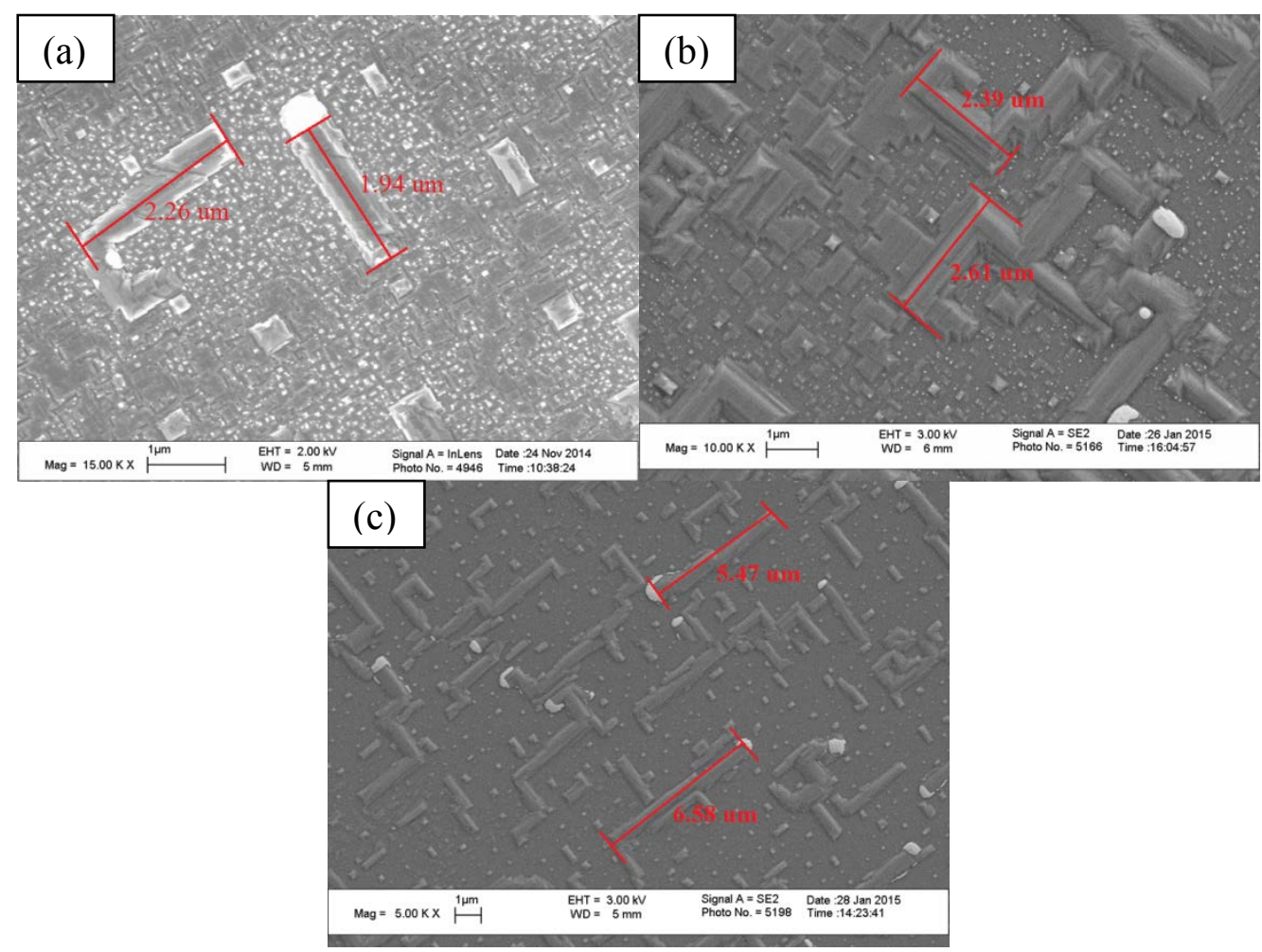

Figure 5-32: SEM images of SiNWs on c-Si solar cell after 2 hours growth time: (a) $2.1 \mu \mathrm{m}$ (Sample \#3), (b) $2.5 \mu \mathrm{m}$ (Sample \#13), (c) $6.02 \mu \mathrm{m}$ (Sample \#11)

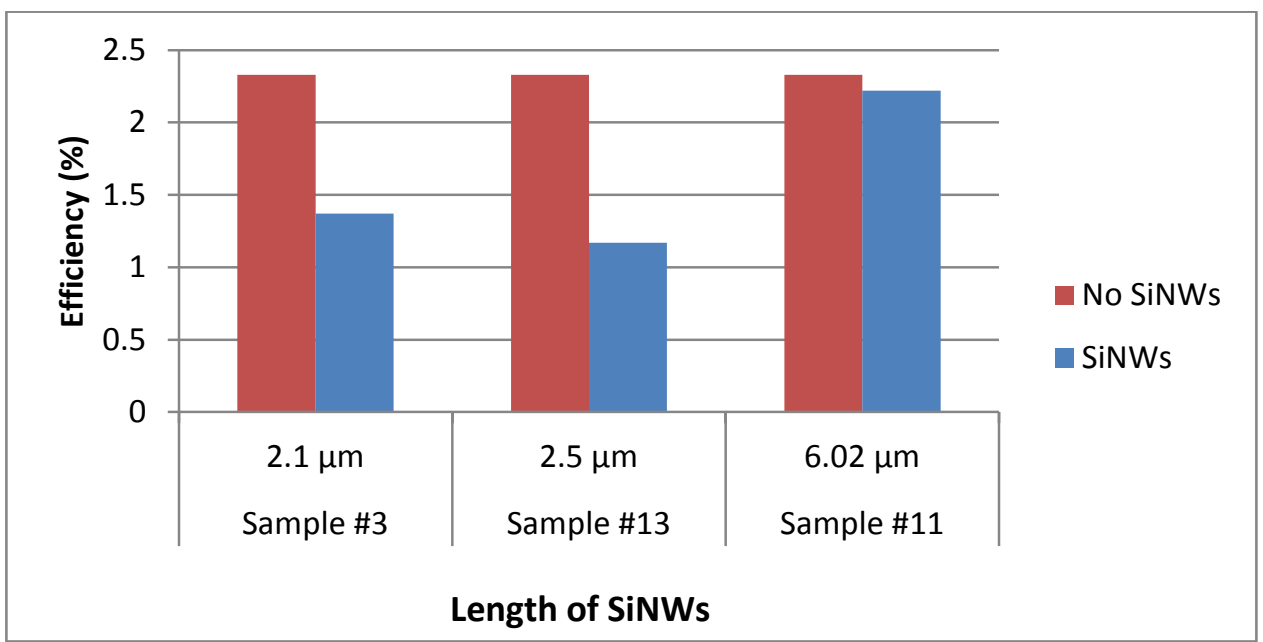

Figure 5-33: Efficiency measurements of c-Si solar cell with wire-shaped SiNWs after 2 hours growth time

According to Fig. 5-32, the average length of wire-shaped SiNWs after the 2 hours growth time on samples \#3, \#13 and \#11 was 2.1, 2.5, $6.02 \mu \mathrm{m}$, respectively. According to Fig. 5-33, the SiNWs solar cell with the longest length had the highest cell efficiency. 
This observation agrees with the results from the SiNWs solar cells after 4 hours growth time that the efficiency enhancement was related to the length of the SiNWs. At the same growth time, the SiNWs solar cell with the longer length had higher cell efficiency than that with the shorter SiNWs. However, all SiNWs solar cells after 2 hours growth time had the negative efficiency enhancement even with the longest length.

The reason of the negative efficiency enhancement is that no sample had a growth time longer than 2 hours. The solar cell samples with irregular SiNWs had efficiency enhancement since all of them had 4 hours growth time. The length of SiNWs on the sample with the highest efficiency enhancement was $2.55 \mu \mathrm{m}$, which was the longest length in the 4 hour group. However, the solar cell with $6.27 \mu \mathrm{m}$ SiNW length still had a negative efficiency enhancement. The length of SiNWs did not show a direct relationship to the efficiency enhancement. We conclude that only the growth time had a major influence on the efficiency enhancement from the SiNWs.

\section{Effect of Growth Time on Reflectivity}

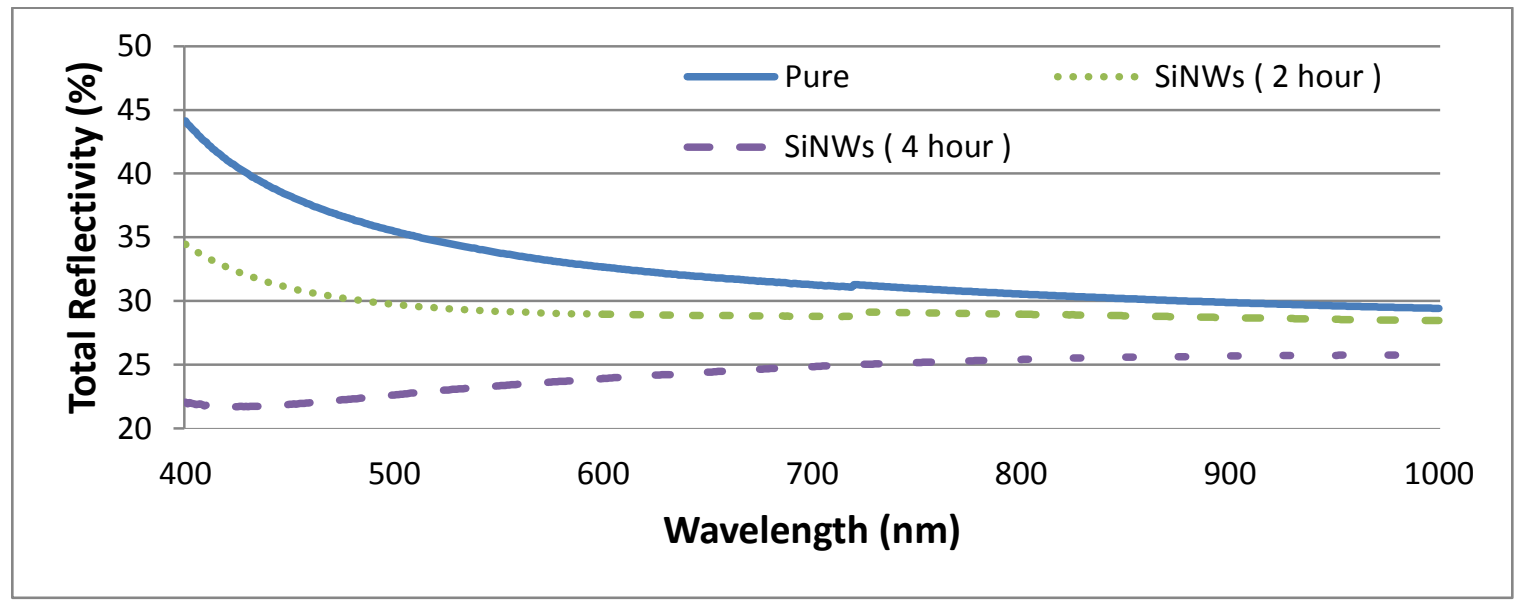

Figure 5-34: Total reflectance measurement of c-Si solar cell with and without SiNWs

Fig. 5-34 above shows the total reflectivity measurement results for a reference cell, a solar cell with a 2-hour growth time of SiNWs and a SiNWs solar cell with a 4-hour 
growth time. According to Fig. 5-34, the average total reflectivity of the pure c-Si solar cell, the solar cell with SiNWs growth after 2 hours, and the solar cell with SiNW growth after 4 hours was $30 \%, 28 \%$, and $26 \%$, respectively. At a $400 \mathrm{~nm}$ wavelength, the total reflectivity of the pure c-Si solar cell, the solar cell with SiNW growth after 2 hours, and the solar cell with SiNW growth after 4 hours was $44 \%, 34 \%$, and $22 \%$, respectively.

Based on the reflectivity measurement results, the solar cell with SiNW growth after 4 hours had the lowest average reflectivity and the lowest reflectivity at the $400 \mathrm{~nm}$ wavelength. The average reflectivity of SiNWs with a 4-hour growth time was $5 \%$ less than the SiNWs solar cell with a 2-hour growth time; this result matched the results from Fig. 5-29, 31, and 33: the solar cell with a longer growth time had less surface reflectivity due to a longer length and a larger surface coverage. Because the absorption spectrum of c-Si solar cell was from $400 \mathrm{~nm}$ to $1100 \mathrm{~nm}$ wavelength, the SiNWs solar cell with a 4hour growth time had $22 \%$ reflectivity at the $400 \mathrm{~nm}$ wavelength, which only equals half of the reflectivity of the pure c-Si solar cell. The SiNW solar cell with a 2-hour growth time also had decreased reflectivity at the $400 \mathrm{~nm}$ wavelength compared to the pure solar cell. Hence, the SiNWs growth on a c-Si solar cell will reduce the reflectivity across the entire absorption spectrum and perform better at short wavelengths. We conclude that the reduced surface reflectivity from the SiNWs depends on the growth time of SiNWs and the SiNWs with a 4-hour growth time have the lowest surface reflectivity. 


\section{Effect of Growth Time on Efficiency Enhancement}

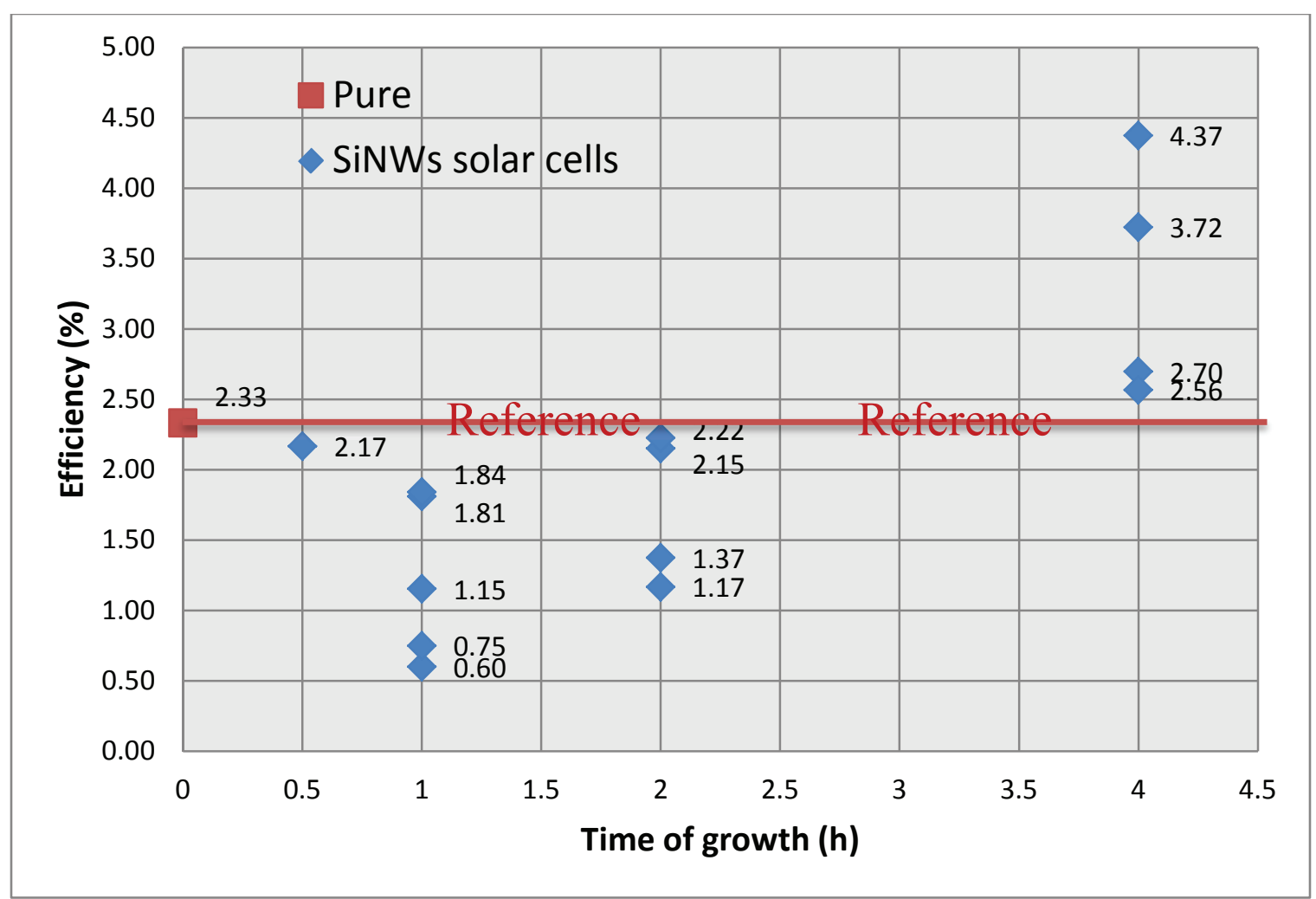

Figure 5-35: Efficiency measurements of c-Si solar cell with different growth times of SiNWs

Fig. 5-35 shows the efficiency measurements of c-Si solar cells with SiNW growth at several differing times. The red square in Fig. 5-35 represents the efficiency of a pure cSi solar cell, which was 2.33\%. The blue diamond in Fig. 5-35 represents the efficiency of solar cells with SiNW growth after different times. According to Fig. 5-35, one out of the 14 SiNWs solar cells had a 0.5 -hour growth time, and its efficiency was $2.17 \%$, which was a $7 \%$ negative efficiency enhancement. Five out of 14 SiNWs solar cells had a 1-hour growth time, and the highest efficiency was $1.84 \%$, which was a $21 \%$ negative efficiency enhancement. Four out of 14 SiNWs solar cells had a 2-hour growth time, and the highest efficiency was $2.22 \%$, which had a $4 \%$ negative efficiency enhancement. Four out of 14 SiNWs solar cells had a 4-hour growth time, and the highest efficiency was $4.37 \%$, which had an $87 \%$ efficiency enhancement. 
All SiNWs solar cells with a 4-hour growth time had the efficiency enhancement compared to the original cell, and the average efficiency enhancement was $43 \%$. There were two reasons: firstly, they had the highest surface coverage due to the irregular shaped surface structures; and secondly, they had the lowest surface reflectivity. No SiNW solar cells with a growth time less than 4 hours showed an efficiency enhancement. The SiNWs solar cells with a 2-hour growth time had higher average efficiency compared to the ones with a 1-hour growth time. This was due to the increased length of the SiNWs and the surface coverage. And this also proved that the size of the SiNWs depends on the growth time, which had the major effect on the efficiency enhancement.

We conclude that the SiNWs solar cells with a longer growth time have higher efficiency enhancement. The SiNWs solar cells with a growth time of 4 hours had an average efficiency enhancement equal to $43 \%$. The highest efficiency enhancement was $87 \%$ from solar cell sample $\# 2$ with a $4.37 \%$ efficiency.

\subsection{Experimental Results Summary}

We first demonstrated the effect of Ag MNP size on cell efficiency. The cubic Ag MNPs with three different sizes, $45 \mathrm{~nm}, 75 \mathrm{~nm}$, and $80 \mathrm{~nm}$, were directly deposited on the c-Si solar cells. The $45 \mathrm{~nm}$ size cubic Ag MNPs deposited on the c-Si solar cell had a negative efficiency enhancement across all samples. Five out of eight solar cell samples had an average efficiency enhancement equal to $4.7 \%$ when the $75 \mathrm{~nm}$ size cubic $\mathrm{Ag}$ MNPs deposited on the solar cell. Further increasing the size of MNPs increases the efficiency enhancement from the MNPs. Three out of five solar cell samples had an efficiency enhancement after depositing the $80 \mathrm{~nm}$ size cubic Ag MNPs. After that, a 25 
$n m$ thick $\mathrm{SiO}_{2}$ sublayer was inserted between the MNPs and the solar cell to test the effect of a sublayer. All eight solar cell samples with $80 \mathrm{~nm}$ size cubic Ag MNPs on a 25 $n m$ thick $\mathrm{SiO}_{2}$ sublayer on the c-Si solar cell showed a 7\% average efficiency enhancement. The average enhancement was $7 \%$, and the maximum enhancement was $18.3 \%$.

The b-Si sample etched after 45 mins had the lowest diffused reflectivity, which was approximately $1.9 \%$. The total reflectivity of b-Si sample was dominated by the diffused reflectivity since the specular reflectivity was too small. In order to test the optical properties of b-Si sample, the PL intensity of the b-Si sample was tested at the University of Ottawa. The b-Si etched after 45 minutes had larger PL intensity than the b-Si etched after 50 mins, proving that the b-Si sample with less etching time suffered less surface recombination.

The SiNWs with a growth time of 4 hours had a lower total reflectivity than the SiNWs with a 2-hour growth time and the original solar cell. The average reflectivity between $400 \mathrm{~nm}$ and $1000 \mathrm{~nm}$ was $24 \%$ for the SiNWs with a 4-hour growth. Only the SiNWs solar cells with a 4-hour growth time had efficiency enhancement in the experiment. The maximum efficiency enhancement was $87 \%$ from the solar cell with SiNWs of $2.55 \mu \mathrm{m}$ in average length. The average efficiency enhancement from four SiNWs solar cells with a 4-hour growth time was $43 \%$. Table 1 summaries the most optimal experimental demonstrations of the solar cell devices fabricated in this thesis. 
Table 1: Summary of the most optimal experimental results

\begin{tabular}{|c|c|c|c|c|}
\hline Nanotechnology & $\begin{array}{c}\text { Specific } \\
\text { Application }\end{array}$ & \multicolumn{3}{|c|}{ Optimization } \\
\hline $\begin{array}{c}\text { Plasmonics } \\
\text { nanoparticles(MNPs) }\end{array}$ & $\begin{array}{c}\text { Cubic Ag } \\
\text { MNPs } \\
(\%)\end{array}$ & 4.85 & $\begin{array}{c}\text { Max }=18.3 \%, \\
\text { Average }=7 \%\end{array}$ & $\begin{array}{c}\text { Ag MNPs on on a 25 } \\
\text { nm thick SiO2 } \\
\text { layer }\end{array}$ \\
\hline $\begin{array}{c}\text { Surface-textured } \\
\text { nanostructures }\end{array}$ & $\begin{array}{c}\text { Black Silicon } \\
\text { (b-Si) }\end{array}$ & N/A & $\begin{array}{c}\text { Diffused } \\
\text { reflectivity }= \\
\sim 1.9 \%\end{array}$ & $\begin{array}{c}\text { Etched after 45 } \\
\text { minutes (best case) }\end{array}$ \\
\hline $\begin{array}{c}\text { Surface-textured } \\
\text { nanostructures }\end{array}$ & $\begin{array}{c}\text { Silicon } \\
\text { Nanowires } \\
\text { (SiNWs) }\end{array}$ & 4.37 & $\begin{array}{c}\text { Max }=87 \%, \\
\text { Average }=43 \%\end{array}$ & $\begin{array}{c}4 \text { hour growth time } \\
\text { (best case) }\end{array}$ \\
\hline
\end{tabular}




\section{Conclusions}

This thesis covers the analysis of two methods that will enhance the total cell efficiency of single crystalline silicon (c-Si) solar cells. The first method uses plasmonic nanotechnology with an array of plasmonic cubic metal nanoparticles (MNPs), and the second method employs the surface modification technology, where the needle-like black silicon nanostructures and the horizontally-grown silicon nanowires are utilized.

The plasmonic nanotechnology is based on the localized surface plasmon resonances (LSPRs) exhibited by the MNPs when light was illuminated. This resonant behavior is exploited for improving light trapping and photocurrent generation in the near field of the c-Si solar cells with reduced surface reflectance when the MNPs are deposited on the front surface.

The use of cubic Ag MNPs has been studied in great detail for the application of light trapping in c-Si solar cells. The use of cubic Ag MNPs differs from previous works in that most have made use of hemispherical Ag MNPs, which have a less involved fabrication process and lower costs, yet a small enhancement in efficiency. Cubic Ag MNPs have high scattering cross-sections, compared to spheres or hemispheres over a broad wavelength range, which provides extra enhancement in light trapping and increases the backward electric field intensity into the substrate. Cubic Ag MNPs enable broadband photocurrent enhancement, a major step towards the viable use of plasmonic MNPs for light trapping in c-Si solar cell applications.

The finite-difference time-domain (FDTD) method was used to perform all optical and physical simulations of the MNP properties. From this platform, extensive optimizations were performed to determine the best configuration for cell efficiency enhancement in 
terms of the particle shape, size, material, surface coverage, and sublayer thickness.

Increasing the cell efficiency up to $8 \%$ was shown for $80 \mathrm{~nm}$ size cubic Ag MNPs on a 25 $n m$ thick $\mathrm{SiO}_{2}$ sublayer compared with a mere $2 \%$ for hemispherical Ag MNPs.

Most fabrication was done at the CUMFF. Fabrication of all the c-Si solar cells was carried out with a cubic Ag MNP monolayer on the front surface to verify the simulation results. The c-Si solar cells used for the experiment were fabricated from p-type c-Si wafers. Moreover, the $\mathrm{SiO}_{2}$ sublayer was deposited by sputter deposition on the solar cells before the MNP deposition. The cubic Ag MNPs with a $40 \mathrm{~nm}$ in edge length deposited on a c-Si solar cell without a $\mathrm{SiO}_{2}$ sublayer displayed an average of $12 \%$ decrease in cell efficiency. An average increase of $2 \%$ in cell efficiency became evident when the cubic Ag MNPs with an average edge length of $75 \mathrm{~nm}$ were deposited without the $\mathrm{SiO}_{2}$ sublayer. An increase in cell efficiency of up to $7 \%$ was shown for cubic $\mathrm{Ag}$ MNPs with an average size of $80 \mathrm{~nm}$ on a $25 \mathrm{~nm} \mathrm{SiO}_{2}$ sublayer, compared to just $2 \%$ for the same particles without a sublayer. The experiment results showed less cell efficiency enhancement predicable by the simulations. This decrease in the enhancement was due to the non-uniform distribution of the cubic Ag MNPs and particle-stacking from the deposition method. The proposed enhancement method should be able to reach the simulated maximum enhancement of $8 \%$ on the $\mathrm{SiO}_{2}$ sublayer with more optimized parameters.

One of many advantages of the MNPs is their small size as all MNPs are in the nanometer scale. Only a very small amount of fabrication material is required to fabricate a large number of MNPs. In our experiments, the cost of silver nitride used for the synthesis of our MNPs was less than $0.5 \$$, which offered a sufficient surface coverage of 
a solar cell sample area of $20 \mathrm{~cm}^{2}$. And our deposition method is cheaper than other fabrication methods due to our simple synthesis process is purely chemical and can be used for solar cells with an area on the order of $10 \mathrm{~cm}^{2}$. The process was purely chemical and can be used for solar cells with an area on the order of $10 \mathrm{~cm}^{2}$. In fact, the most expensive part of the fabrication was to create the $p-n$ junction of the solar cell itself and the silicon wafer cost.

Next, the thesis investigated the surface modification technology for efficiency enhancement. The needle-like black silicon (b-Si) surface textured nanostructures on the front surface enable a reduction of the surface reflectivity to a low level compared to the conventional c-Si solar cell surface. Since the size of b-Si structures was smaller or closer to the wavelength of the incident light, the transition of the refractive index between the structures and the surrounding environment was small. Since the surface reflectivity depends on the refractive index difference between the two media, a small refractive index difference contributes to a small surface reflectivity.

The b-Si structures on the c-Si solar cell were etched using the electron-cyclotron resonance (ECR) ion etching method. The reaction gases used in the ECR method were $\mathrm{SF}_{6}$ and $\mathrm{O}_{2}$. A detailed fabrication procedure of $\mathrm{b}-\mathrm{Si}$ solar cell was explained in Chapter 4. The first step was to etch the needle-like structures onto the c-Si wafer. The damage removed etching (DRE) was the second step to remove the defective structures and to reduce surface recombination. Then, the c-Si wafer was diffused, followed by the deposition of the metal grid on the top surface. The height of needle-like structures was designed to be low so it would not influence the diffusion process and the surface metal conductor. The height of the needle-like b-Si nanostructures depended on the etching 
time and the reaction gas ratio during the etching process. Due to the limitation of the fabrication tool, successful etches of the b-Si structures only occurred when the gas ratio equaled to 0.5 . The c-Si wafer with a 45 minute etching time showed an average of $3 \mu \mathrm{m}$ in height for the needle-like b-Si nanostructures. After increasing the etching time to 50 minutes, the needles had increased to an average $3.5 \mu \mathrm{m}$ in height with an estimated etching rate of $0.1 \mu \mathrm{m} / \mathrm{min}$. The optimal height in this set of experiments is $3 \mu \mathrm{m}$ to ensure that the space under the b-Si structures could be efficiently diffused. An average of a $0.1 \%$ specular reflectance was measured with the $3 \mu \mathrm{m}$ height $\mathrm{b}$-Si structures. The physical property of b-Si structures was also important since the average width of the bSi nanostructures was very small $(280 \mathrm{~nm})$. Investigation of the photoresist coating on the b-Si nanostructures showed that the needle-like shaped nanostructures were able to retain their physical properties after the spin coating and the RTA cleaning processes. The optical properties of the b-Si solar cells were measured by using a photoluminescent (PL) measurement system. Based on the PL intensity, solar cells with the b-Si structures etched for 45 minutes appear to have the highest PL intensities than those etched for other lengths of time. High PL measurements indicated a low surface recombination, thus, b-Si solar cells with a 45 minutes etching time had the highest cell efficiency.

This thesis also reported the demonstration of the theoretical design and the experiment measurements of horizontally-grown silicon nanowires (SiNWs) structures on the surface of the c-Si solar cells. SiNW structures discussed in this thesis are in-plane self-assembled SiNWs, which were fabricated by a vapor-liquid-solid (VLS) mechanism process in a molecular beam epitaxy (MBE) chamber. The fabrication process of the SiNWs solar cell was also explained in Chapter 4. Based on the SiNWs growth process, 
the time of growth is the most important parameter, which directly affects the cell efficiency. Since the length of the SiNWs structures depended on the growth time, increasing the growth time increased the length and the surface coverage of SiNW structures. Based on the experimental results, SiNWs solar cells with a longer growth time had a better efficiency enhancement than those with a shorter growth times. The time of SiNW growth had a maximum of 4 hours since more growth time would affect the formation of independent SiNWs structures. Again, the applied SiNWs on c-Si solar cells were able to enhance the cell efficiency by reducing the surface reflectance. The optimized efficiency enhancement occurred when the SiNWs had 4-hours of growth time.

\subsection{Future work}

We demonstrated in this thesis the performance enhancement using cubic Ag MNPs as plasmonic scatters for light trapping in the c-Si solar cells. The MNPs were then optimized in terms of the shape, size, and dielectrical environment, and their effect on cell efficiency enhancement was characterized. However, due to the limitation of MNP fabrication technology and the requirement from LSPR, only two metals, gold $(\mathrm{Au})$ and silver (Ag), were considered as the MNP materials for the c-Si solar cell applications for the numerical simulations and experiment fabrication. Due to their stronger light scattering ability and lower fabrication cost, Ag MNPs were demonstrated to be a viable option for cell efficiency enhancement. Further investigation of MNPs will be focused on the use of new materials for MNPs, for example, aluminum (Al) MNPs. Interestingly, Al MNPs had an SPR peak that appeared in the UV regions, which allows detrimental parasitic absorption to be shifted outside of the solar spectrum peak range of the 
amorphous $\mathrm{Si}(\mathrm{a}-\mathrm{Si})$ solar cells [15]. Blue-shifting the plasmonic resonance would ensure enhancement rather than suppress the photocurrent response occurring over the entire solar spectrum at energies above the band-gap for a-Si, $1.74 \mathrm{eV}$. Further simulations must be conducted first to examine the effect of Al MNPs on bulk c-Si cells, as their thicknesses are much larger than the a-Si solar cell.

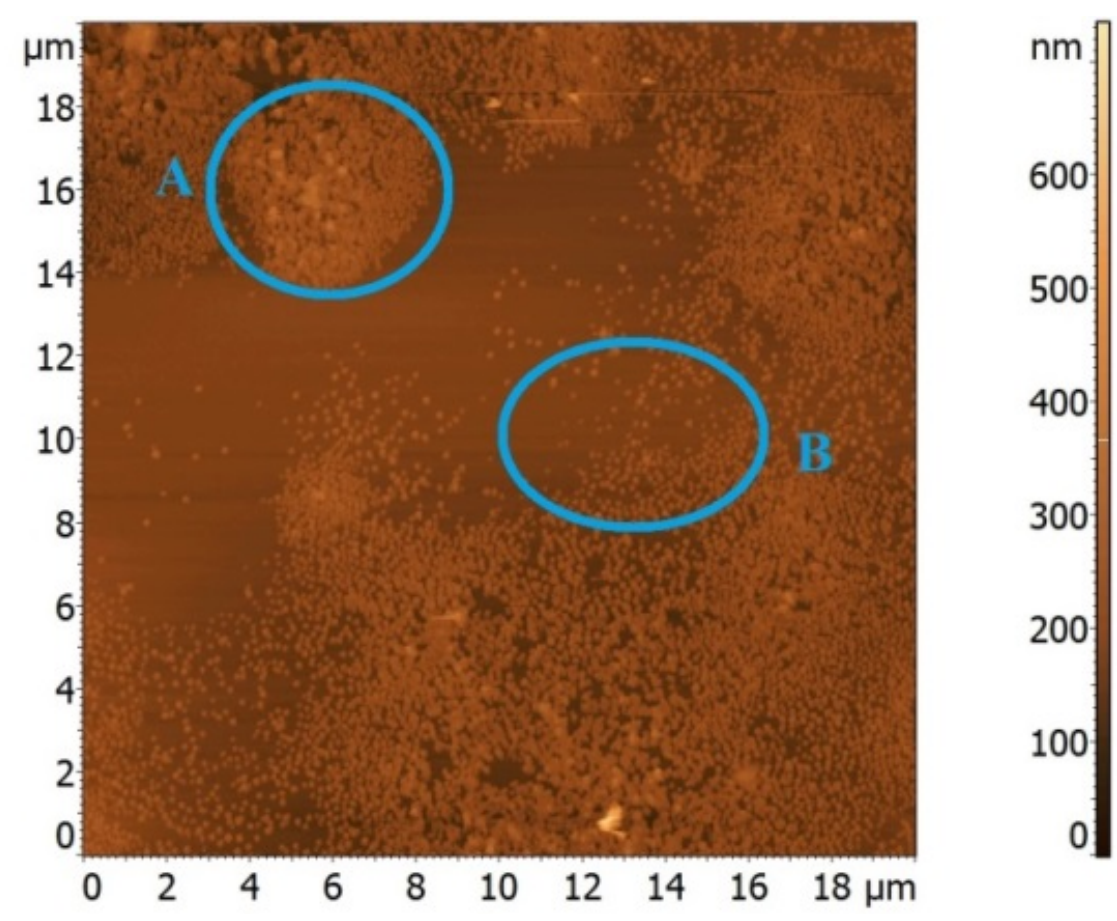

Figure 6-1: SEM topography image of cubic Ag MNPs deposited on c-Si solar cell

Fig. 6-1 shows an SEM topography image of a $20 \mu m \times 20 \mu m$ area c-Si solar cell front surface with the cubic Ag MNPs deposited by the LB deposition method. Due to the limitation of the fabrication tool and the deposition method, the cubic Ag MNPs were stacking together during the MNPs deposition process. They created an area that had a large amount of MNPs. Because the cubic Ag MNPs were stacking together, the shading loss in this area was much higher than it was on other areas. Increasing the shading loss of the solar cell decreases the cell efficiency. The deposition defects caused by the 
deposition method could be one of many reasons for the measured lower cell efficiency enhancement. In addition, the MNPs deposited on the solar cell surface may not have sufficient contact area from the solar cell to maintain their position via the deposition and cleaning processes. One possible method to redistribute MNPs on the solar cell is to apply the electrical beam (e-beam) lithography method, which is much more expensive than the LB deposition method but ensures the uniform distribution of the MNPs on the solar cells [15]. Another possible way to control the MNPs distribution is to use a preetching method on the solar cell before the MNP deposition. The surface textured structure etched by a pre-etching process could be used to guide the MNP positions on the solar cell, providing extra contact areas to the MNPs to avoid random movements during deposition. Further experimental verification on the deposition method should be carried out, and a high accuracy nanoparticle deposition method could also be developed to form a more uniform nanoparticle monolayer on large sized solar cells.

We demonstrated in the second part of this thesis the optical properties of the needleliked shape b-Si nanostructures by reducing the surface reflectivity of the solar cells. Due to the technique issues related to the ECR etching system since May 2014, we were unable to fabricate additional b-Si solar cells for experiments. Future fabrication must be done to complete the b-Si solar cell fabrication process, which will allow direct measurement of electrical properties of the b-Si solar cells. In order to demonstrate the cell efficiency enhancement from the b-Si nanostructures, experiment results of the b-Si solar cells would be important and useful for enhancing both optical and electrical properties. A continuation of the experimental work should be carried out with a defect removal process to reduce the huge surface recombination effects from the increased 
surface area. Besides the damage removal etching method, a rapid thermal annealing process could also be a key factor that could eliminate the defects in the b-Si structures made from the ECR etching process [64]. Continuing to optimize the simulation parameters and the fabrication process of the c-Si solar cells in terms of using plasmonic nanoparticles and black silicon nanostructures could well lead to a commercially feasible process for more efficient c-Si solar cells. 


\section{References}

[1] R. Production, P. Refi, and P. Trade, "BP Statistical Review of World Energy About this review Contents," no. June, 2013.

[2] M. Brower, D. Green, R. Hinrichs-rahlwes, S. Sawyer, M. Sander, R. Taylor, I. Giner-reichl, S. Teske, H. Lehmann, M. Alers, and D. Hales, "RENEWABLES 2014 GLOBAL STATUS REPORT,” 2014.

[3] A. Jäger-Waldau, "JRC Scientific and Policy Report: PV Status Report 2013," 2013.

[4] E. Becquerel, "Mémoire sur les effets électriques produits sous l'influence des rayons solaires," Comptes rendus l'Académie des Sci., vol. 9, pp. 561-567, 1839.

[5] C. Fritts, "On a New Form of Selenium Photocell," Am. J. Sci., vol. 26, p. 465, 1883.

[6] Jan Czochralski, "A new method for the measurement of the crystallization rate of metals," Zeitschrift für Phys. Chemie, vol. 92, pp. 219-221, 1918.

[7] D. M. Chapin, C. S. Fuller, and G. L. Pearson, "A New Silicon p-n Junction Photocell for Converting Solar Radiation into Electrical Power," J. Appl. Phys., vol. 25, no. 5, p. 676, 1954.

[8] N. P. D. Solarbuzz, "Solar PV Market Outlook,” no. October, pp. 1-16, 2013.

[9] Martin A. Green and Mark J. Keevers, "Optical properties of intrinsic silicon at 300 K,” Prog. Photovoltaics Res. Appl., vol. 3, no. 3, pp. 189-192, 1995.

[10] H. A. Atwater and A. Polman, "Plasmonics for improved photovoltaic devices.," Nat. Mater., vol. 9, no. 3, pp. 205-13, Mar. 2010.

[11] S. Pillai, K. R. Catchpole, T. Trupke, and M. a. Green, "Surface plasmon enhanced silicon solar cells," J. Appl. Phys., vol. 101, no. 9, p. 093105, 2007.

[12] K. R. Catchpole and A. Polman, "Design principles for particle plasmon enhanced solar cells," Appl. Phys. Lett., vol. 93, no. 19, p. 191113, Nov. 2008.

[13] A. Bottomley, D. Prezgot, and A. Ianoul, "Plasmonic properties of silver nanocube monolayers on high refractive index substrates," Appl. Phys. A, vol. 109, no. 4, pp. 869-872, Oct. 2012.

[14] M. a Ordal, L. L. Long, R. J. Bell, S. E. Bell, R. R. Bell, R. W. Alexander, and C. a Ward, "Optical properties of the metals $\mathrm{Al}, \mathrm{Co}, \mathrm{Cu}, \mathrm{Au}, \mathrm{Fe}, \mathrm{Pb}, \mathrm{Ni}, \mathrm{Pd}, \mathrm{Pt}, \mathrm{Ag}, \mathrm{Ti}$, 
and W in the infrared and far infrared.," Appl. Opt., vol. 22, no. 7, pp. 1099-1020, 1983.

[15] R. J. Veenkamp and W. N. Ye, "Plasmonic metal nanocubes for broadband light absorption enhancement in thin-film a-Si solar cells," J. Appl. Phys., vol. 115, no. 12, p. 124317 , Mar. 2014.

[16] H. a Atwater and A. Polman, "Plasmonics for improved photovoltaic devices.," Nat. Mater., vol. 9, no. 3, pp. 205-13, Mar. 2010.

[17] J. Bingham, "Fundamental and Applied Localized Surface Plasmon Resonance Spectroscopy Studies from Nanoparticle Arrays to Single Nanoparticles," Northwestern University, 2010.

[18] Q. Fu and W. Sun, "Mie theory for light scattering by a spherical particle in an absorbing medium," Appl. Opt., vol. 40, no. 9, pp. 1354-1361, 2001.

[19] J. Homola, S. S. Yee, and G. Gauglitz, "Surface plasmon resonance sensors: review,"Sensors Actuators, B Chem., vol. 54, pp. 3-15, 1999.

[20] "Chapter 3 Mie Theory.pdf." .

[21] M. A. Green, Solar cells: operating principles, technology, and system applications. Englewood Cliffs, NJ: Prentice-Hall, 1982.

[22] Richard C. Jaeger, "Introduction to microelectronic fabrication," Microelectron. Reliab., vol. 28, no. 5, p. 823, Jan. 1988.

[23] H. Jansen and M. De Boer, "The black silicon method: a universal method for determining the parameter setting of a fluorine-based reactive ion etcher in deep silicon trench etching with profile," J. ..., vol. 115, 1995.

[24] R. Di Mundo, M. Troia, F. Palumbo, M. Trotta, and R. d'Agostino, "Nanotexturing of Transparent Polymers with Plasma Etching: Tailoring Topography for a Low Reflectivity," Plasma Process. Polym., vol. 9, no. 10, pp. 947-954, Oct. 2012.

[25] Y. Xia, B. Liu, S. Zhong, and C. Li, "X-ray photoelectron spectroscopic studies of black silicon for solar cell," J. Electron Spectros. Relat. Phenomena, vol. 184, no. 11-12, pp. 589-592, Jan. 2012.

[26] J. Zhao, A. Wang, M. a. Green, and F. Ferrazza, “19.8\% Efficient 'Honeycomb' Textured Multicrystalline and 24.4\% Monocrystalline Silicon Solar Cells," Appl. Phys. Lett., vol. 73, no. 14, p. 1991, 1998. 
[27] R. ×. Einhaus, E. Vazsonyi, F. Duerinckx, J. Horzel, E. Van Kerschaver, J. Szlufcik, J. Nijs, and R. \# Mertens, "Recent progress with acidic texturing solutions on different multicrystalline silicon materials including ribbons," in 2nd World Conference and Exhibition on Photovoltaic Solar Energy Conversion, 1998, pp. 1630-1633.

[28] Y. Inomata, K. Fukui, and K. Shirasawa, "Surface texturing of large area multicrystalline silicon solar cells using reactive ion etching method," Sol. energy Mater. Sol. cells, vol. 48, pp. 237-242, 1997.

[29] C.-H. Hsu, J.-R. Wu, Y.-T. Lu, D. J. Flood, A. R. Barron, and L.-C. Chen, "Fabrication and characteristics of black silicon for solar cell applications: An overview," Mater. Sci. Semicond. Process., vol. 25, pp. 2-17, Sep. 2014.

[30] J. Yoo, "Reactive ion etching (RIE) technique for application in crystalline silicon solar cells," Sol. Energy, vol. 84, no. 4, pp. 730-734, Apr. 2010.

[31] K. Suzuki and S. Okudaira, "Microwave plasma etching," Japanese J. ..., vol. 34, pp. 953-957, 1977.

[32] G. Kumaravelu, M. . Alkaisi, a Bittar, D. Macdonald, and J. Zhao, "Damage studies in dry etched textured silicon surfaces," Curr. Appl. Phys., vol. 4, no. 2-4, pp. 108-110, Apr. 2004.

[33] K. Lee, M.-H. Ha, J. H. Kim, and J.-W. Jeong, "Damage-free reactive ion etch for high-efficiency large-area multi-crystalline silicon solar cells," Sol. Energy Mater. Sol. Cells, vol. 95, no. 1, pp. 66-68, Jan. 2011.

[34] J. Yoo, G. Yu, and J. Yi, "Black surface structures for crystalline silicon solar cells," Mater. Sci. Eng. B, vol. 159-160, pp. 333-337, Mar. 2009.

[35] G. Xiao, B. Liu, J. Liu, and Z. Xu, "The study of defect removal etching of black silicon for solar cells," Mater. Sci. Semicond. Process., vol. 22, pp. 64-68, Jun. 2014.

[36] L. Yu, M. Xu, J. Xu, Z. Xue, Z. Fan, G. Picardi, F. Fortuna, J. Wang, J. Xu, Y. Shi, K. Chen, and P. Roca i Cabarrocas, "In-Plane Epitaxial Growth of Silicon Nanowires and Junction Formation on Si(100) Substrates," Nano Lett., vol. 14, no. 100, pp. 6469-6474, 2014.

[37] C. S. Cojocaru, J. M. Padovani, T. Wade, C. Mandoli, G. Jaskierowicz, J. E. Wegrowe, A. Fontcuberta i Morral, and D. Pribat, "Conformal anodic oxidation of aluminum thin films," Nano Lett., vol. 5, pp. 675-680, 2005.

[38] R. S. Wagner and W. C. Ellis, "Vapor-liquid-solid mechanism of single crystal growth," Appl. Phys. Lett., vol. 4, no. 1964, pp. 89-90, 1964. 
[39] E. I. Givargizov, Highly anisotropic crystals, 1st ed. Springer Netherlands, 1987.

[40] V. Schmidt, J. V. Wittemann, S. Senz, and U. Gósele, "Silicon nanowires: A review on aspects of their growth and their electrical properties," Adv. Mater., vol. 21, pp. 2681-2702, 2009.

[41] J. Weyher, "Some notes on the growth kinetics and morphology of VLS silicon crystals grown with platinum and gold as liquid-forming agents," J. Cryst. Growth, vol. 43, pp. 235-244, 1978.

[42] D. D. D. Ma, C. S. Lee, F. C. K. Au, S. Y. Tong, and S. T. Lee, "Small-diameter silicon nanowire surfaces.," Science, vol. 299, no. March, pp. 1874-1877, 2003.

[43] Y. Wu, Y. Cui, L. Huynh, C. J. Barrelet, D. C. Bell, and C. M. Lieber, "Controlled growth and structures of molecular-scale silicon nanowires," Nano Lett., vol. 4, no. Figure 1, pp. 433-436, 2004.

[44] M. S. Islam, S. Sharma, T. I. Kamins, and R. S. Williams, "Ultrahigh-density silicon nanobridges formed between two vertical silicon surfaces," Nanotechnology, vol. 15, pp. L5-L8, 2004.

[45] R. He, D. Gao, R. Fan, A. I. Hochbaum, C. Carraro, R. Maboudian, and P. Yang, "Si nanowire bridges in microtrenches: Integration of growth into device fabrication," Adv. Mater., vol. 17, pp. 2098-2102, 2005.

[46] Y. a Akimov, W. S. Koh, and K. Ostrikov, "Enhancement of optical absorption in thin-film solar cells through the excitation of higher-order nanoparticle plasmon modes.," Opt. Express, vol. 17, no. 12, pp. 10195-10205, 2009.

[47] F. J. Beck, a. Polman, and K. R. Catchpole, "Tunable light trapping for solar cells using localized surface plasmons," J. Appl. Phys., vol. 105, no. 11, p. 114310, 2009.

[48] J. S. Ã, H. Lee, K. Cho, E. Lee, and J. Kim, "Industrial 6 Inch Multicrystalline Silicon Solar Cells Fabricated Using Reactive Ion Etching with Efficiency Exceeding $18 \%$ Industrial 6 Inch Multicrystalline Silicon Solar Cells Fabricated Using Reactive Ion Etching with Efficiency Exceeding 18 \%," vol. 14.

[49] S. Pillai, F. J. Beck, K. R. Catchpole, Z. Ouyang, and M. a. Green, "The effect of dielectric spacer thickness on surface plasmon enhanced solar cells for front and rear side depositions," J. Appl. Phys., vol. 109, no. 2011, 2011.

[50] J. Oh, H.-C. Yuan, and H. M. Branz, "An 18.2\%-efficient black-silicon solar cell achieved through control of carrier recombination in nanostructures.," Nat. Nanotechnol., vol. 7, no. 11, pp. 743-8, Nov. 2012. 
[51] M. D. Abbott, J. E. Cotter, F. W. Chen, T. Trupke, R. a. Bardos, and K. C. Fisher, "Application of photoluminescence characterization to the development and manufacturing of high-efficiency silicon solar cells," J. Appl. Phys., vol. 100, no. 11 , p. 114514, 2006.

[52] S. Kalem, P. Werner, and Ö. Arthursson, "Black silicon with high density and high aspect ratio nanowhiskers," ..., pp. 1-22, 2011.

[53] T. Trupke, R. a. Bardos, M. C. Schubert, and W. Warta, "Photoluminescence imaging of silicon wafers," Appl. Phys. Lett., vol. 89, no. 4, p. 044107, 2006.

[54] T. Trupke and W. Mcmillan, "Photoluminescence imaging speeds solar cell inspection," Laser Focus World, vol. 46, no. 12, p. 35, 2010.

[55] D. Baek, S. Rouvimov, B. Kim, T.-C. Jo, and D. K. Schroder, "Surface recombination velocity of silicon wafers by photoluminescence," Appl. Phys. Lett., vol. 86, no. 11, p. 112110, 2005.

[56] S. N. Dedyulin, G. Fanchini, and L. V. Goncharova, "Reticular Growth of Silicon Ridges: Random Walk in Two Dimensions," Cryst. Growth Des., vol. 14, pp. 1193-1198, 2014.

[57] S. E. Skrabalak, L. Au, X. Li, and Y. Xia, "Facile synthesis of Ag nanocubes and Au nanocages.," Nat. Protoc., vol. 2, no. 9, pp. 2182-90, Jan. 2007.

[58] N. Ahamad and A. Ianoul, "Using phospholipids to control interparticle distance in SERS-active substrates," J. Phys. Chem. C, pp. 3587-3594, 2011.

[59] B. J. Wiley, S. H. Im, Z.-Y. Li, J. McLellan, A. Siekkinen, and Y. Xia, "Maneuvering the surface plasmon resonance of silver nanostructures through shape-controlled synthesis.," J. Phys. Chem. B, vol. 110, no. 32, pp. 15666-75, Aug. 2006.

[60] A. Bottomley, D. Prezgot, and A. Ianoul, "Plasmonic properties of silver nanocube monolayers on high refractive index substrates," Appl. Phys. A, vol. 109, no. 4, pp. 869-872, Oct. 2012.

[61] J. Zuloaga, E. Prodan, and P. Nordlander, "Quantum description of the plasmon resonances of a nanoparticle dimer.," Nano Lett., vol. 9, no. 2, pp. 887-91, Feb. 2009.

[62] S. N. Dedyulin and L. V. Goncharova, "Thermodynamic and kinetic control of the lateral Si wire growth," Appl. Phys. Lett., vol. 104, pp. 12-16, 2014. 
[63] T. Konishi, T. Yao, M. Tajima, H. Ohshima, H. Ito, and T. Hattori, "Characterization of HF-Treated Si Surfaces by Photoluminescence Spectroscopy," Jpn. J. Appl. Phys., vol. 31, no. Part 2, No. 9A, pp. L1216-L1218, Sep. 1992.

[64] Y. Su, S. Li, Z. Wu, Y. Yang, Y. Jiang, J. Jiang, Z. Xiao, P. Zhang, and T. Zhang, "High responsivity MSM black silicon photodetector," Mater. Sci. Semicond. Process., vol. 16, no. 3, pp. 619-624, Jun. 2013.

[65] P. Doshi, A. Rohatgi, M. Ropp, Z. Chen, D. Ruby, and D. L. Meier, "Rapid thermal processing of high-efficiency silicon solar cells with controlled in-situ annealing," Proc. 1994 IEEE 1st World Conf. Photovolt. Energy Convers. WCPEC (A Jt. Conf. PVSC, PVSEC PSEC), vol. 2, pp. 1299-1302, 1994.

[66] F. Reurings, C. Rauch, F. Tuomisto, R. E. Jones, K. M. Yu, W. Walukiewicz, and W. J. Schaff, "Defect redistribution in postirradiation rapid-thermal-annealed InN," Phys. Rev. B - Condens. Matter Mater. Phys., vol. 82, no. October, pp. 1-4, 2010. 


\section{Appendix}

\section{Appendix A : The Mie Theory}

We can determine the net rate of change of energy as light through the spherical particle by applying the complex Poynting vector law. Afterward, we can use the relative equations between the light and energy to obtain the absorption, scattered and extinction cross sections ${ }^{[7]}$.

$$
\begin{gathered}
C_{\text {scat }}=\frac{W_{a b s}}{I_{\text {inc }}} \\
C_{\text {scat }}=\frac{W_{\text {scat }}}{I_{\text {inc }}} \\
C_{\text {ext }}=\frac{W_{\text {ext }}}{I_{\text {inc }}}
\end{gathered}
$$

Where $W_{a b s}, W_{\text {scat }}$ and $W_{\text {ext }}$ are the absorption, scattered and extinction energies given respectively by their functions. The variable $I_{i n c}$ represents the incident light intensity on the surface of the spherical particle. By applying the boundary conditions of the electromagnetic fields across the particle surface $r=a$, we can determine following equation at the surface of the particle ${ }^{[8]}$.

$$
\left(E \times H^{*}\right) \cdot r^{2}=\left[\left(E_{\text {inc }}+E_{\text {scat }}\right) \times\left(H_{\text {inc }}+H_{\text {scat }}\right)^{*}\right] \cdot r^{2}
$$

Thus

$$
\left(E \times H^{*}\right) \cdot r^{2}=\left(E_{\text {int }}+H_{\text {int }}^{*}\right) \cdot r^{2}
$$

We can obtain the absorption energy function by applying the absorption field function. The absorption energy $W_{a b s}$ can be expressed as equation below ${ }^{[8]}$.

$$
\begin{gathered}
W_{a b s}=\frac{1}{2} \operatorname{Re} \int_{0}^{2 \pi} \int_{0}^{\pi}\left(E_{t} \times H_{t}^{*}\right) r^{2} \sin \theta d \theta d \phi \\
W_{a b s}=\frac{1}{2} \operatorname{Re} \int_{0}^{2 \pi} \int_{0}^{\pi}\left(E_{i n t, \theta} \times H_{i n t, \phi}^{*}-E_{i n t, \phi} \times H_{i n t, \theta}^{*}\right) r^{2} \sin \theta d \theta d \phi
\end{gathered}
$$

Therefore, we can obtain the energy functions for the scattered and extinction energies by applying the scattered and internal field functions ${ }^{[7]}$.

$$
W_{\text {scat }}=\frac{1}{2} \operatorname{Re} \int_{0}^{2 \pi} \int_{0}^{\pi}\left(E_{\text {scat }} \times H_{\text {scat }}^{*}\right) r^{2} \sin \theta d \theta d \phi
$$




$$
\begin{gathered}
W_{\text {scat }}=\frac{1}{2} \operatorname{Re} \int_{0}^{2 \pi} \int_{0}^{\pi}\left(E_{\text {scat }, \theta} \times H_{\text {scat }, \phi}^{*}-E_{\text {scat }, \phi} \times H_{\text {scat }, \theta}^{*}\right) r^{2} \sin \theta d \theta d \phi \\
W_{\text {scat }}=\frac{1}{2} \operatorname{Re} \int_{0}^{2 \pi} \int_{0}^{\pi}\left(E_{\text {inc }} \times H_{\text {scat }}^{*}\right) r^{2} \sin \theta d \theta d \phi \\
W_{\text {ext }}=\frac{1}{2} \operatorname{Re} \int_{0}^{2 \pi} \int_{0}^{\pi}\left(E_{\text {inc }, \phi} \times H_{\text {scat }, \theta}^{*}-E_{\text {inc }, \theta} \times H_{\text {scat }, \phi}^{*}-E_{\text {scat }, \theta} \times H_{\text {inc }, \phi}^{*}+E_{\text {scat }, \phi}\right. \\
\left.\times H_{\text {inc }, \phi}^{*}\right) r^{2} \sin \theta d \theta d \phi
\end{gathered}
$$

Note that the absorbed energy for spherical particle can be related to the scatted and extinction energies by equation below ${ }^{[7]}$.

$$
W_{a b s}=W_{e x t}-W_{\text {scat }}
$$

Thus, the equations for the scattered and extinction cross sections can re-express to the equations below.

$$
\begin{gathered}
C_{s c a t}=\frac{2 \pi}{k_{m}^{2}} \sum_{l=1}^{\infty}(2 l+1)\left(a_{l}\left|H_{l, m}\right|^{2}+b_{l}\left|F_{l, m}\right|^{2}\right) \\
C_{e x t}=\frac{2 \pi}{k_{m}^{2}} \operatorname{Re} \sum_{l=1}^{\infty}(2 l+1)\left(a_{l}\left|H_{l, m}\right|^{2}+b_{l}\left|F_{l, m}\right|^{2}\right)
\end{gathered}
$$

Where $H_{l, m}$ and $F_{l, m}$ are angular functions which will relate to the $\theta$-angular functions $\Pi_{l, m}$ and $\mathrm{T}_{l, m}$ by equations below.

$$
\begin{aligned}
& H_{l, m}=\frac{2}{l(l+1)} \sum_{m=-1}^{l} \frac{(l-m) !}{(l+m) !}\left|T_{l, m}\right|^{2} \\
& F_{l, m}=\frac{2}{l(l+1)} \sum_{m=-1}^{l} \frac{(l-m) !}{(l+m) !}\left|\Pi_{l, m}\right|^{2}
\end{aligned}
$$

For a plane wave, the incident angle $\theta=0$ and we assume all terms in system will vanish except $l$ and $m$.

The number of $m$ will set to 1 . The scattered and extinction cross sections in electromagnetic fields can simplify to equations below ${ }^{[6]}$.

$$
\begin{gathered}
C_{\text {scat }}=\frac{2 \pi}{k_{m}^{2}} \sum_{l=1}^{\infty}(2 l+1)\left(\left|a_{l}\right|^{2}+\left|b_{l}\right|^{2}\right) \\
C_{\text {ext }}=\frac{2 \pi}{k_{m}^{2}} R e \sum_{l=1}^{\infty}(2 l+1)\left(a_{l}+b_{l}\right)
\end{gathered}
$$




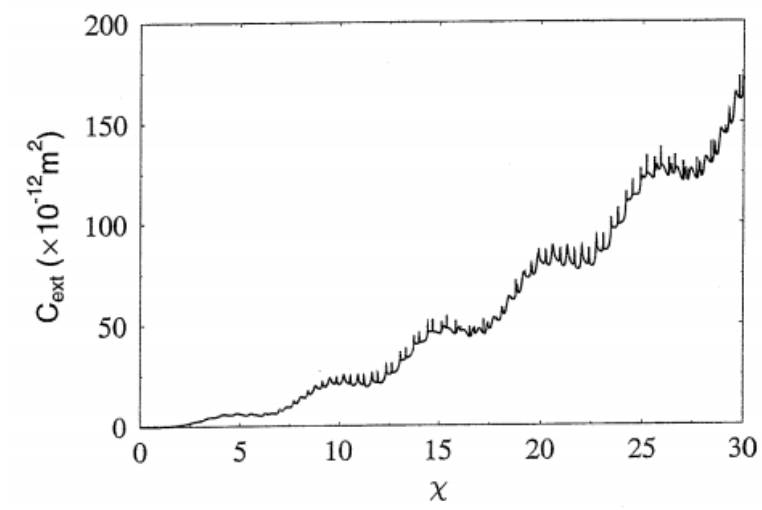

Figure 4: Extinction cross section for a dielectric spherical particle with an index of $N_{S}=1.59$ in air, $\lambda=1 u^{6}$

Note that the efficiency factor or Q-factor of the spherical particle is a dimensionless parameter which is associated with cross section of the spherical particle. The extinction and scattering efficiencies for the spherical particle surrounding with nonabsorbing medium related to cross sections can be expressed as equations below ${ }^{[7]}$.

$$
\begin{aligned}
Q_{\text {scat,nonabs }} & =\frac{C_{\text {scat }}}{G} \\
Q_{\text {ext.nonabs }} & =\frac{C_{\text {ext }}}{G}
\end{aligned}
$$

The $\mathrm{G}$ parameter in equation is defined as the geometrical cross section, which is $G=\pi a^{2}$ for a spherical particle and $\alpha$ is the spherical particle radius.

When the surrounding medium is absorbing, the scattering and extinction efficiency from the Mie theory need to be modified for light scattering by particle to be described. Therefore, the rate of energy incident on the spherical particle in an absorbing medium has to participate in efficiency equations. The expression of the rate of energy incident on the particle is equation below ${ }^{[9]}$.

$$
f=\frac{2 \pi a^{2}}{\eta^{2}} I_{0}\left[1+(\eta-1) e^{\eta}\right]
$$

Where

$$
\eta=\frac{4 \pi a m_{i}}{\lambda_{0}}, I_{0}=\left[\frac{m_{r}}{2 c \mu}\right]\left|E_{0}\right|^{2}
$$

In the equation of the rate of incident energy, $c$ is the speed of light in vacuum, and $m_{i}$ and $m_{r}$ are the imaginary and real parts of the complex refractive index of the surrounding medium. The absorption, 
extinction and scattering efficiencies for a spherical particle embedded in absorbing medium can be expressed as equations below ${ }^{[8]}$.

$$
\begin{gathered}
Q_{a b s}=\frac{W_{a b s}}{f} \\
Q_{e x t}=\frac{W_{e x t}}{f} \\
Q_{\text {scat }}=\frac{W_{\text {scat }}}{f}
\end{gathered}
$$

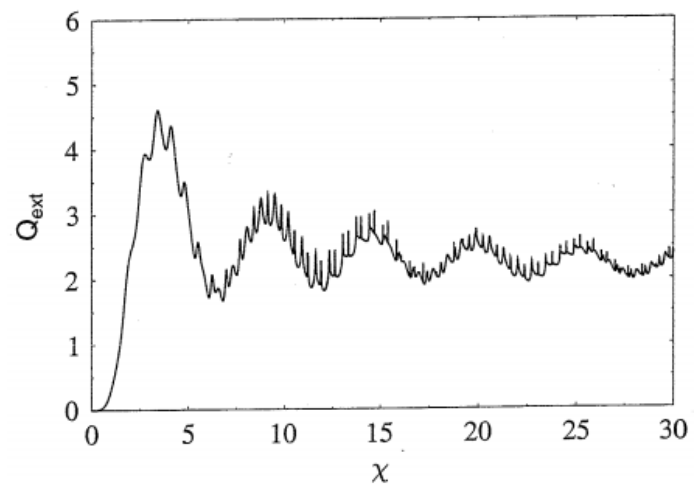

Figure 5: Extinction efficiency for a dielectric spherical particle with an index of $N_{s}=1.59$ in air, $\lambda=1 \mathrm{um}^{[5]}$ 


\section{Appendix B: Fabrication Process Runsheet for c-Si solar cells}

STARTING MATERIAL:

5 - 1-4 $\Omega \mathrm{cm} p<100>$ (pick lowest res. wafers) PV13-1,2,3,4,5

Record resistivity's . PV13-1 $=2$ ohm-cm, PV13-2 $=1.9$ ohm-cm,

PV13=2.16 ohm-cm, PV13-4 = 2.2 ohm-cm, PV13-5=2.2 ohm $-\mathrm{cm}$

Brief Outline

1. SCREEN OXIDATION - 400nm

2. DEVICE WELL P.E. - RIE/BOE etch

3. PHOSPHORUS DIFFUSIONS

- 875deg C (standard - PV2,3,5,6),

- 1000deg C (standard PV4)

4. BACK OXIDE REMOVAL - RIE

5. BACKSIDE 1\% HF SWAB TO HYDROPHOBIC

6. BACK METALLIZATION (0.5um Aluminum)

7. METAL SINTER 1-500 deg C N2

8. METAL LIFT-OFF LITHOGRAPHY

9. FRONT METALLIZATION (Titanium/Silver)

10. METAL LIFT-OFF

11. METAL SINTER $2-400 \mathrm{deg} \mathrm{H} 2$

12. ANTIREFLECTION COATING (spin on Titaniumsilica)

1. SCREEN OXIDATION

Apex recipe\# Apex Run\#

$400 \mathrm{~nm}$

a) RCA clean

b) Oxidation tube temperature $1000^{\circ} \mathrm{C}$

5 min preheat $\quad \mathrm{O}_{2}$

2 " every 6 sec push $\quad \mathrm{O}_{2}$

10 min oxidation $\quad$ dry $\mathrm{O}_{2}$

65min wet oxidation $\quad \mathrm{O}_{2}+\mathrm{H}_{2}$

20 min oxidation $\quad \mathrm{O}_{2}+2 \% \mathrm{HCl}$

20 min anneal $\quad \mathrm{N}_{2}$

2 " every $15 \mathrm{sec}$ pull $\quad \mathrm{N}_{2}$

10 min cool $\quad \mathrm{N}_{2}$

target thickness: $400 \mathrm{~nm}$

thickness measured on wafer:

\section{DEVICE WELL P.E.}

Mask CU-

Dark field

a) PlasmaPreen condition, (Plasmapreen condition not required if coming out of furnace tube) 
HMDS oven, apply S1811 photoresist, prebake, expose, develop, postbake, descum

(See Appendix A for standard photoresist procedure)

b) RIE etch: Fronts

etch $\sim 10 \mathrm{~min} @ \sim 40 \mathrm{~nm} / \mathrm{min}$ in 15\% O2 + 85\% $\mathrm{CHF}_{3}$

RF Power: - $350 \mathrm{~W}$ Pressure: $150 \mathrm{mTorr}$

Leave $\sim 70 \mathrm{~nm}$ of oxide in scribe channels

as measured with Nanospec.

c) etch in buffered HF @ $20^{\circ} \mathrm{C}$ until bare Si exposed

(scribe channels will go hydrophobic)

estimated etch time: 2.5 to $3 \mathrm{~min}$

actual etch times:

c) strip PR in PlasmaPreen

\section{PHOSPHORUS DIFFUSIONS}

Include one 4" $p$ type $<100>, 1-5 \Omega \mathrm{cm}$ test wafer in each diffusion

a) RCA clean

b) First diffusion. Include PV13-1 and one test wafer.

pre-deposition source: $\mathrm{POCl}_{3} @ 20^{\circ} \mathrm{C}$ tube temperature: $\mathbf{1 0 0 0}^{\circ} \mathrm{C}$

$$
\text { Apex recipe\#___ Apex Run\# }
$$

5 min preheat $\quad \mathrm{N}_{2}+\mathrm{O}_{2}$

$30 \mathrm{sec}$ push $\quad \mathrm{N}_{2}+\mathrm{O}_{2}$

5 min warm-up $\quad \mathrm{N}_{2}+\mathrm{O}_{2}$

5 min dope $\quad \mathrm{N}_{2}+\mathrm{O}_{2}+\mathrm{N}_{2} /$ dopant

$10 \mathrm{~min}$ flush/drive-in $\mathrm{N}_{2}+\mathrm{O}_{2}$

30 sec pull $\quad \mathrm{N}_{2}+\mathrm{O}_{2}$

10 min cool $\quad \mathrm{N}_{2}$

remove phosphosilicate glass: etch $40 \mathrm{sec}$ in $1 \% \mathrm{HF}$

measure sheet resistance test wafer

sheet resistance:

(expecting $\sim 9-10 \mathrm{ohm} /$ square on test wafer $\sim 2 \mathrm{mv}$ ) on test wafer. 
c) Second diffusion PV13-2, 3, 4, 6,7 and one 1-5 ohm-cm test wafer

Pre-deposition source: $\mathrm{POCl}_{3} @ 20^{\circ} \mathrm{C}$ tube temperature: $\mathbf{8 7 5}^{\circ} \mathrm{C}$

$$
\text { Apex recipe\#___ Apex Run\# }
$$

5 min preheat $\quad \mathrm{N}_{2}+\mathrm{O}_{2}$

$30 \mathrm{sec}$ push $\quad \mathrm{N}_{2}+\mathrm{O}_{2}$

5 min warm-up $\quad \mathrm{N}_{2}+\mathrm{O}_{2}$

5 min dope $\quad \mathrm{N}_{2}+\mathrm{O}_{2}+\mathrm{N}_{2} /$ dopant

$10 \mathrm{~min}$ flush/drive-in $\mathrm{N}_{2}+\mathrm{O}_{2}$

30 sec pull $\quad \mathrm{N}_{2}+\mathrm{O}_{2}$

10 min cool $\quad \mathrm{N}_{2}$

remove phosphosilicate glass: etch $40 \mathrm{sec}$ in $1 \% \mathrm{HF}$

measure sheet resistance test wafer

sheet resistance:

(expecting $\sim 59 \mathrm{ohm} / \mathrm{square}$, or $13 \mathrm{mv}$ on test wafer)

( 71ohm/square on PV wafers)

\section{BACK OXIDE REMOVAL}

a) Flood coat front of wafers with PR to protect.

b) Dry etch in MRC RIE approx. 15min, O2/CHF3, 350W RF, 150mtorr.

c) remove PR with Acetone/IPA/Plasmapreen

\section{Backside 1\% HF swab to hydrophobic}

\section{BACK METALLIZATION}

Al e-beam evaporation on backs target thickness: $0.5 \mu \mathrm{m}$ actual thickness: 
7. METAL SINTER 1 -- NOTE in N2 RUN One wafer through as test to make sure looks ok. Tube temperature $500 \mathrm{C}$

5 min preheat $\mathbf{N}_{2}$

5 min tube purge $\quad \mathrm{N}_{2}$

20 second push $\quad \mathrm{N}_{2}$

10 min anneal $\mathrm{N}_{2}$

$20 \mathrm{sec}$ pull

5 min cool

$\mathrm{N}_{2}$

$\mathrm{N}_{2}$

10 min tube purge $\quad \mathrm{N}_{2}$

\section{METAL LIFT-OFF LITHOGRAPHY Mask CU-_ Dark Field}

PlasmaPreen condition, HMDS oven

MicroChem LOR lift-off resist.

Flood coat and spin LOR 10B

Bake LOR layer on hotplate for 5 min @ 185-190 ${ }^{\circ} \mathrm{C}$

Flood coat and spin on S1811 positive PR@4000 RPM

Prebake on hot plate $@ 100{ }^{\circ} \mathrm{C}$ for $1 \mathrm{~min}$.

Align wafer to mask

Expose _ seconds. Use standard exposure time for S1811

Develop the Bi layer in MF-321 developer for 45 seconds

Inspect at 100x (minimum) brightfield and darkfield.

DO NOT POSTBAKE

9. FRONT METALLIZATION - Balzers evaporator.

a) Oxygen plasma descum, 1 min. Maximum @ $100 \mathrm{~W}, 300 \mathrm{mT}$

b) Flash 1\% HF to remove native oxide

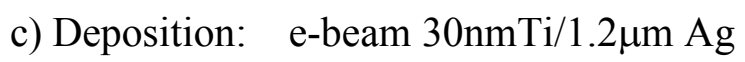

actual thickness:

\section{METAL LIFT-OFF}

Lift-off the layer in Microposit remover 1165 as follows: 
Soak wafer in remover at $80^{\circ} \mathrm{C}$

Use two separate beakers, combined time may be $90 \mathrm{~min}$.

Rinse gently in running DI water for $15 \mathrm{~min}$.

Inspect for complete lift-off

If required, return the wafer to the second beaker of 1165 solution.

to remove residue.

Rinse wafer, then dry

NOTE: silver may look brown after this step but should clear up after sinter step.

\section{METAL SINTER 2}

Tube temperature $400 \mathrm{C}$

5 min preheat $\mathrm{N}_{2}$

5 min tube purge

20 second push

$\mathrm{H}_{2}$

10 min anneal

$\mathrm{H}_{2}$

$20 \mathrm{sec}$ pull

$\mathrm{H}_{2}$

5 min cool

$\mathrm{H}_{2}$

10 min tube purge

$\mathrm{H}_{2}$

$\mathrm{N}_{2}$ 


\section{Appendix C : Fabrication Process Runsheet for b-Si solar cells}

\begin{tabular}{|c|c|c|c|c|c|c|c|c|c|c|c|c|c|}
\hline Date & Sample & $\begin{array}{l}\text { Etch } \\
\text { Time } \\
(\min )\end{array}$ & FWD & RFL & $\begin{array}{l}\text { RF } \\
\text { FWD }\end{array}$ & $\begin{array}{l}\text { RF } \\
\text { RFL }\end{array}$ & $\begin{array}{l}\mathrm{sccm} \\
\text { SF6 }\end{array}$ & $\begin{array}{l}\mathrm{sccm} \\
\mathrm{O} 2\end{array}$ & $\mathrm{He}$ & $\begin{array}{l}\text { Gas } \\
\text { Press } \\
\text { (mTorr) }\end{array}$ & $\begin{array}{l}\text { Base } \\
\text { Press } \\
\text { (mTorr) }\end{array}$ & Temp & Comments \\
\hline $\begin{array}{l}\text { May } 13 \\
2014\end{array}$ & $\# 1$ & 5 & 246 & 2 & 10 & 0 & 3.65 & 6.78 & 4.5 & 6 & $1 * 10-5$ & -63 & $\begin{array}{l}\text { only slightly } \\
\text { brown }\end{array}$ \\
\hline $\begin{array}{l}\text { May } 13 \\
2014\end{array}$ & $\# 1$ & 10 & 246 & 2 & 10 & 0 & 3.65 & 6.78 & 4.5 & 6 & $1 * 10-5$ & -40 & greyish black \\
\hline $\begin{array}{l}\text { May 14 } \\
2014\end{array}$ & $\# 1$ & 15 & 247 & 2 & 9 & 0 & 3.65 & 6.78 & 4.5 & 6 & $1 * 10-5$ & -59.5 & $\begin{array}{l}\text { looks black } \\
\text { after total } 35 \\
\text { min }\end{array}$ \\
\hline $\begin{array}{l}\text { May } 14 \\
2014\end{array}$ & $\# 2$ & 30 & 247 & 2 & 10 & 0 & 3.65 & 6.78 & 4.5 & 6 & $\begin{array}{l}1.3 * 10- \\
5\end{array}$ & -58.5 & $\begin{array}{l}\text { brown } \\
\text { grey(move on } \\
\text { the grey side) }\end{array}$ \\
\hline $\begin{array}{l}\text { May } 14 \\
2014\end{array}$ & $\# 3$ & 35 & 261 & 2 & 10 & 0 & 3.65 & 6.78 & 4.5 & 6 & $\begin{array}{l}5.3 * 10- \\
5\end{array}$ & -30.9 & $\begin{array}{l}\text { discontinued } \\
\text { after } 21 \\
\text { minutes } \\
\text { remaining } 17 \\
\text { minutes left to } \\
\text { the next day }\end{array}$ \\
\hline $\begin{array}{l}\text { May } 14 \\
2014\end{array}$ & $\# 3$ & 17 & 255 & 2 & 9 & 0 & 3.65 & 6.78 & 4.5 & 6 & $2 * 10-5$ & -48.9 & $\begin{array}{l}\text { looks very } \\
\text { black under } \\
\text { light }\end{array}$ \\
\hline $\begin{array}{l}\text { May } 15 \\
2014\end{array}$ & $\# 4$ & 35 & 247 & 2 & 10 & 0 & 3.65 & 6.78 & 4.5 & 6 & $\begin{array}{l}7.4 * 10- \\
6\end{array}$ & -39 & $\begin{array}{l}\text { look greyish } \\
\text { black }\end{array}$ \\
\hline $\begin{array}{l}\text { May 15 } \\
2014\end{array}$ & $\# 5$ & 40 & 256 & 2 & 10 & 0 & 3.65 & 6.78 & 4.5 & 6 & $\begin{array}{l}5.1 * 10- \\
6\end{array}$ & -35.3 & $\begin{array}{l}\text { black, during } \\
\text { etching } \\
\text { pretention } \\
\text { changed }\end{array}$ \\
\hline $\begin{array}{l}\text { May 20 } \\
2014\end{array}$ & $\# 6$ & 45 & 257 & 2 & 10 & 0 & 3.65 & 6.78 & 4.5 & 6 & $\begin{array}{l}2.5 * 10- \\
6\end{array}$ & -57.1 & $\begin{array}{l}\text { black with a } \\
\text { slight begin } \\
\text { brown }\end{array}$ \\
\hline $\begin{array}{l}\text { May } 20 \\
2014\end{array}$ & $\# 7$ & 50 & 244 & 2 & 10 & 0 & 3.65 & 6.78 & 4.5 & 6 & $\begin{array}{l}1.5 * 10- \\
6\end{array}$ & -42.5 & $\begin{array}{l}\text { completely } \\
\text { grey }\end{array}$ \\
\hline $\begin{array}{l}\text { May 20 } \\
2014\end{array}$ & $\# 8$ & 20 & 300 & 2 & 10 & 0 & 7.3 & 6.78 & 4.5 & 6 & $\begin{array}{l}5.9 * 10- \\
6\end{array}$ & -46.5 & $\begin{array}{l}\text { no change in } \\
\text { the sample }\end{array}$ \\
\hline $\begin{array}{l}\text { May 20 } \\
2014\end{array}$ & $\# 8$ & 35 & 299 & 2 & 10 & 0 & 7.3 & 6.78 & 4.5 & 6 & $\begin{array}{l}5.8 * 10- \\
6\end{array}$ & -40.1 & $\begin{array}{l}\text { almost no } \\
\text { change on the } \\
\text { sample }\end{array}$ \\
\hline $\begin{array}{l}\text { May 20 } \\
2014\end{array}$ & $\# 9$ & 50 & 246 & 2 & 10 & 0 & 3.65 & 6.78 & 4.5 & 6 & $\begin{array}{l}1.5 * 10- \\
6\end{array}$ & -41.7 & $\begin{array}{l}\text { sliver black } \\
\text { (more on the } \\
\text { grey side), } \\
\text { damage after } \\
\text { etching }\end{array}$ \\
\hline $\begin{array}{l}\text { May } 21 \\
2014\end{array}$ & $\# 10$ & 45 & 266 & 2 & 10 & 0 & 3.65 & 6.78 & 4.5 & 6 & $\begin{array}{l}6.4 * 10- \\
6\end{array}$ & -57.5 & $\begin{array}{l}\text { black with a } \\
\text { little brown }\end{array}$ \\
\hline $\begin{array}{l}\text { May } 21 \\
2014\end{array}$ & $\# 11$ & 40 & 254 & 2 & 10 & 0 & 3.65 & 6.78 & 4.5 & 6 & $\begin{array}{l}1.4 * 10- \\
5\end{array}$ & -41.7 & grey black \\
\hline $\begin{array}{l}\text { May } 21 \\
2014\end{array}$ & $\# 10$ & 45 & 263 & 2 & 10 & 0 & 3.65 & 6.78 & 4.5 & 6 & $5 * 10-6$ & -42.1 & $\begin{array}{l}\text { original } \\
\text { sample \#10 }\end{array}$ \\
\hline
\end{tabular}




\begin{tabular}{|c|c|c|c|c|c|c|c|c|c|c|c|c|c|}
\hline & & & & & & & & & & & & & $\begin{array}{l}\text { was broken, } \\
\text { looks black } \\
\text { with little } \\
\text { brown }\end{array}$ \\
\hline $\begin{array}{l}\text { May } 22 \\
2014\end{array}$ & $\# 12$ & 45 & 261 & 2 & 10 & 0 & 3.65 & 6.78 & 4.5 & 6 & $\begin{array}{l}8.8 * 10- \\
6\end{array}$ & -32.7 & $\begin{array}{l}\text { RFL was not } \\
\text { stable, re- } \\
\text { etched later }\end{array}$ \\
\hline $\begin{array}{l}\text { May 23 } \\
2014\end{array}$ & $\# 12$ & 45 & 250 & 4 & 10 & 0 & 3.65 & 6.78 & 4.5 & 6 & $\begin{array}{l}5.5 * 10- \\
6\end{array}$ & -42.7 & $\begin{array}{l}\text { sample looed } \\
\text { black }\end{array}$ \\
\hline $\begin{array}{l}\text { May } 23 \\
2014\end{array}$ & $\# 13$ & 50 & 256 & 5 & 10 & 0 & 3.65 & 6.78 & 4.5 & 6 & $\begin{array}{l}1.2 * 10- \\
5\end{array}$ & -34.1 & $\begin{array}{l}\text { very stable } \\
\text { conditions, } \\
\text { the sample } \\
\text { was black } \\
\text { with a small } \\
\text { grey }\end{array}$ \\
\hline $\begin{array}{l}\text { May 23 } \\
2014\end{array}$ & $\# 14$ & 21 & 251 & 5 & 10 & 0 & 3.65 & 6.78 & 4.5 & 6 & $\begin{array}{l}1.5 * 10- \\
5\end{array}$ & -44.3 & $\begin{array}{l}\text { The sample } \\
\text { was dark grey }\end{array}$ \\
\hline $\begin{array}{l}\text { May } 26 \\
2014\end{array}$ & $\# 14$ & 14 & 270 & 50 & 10 & 0 & 3.65 & 6.78 & 4.5 & 6 & $\begin{array}{l}1.5 * 10- \\
5\end{array}$ & -50.1 & $\begin{array}{l}\text { black and } \\
\text { brown, not } \\
\text { good as old } \\
\text { sample \#1, } \\
\text { after total } 35 \\
\text { min }\end{array}$ \\
\hline $\begin{array}{l}\text { May } 26 \\
2014\end{array}$ & $\# 15$ & 55 & 270 & 5 & 10 & 0 & 3.65 & 6.78 & 4.5 & 6 & $\begin{array}{l}2.2 * 10- \\
5\end{array}$ & -47.2 & $\begin{array}{l}\text { black and } \\
\text { grey, over } \\
\text { etched }\end{array}$ \\
\hline
\end{tabular}




\section{Appendix D: Fabrication Process Runsheet for Silicon Nanowires Solar Cells}

STARTING MATERIAL:

2 - 1-4 $\Omega \mathrm{cm} p<100>$ (pick lowest res. wafers) SW14-1,2

Record resistivity's . PV13-1 $=2$ ohm-cm, PV13-2 $=1.9$ ohm-cm,

PV13=2.16 ohm-cm, PV13-4 = 2.2 ohm-cm, PV13-5=2.2 ohm-cm

Brief Outline

1. SCREEN OXIDATION - 400nm

2. DEVICE WELL P.E. - RIE/BOE etch

3. PHOSPHORUS DIFFUSIONS

- 875deg C (standard - SW1,2),

4. NANOWIRES GROWTH IN UWO (Au/In)

5. BACK INDIUM REMOVAL - AQUA REGIN/HCL

6. BACK OXIDE REMOVAL - RIE

7. BACKSIDE 1\% HF SWAB TO HYDROPHOBIC

8. BACK METALLIZATION (0.5um Aluminum)

9. METAL SINTER 1-500 deg C N2

10. METAL LIFT-OFF LITHOGRAPHY

11. FRONT METALLIZATION (Titanium/Silver)

12. METAL LIFT-OFF

13. METAL SINTER $2-400 \mathrm{deg} \mathrm{H} 2$

14. ANTIREFLECTION COATING (spin on Titaniumsilica)

1. SCREEN OXIDATION

$400 \mathrm{~nm}$

Apex recipe\# Apex Run\#

a) RCA clean

b) Oxidation tube temperature $1000^{\circ} \mathrm{C}$

5 min preheat $\quad \mathrm{O}_{2}$

2" every 6 sec push $\quad \mathrm{O}_{2}$

10 min oxidation dry $\mathrm{O}_{2}$

65min wet oxidation $\quad \mathrm{O}_{2}+\mathrm{H}_{2}$

20 min oxidation $\quad \mathrm{O}_{2}+2 \% \mathrm{HCl}$

20 min anneal $\quad \mathrm{N}_{2}$

2 " every 15 sec pull $\quad \mathrm{N}_{2}$

10 min cool $\quad \mathrm{N}_{2}$

target thickness: $400 \mathrm{~nm}$

thickness measured on wafer: 
a) PlasmaPreen condition, (Plasmapreen condition not required if coming out of furnace tube) HMDS oven, apply S1811 photoresist, prebake, expose, develop, postbake, descum

(See Appendix A for standard photoresist procedure)

b) RIE etch: Fronts

etch 10 $\mathrm{min} @ \sim 40 \mathrm{~nm} / \mathrm{min}$ in 15\% O2 + 85\% $\mathrm{CHF}_{3}$

RF Power: - $350 \mathrm{~W}$ Pressure: 150 mTorr

Leave $\sim 70 \mathrm{~nm}$ of oxide in scribe channels

as measured with Nanospec.

c) etch in buffered HF @ $20^{\circ} \mathrm{C}$ until bare Si exposed

(scribe channels will go hydrophobic)

estimated etch time: 2.5 to $3 \mathrm{~min}$

actual etch times:

c) strip PR in PlasmaPreen

\section{PHOSPHORUS DIFFUSIONS}

Include one 4" $\mathrm{p}$ type $<100>, 1-5 \Omega \mathrm{cm}$ test wafer in each diffusion

a) RCA clean

b) First diffusion SW14-1, 2 and one 1-5 ohm-cm test wafer

Pre-deposition source: $\mathrm{POCl}_{3} @ 20^{\circ} \mathrm{C}$ tube temperature: $8^{\circ}{ }^{\circ} \mathrm{C}$

$$
\text { Apex recipe\#___ Apex Run\# }
$$

$\begin{array}{ll}5 \text { min preheat } & \mathrm{N}_{2}+\mathrm{O}_{2} \\ 30 \text { sec push } & \mathrm{N}_{2}+\mathrm{O}_{2} \\ 5 \text { min warm-up } & \mathrm{N}_{2}+\mathrm{O}_{2} \\ 5 \text { min dope } & \mathrm{N}_{2}+\mathrm{O}_{2}+\mathrm{N} \\ 10 \text { min flush/drive-in } & \mathrm{N}_{2}+\mathrm{O}_{2} \\ 30 \text { sec pull } & \mathrm{N}_{2}+\mathrm{O}_{2} \\ 10 \text { min cool } & \mathrm{N}_{2}\end{array}$

remove phosphosilicate glass: etch $40 \mathrm{sec}$ in $1 \% \mathrm{HF}$

measure sheet resistance test wafer

sheet resistance:

(expecting $\sim 59 \mathrm{ohm} / \mathrm{square}$, or $13 \mathrm{mv}$ on test wafer)

( 71ohm/square on PV wafers) 


\section{NANOWIRES GROWTH IN UWO}
a) HF buffer solution for $60 \mathrm{sec}$
b) Mount the substrate on a Mo sample holder using indium (In)
c) Removed any silicon oxide layer on the substrate by

a. Outgassing at $600{ }^{\circ} \mathrm{C}$ for $15 \mathrm{~min}$

b. $850{ }^{\circ} \mathrm{C}$ for $10 \mathrm{~min}$ at $4 \times 10^{-9} \mathrm{Torr}$

d) Load the sample in the UHV MBE

e) Au film of $1 \mathrm{~nm}$ thickness deposited at $4 \times 10^{15}$ atoms $\times \mathrm{cm}^{-2} \mathrm{~min}^{-1}$ growth rate

f) Anneal at $650{ }^{\circ} \mathrm{C}$ for $30 \mathrm{~min}$

g) Si deposited at $5 \times 10^{14}$ atmos $\times \mathrm{cm}^{-2} \mathrm{~min}^{-1}$ rate for $1 \times 10^{-8}$ Torr and $0.5,1,2,4$ hour

\section{BACK INDIUM REMOVAL}
a) Aqua Regia in beaks heated to $65^{\circ} \mathrm{C}$
b) Aqua Regia swab on substrate back surface
c) HCL swab on substrate back surface

\section{BACK OXIDE REMOVAL}

a) Flood coat front of wafers with PR to protect.

b) Dry etch in MRC RIE approx. 15min, O2/CHF3, 350W RF, 150mtorr.

c) remove PR with Acetone/IPA/Plasmapreen

\section{Backside 1\% HF swab to hydrophobic}




\section{BACK METALLIZATION}

Al e-beam evaporation on backs

target thickness: $0.5 \mu \mathrm{m}$

actual thickness:

9. METAL SINTER 1 -- NOTE in N2 RUN One wafer through as test to make sure looks ok. Tube temperature $500 \mathrm{C}$

5 min preheat $\mathbf{N}_{2}$

5 min tube purge $\quad \mathrm{N}_{2}$

20 second push $\quad \mathrm{N}_{2}$

10 min anneal $\mathrm{N}_{2}$

$20 \mathrm{sec}$ pull $\quad \mathrm{N}_{2}$

5 min cool $\quad \mathrm{N}_{2}$

10 min tube purge $\quad \mathrm{N}_{2}$

10. METAL LIFT-OFF LITHOGRAPHY Mask CU-

Dark Field

PlasmaPreen condition, HMDS oven

MicroChem LOR lift-off resist.

Flood coat and spin LOR 10B

Bake LOR layer on hotplate for 5 min @ 185-190 ${ }^{\circ} \mathrm{C}$

Flood coat and spin on S1811 positive PR@4000 RPM

Prebake on hot plate @ $100{ }^{\circ} \mathrm{C}$ for $1 \mathrm{~min}$.

Align wafer to mask

Expose _ seconds. Use standard exposure time for S1811

Develop the Bi layer in MF-321 developer for 45 seconds

Inspect at 100x (minimum) brightfield and darkfield.

DO NOT POSTBAKE

11. FRONT METALLIZATION - Balzers evaporator.

a) Oxygen plasma descum, 1 min. Maximum @ $100 \mathrm{~W}, 300 \mathrm{mT}$

b) Flash $1 \% \mathrm{HF}$ to remove native oxide

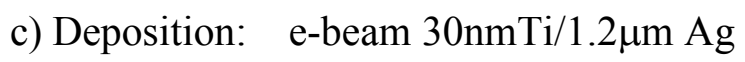


actual thickness:

12. METAL LIFT-OFF

Lift-off the layer in Microposit remover 1165 as follows:

Soak wafer in remover at $80^{\circ} \mathrm{C}$

Use two separate beakers, combined time may be $90 \mathrm{~min}$.

Rinse gently in running DI water for $15 \mathrm{~min}$.

Inspect for complete lift-off

If required, return the wafer to the second beaker of 1165 solution.

to remove residue.

Rinse wafer, then dry

NOTE: silver may look brown after this step but should clear up after sinter step.

\section{METAL SINTER 2}

Tube temperature $400 \mathrm{C}$

$\begin{array}{ll}5 \text { min preheat } \mathrm{N}_{2} & \\ 5 \text { min tube purge } & \mathrm{H}_{2} \\ 20 \text { second push } & \mathrm{H}_{2} \\ 10 \text { min anneal } & \mathrm{H}_{2} \\ 20 \text { sec pull } & \mathrm{H}_{2} \\ 5 \text { min cool } & \mathrm{H}_{2} \\ 10 \text { min tube purge } & \mathrm{N}_{2}\end{array}$

